\title{
Introduction to Geospatial Semantics and Technology Workshop Handbook
}

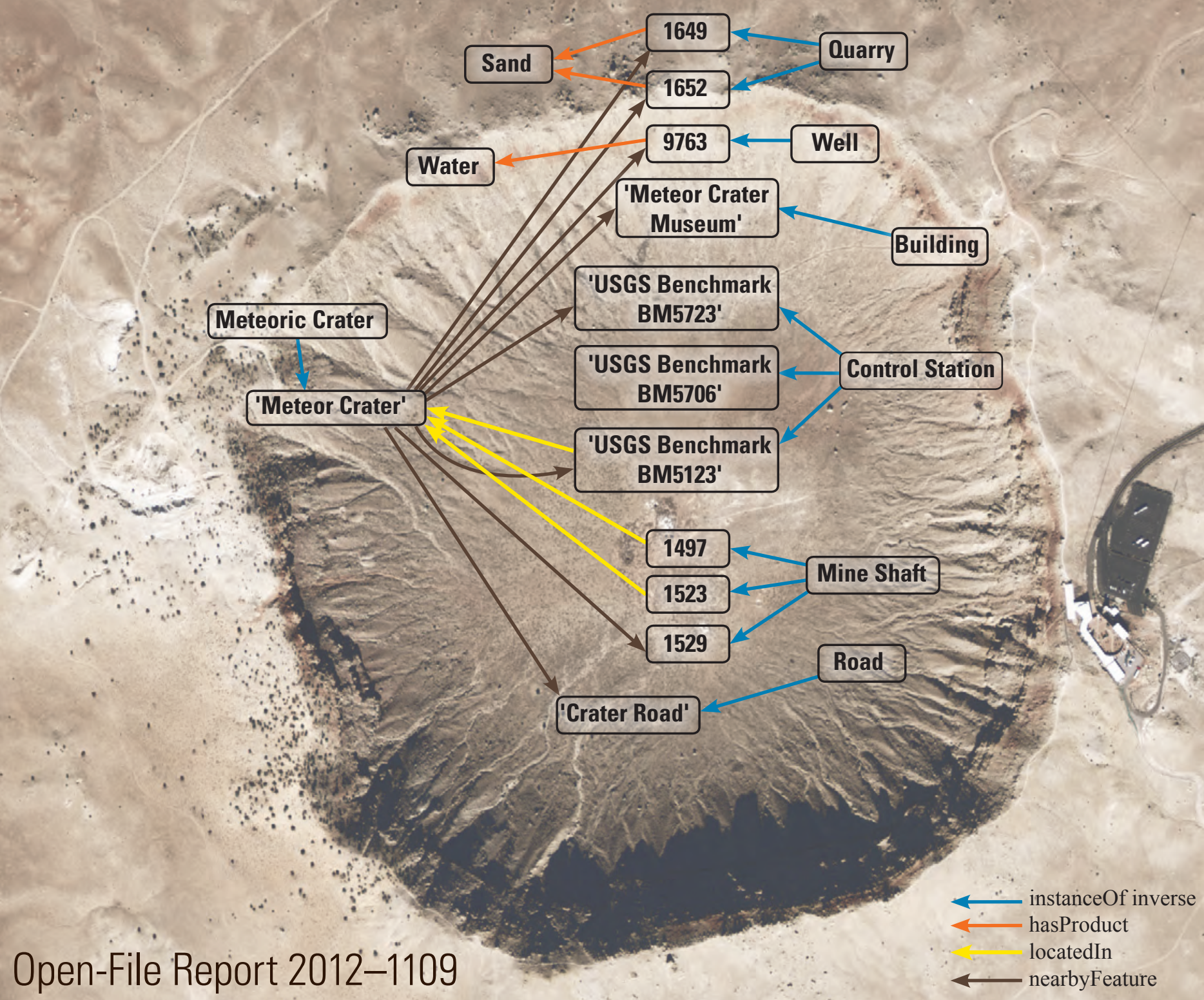

U.S. Department of the Interior

U.S. Geological Survey 
Cover photograph. Meteor Crater, Arizona (http://en.wikipedia.org/wiki/File:Meteor_Crater_-_Arizona.jpg). 


\section{Introduction to Geospatial Semantics and Technology Workshop Handbook}

Edited by Dalia E. Varanka

Open-File Report 2012-1109 


\title{
U.S. Department of the Interior \\ KEN SALAZAR, Secretary \\ U.S. Geological Survey \\ Marcia K. McNutt, Director
}

\author{
U.S. Geological Survey, Reston, Virginia: 2012
}

For more information on the USGS - the Federal source for science about the Earth, its natural and living resources, natural hazards, and the environment, visit http://www.usgs.gov or call 1-888-ASK-USGS.

For an overview of USGS information products, including maps, imagery, and publications, visit http://www.usgs.gov/pubprod

To order this and other USGS information products, visit http://store.usgs.gov

Any use of trade, product, or firm names is for descriptive purposes only and does not imply endorsement by the U.S. Government.

Although this report is in the public domain, permission must be secured from the individual copyright owners to reproduce any copyrighted materials contained within this report.

Suggested citation:

Varanka, D.E., ed., 2012, Introduction to geospatial semantics and technology workshop handbook: U.S. Geological Survey Open-File Report 2012-1109, 107 p. 


\section{Contents}

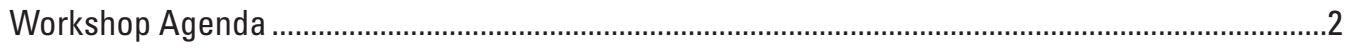

Workshop Summary and Slides .............................................................................................

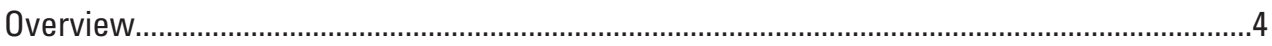

An Introduction to Semantic Web and Technology Concepts ..............................................

Accessing Linked Data Over the Internet ............................................................................3

The USGS Approach to the Geospatial Semantic Web.......................................................44

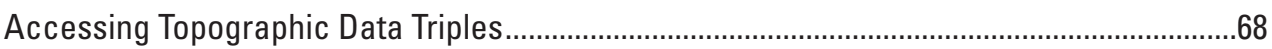

The SOCoP Open Ontology Repository (OOR) ................................................................92

Meteor Crater Ontology ..................................................................................................92

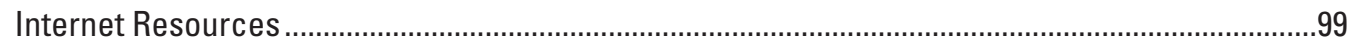

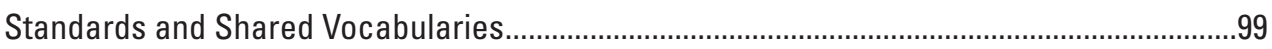

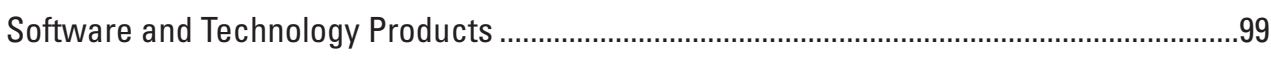

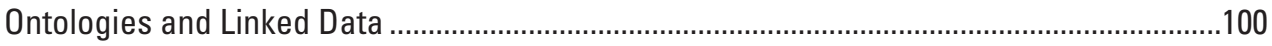

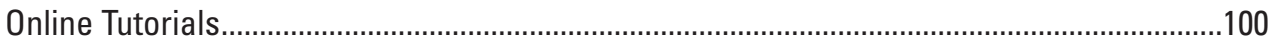

Ontology Communities, Professional Organizations, and Workshop Events .......................101

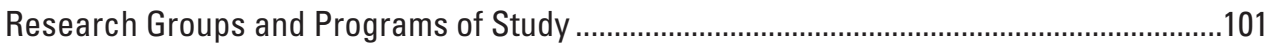

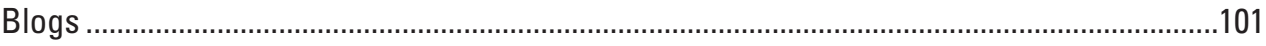

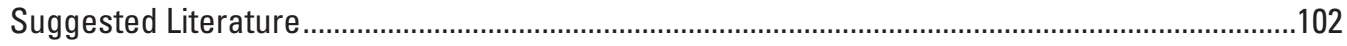

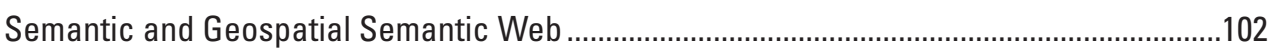

Geospatial Semantics and Ontology .................................................................................102

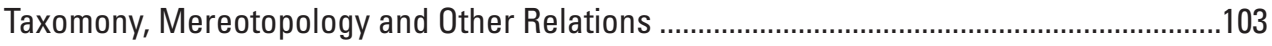

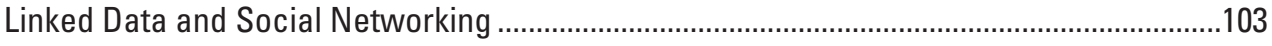

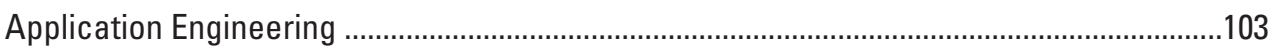

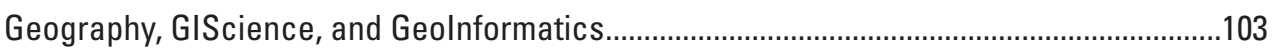

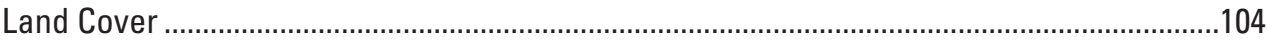

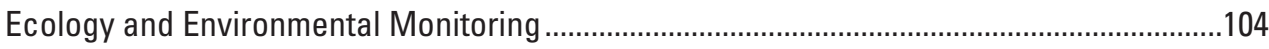

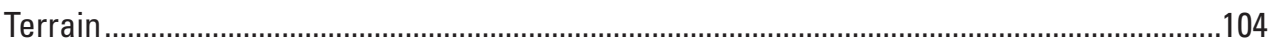

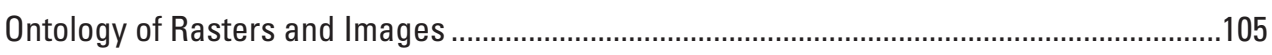

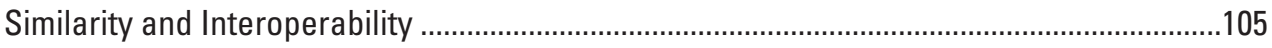

Logic and Knowledge Representation and Reasoning .....................................................105

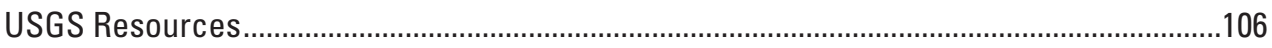

Edited Journal Issues and Proceedings from Scholarly Meetings .......................................106

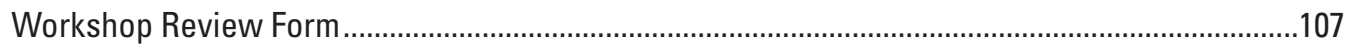





\title{
Introduction to Geospatial Semantics and Technology Workshop Handbook
}

\author{
University Consortium for Geographic Information Science \\ May 29, 2012 \\ Washington D.C.
}

\begin{abstract}
The workshop is a tutorial on introductory geospatial semantics with hands-on exercises using standard Web browsers. The workshop is divided into two sections, general semantics on the Web and specific examples of geospatial semantics using data from The National Map of the U.S. Geological Survey and the Open Ontology Repository. The general semantics section includes information and access to publicly available semantic archives. The specific session includes information on geospatial semantics with access to semantically enhanced data for hydrography, transportation, boundaries, and names. The Open Ontology Repository offers open-source ontologies for public use.
\end{abstract}

Dalia E. Varanka, Editor

USGS Center of Excellence for Geospatial Information Science (CEGIS)

http://cegis.usgs.gov/ontology.html

Spatial Ontology Community of Practice (SOCoP)

http://www.socop.org 


\section{Workshop Agenda}

12:00 - 1:00 pm Introduction to the workshop and to semantic technology concepts

Topics: Semantic Web standards and implementation examples, ontologies, and geospatial Semantic Web adaptations

1:00 - 2:00 pm Hands-on exercise: Accessing Linked Data over the Internet

Using the following URLs:

FOAF-A-MATIC

- http://ldodds.com/foaf/foaf-a-matic

\section{Tim Berners-Lee's FOAF page.}

- http://www.w3.org/People/Berners-Lee/

Start page for the GeoNames map

- http://www.geonames.org/6295630/

Download page for GeoNames

- http://www.geonames.org/ontology/documentation.html

Start page for the Faceted Search

- http://dbpedia.neofonie.de/browse

\section{URL for Virtuoso RDF Browser}

- http://dbpedia.org/fct/

DBpedia download page

- http://wiki.dbpedia.org/Downloads37 
2:00 - 3:00 pm USGS Approach to the Geospatial Semantic Web

Topics: Topographic data creation, ontology for The National Map, data retrieval, research needs in geospatial semantics

3:00 - 4:00 pm Hands-on exercises: Accessing topographic data triples

Executing queries with SPARQL Protocol and RDF Query Language (SPARQL) and GeoSPARQL, displaying results as URIs, and creating mapped output. URLS to use:

http://usgs-ybother.srv.mst.edu:8890/parliament/

http://usgs-ybother.srv.mst.edu/viz/

4:00 - 5:00 pm The Spatial Ontology Community of Practice (SOCoP) Open Ontology Repository

http://socop.oor.net/

Summary topics and wrap-up, discussion

Workshop Instructors: E. Lynn Usery, Dalia Varanka, Gary Berg-Cross

Workshop hands-on leads and support: David Mattli, Brian Collinge, Wayne Viers

Breaktimes will be scheduled during the workshop. 


\title{
Workshop Summary and Slides
}

\section{Overview}

\section{UCGIS/USGS \\ Geospatial Semantics Workshop}

\author{
Doubletree Hotel, Washington, D.C. \\ May 29, 2012
}

U.S. Department of the Interior

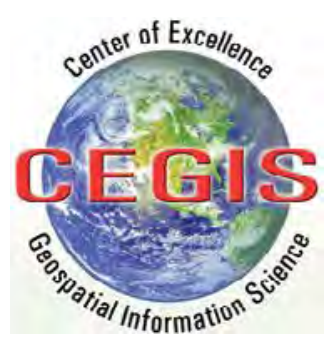

U.S. Geological Survey 


\section{Motivation - Why host a Workshop}

Geospatial semantics are future research and operational modes for GIS data

Lack of assimilation of semantics in GIScience community - e.g., Semantic Web appeared in 2001; not many GIScientists use it even now

Potential to expose USGS approach and data to public audience; outreach to gain feedback on USGS efforts

Basic tutorial on semantics is needed in GIScience community

\section{Introductory Level Tutorial}

The Workshop is an introductory tutorial on geospatial semantics

Introduces the Semantic Web and some general applications

Includes specific details of USGS data conversion, availability and access

This workshop assumes little prior knowledge, only an ability to work with computers and Web browsers 


\section{Goals of the Workshop}

Introduce semantic data on the Web

Introduce examples of Semantic Web applications

Introduce geospatial semantics

Provide USGS approach to semantics for geospatial data

Provide access to sample geospatial Resource Description Framework (RDF) data

\section{ॠUSGS}

\section{What you will learn}

Basic vocabulary and operation of Semantic Web

How geospatial data are structured as RDF

How to build new RDF data

How to convert existing legacy geospatial data

How to query RDF triplestores with SPARQL and GeoSPARQL 


\section{Some Topics Not Included}

Specific software packages

The SPARQL query language and syntax

Reasoning logic used in semantic applications

Specific ontological applications

\section{‡USGS}

\section{Instructors}

Dalia Varanka, Research Geographer, USGS

E. Lynn Usery, Research Geographer, USGS

David Mattli, Computer Scientist, USGS

Wayne Viers, Computer Scientist, USGS

Brian Collinge, Geographer, USGS

Gary Berg-Cross, Spatial Ontology Community of Practice 


\section{Workshop Organization}

Overview lecture on semantic concepts - Dalia Varanka

Hands-on exercise with Facebook, DBpedia, Geonames - Wayne Viers

Overview of USGS approach and geospatial semantics - E. Lynn Usery

Building ontology with Protégé - Brian Collinge

Hands-on exercises with USGS geospatial semantic data --- David Mattli

Open Ontology Repository - Gary Berg-Cross 


\title{
An Introduction to Semantic Web and Technology Concepts
}

The first section of the workshop introduces background concepts from geospatial semantics and ontology technology, including the triple data model, controlled vocabularies, standards, along with processes such as linking data for federated graphs, logical inference for automated knowledge creation, and information querying using SPARQL and GeoSPARQL protocols.

\section{Geospatial Semantics An Introduction to the Basics}

\author{
Dalia E. Varanka \\ Research Geographer \\ http://cegis.usgs.gov/ontology.html

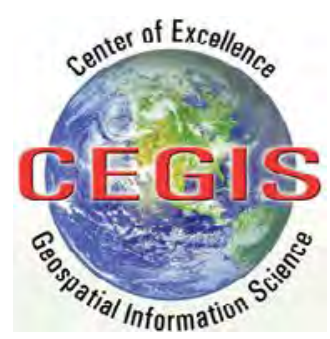




\section{Why the Semantic Web?}

Our world and particularly our cities form complex sociotechnical-natural systems, meta-systems, and systems of systems

More intelligence, instrumentation, application, integration

Big Data (volume, variety, velocity, value) driving new paradigms in science

\section{Semantics}

Semantics, the study of how humans derive meaning from representations, is a central approach for the design of new scales of systems and data Growing area of technical research since 2001

Rooted in artificial intelligence

Ontology: the structural framework for organizing meaningful information

Broadly based research field;

philosophy, linguistics, social science, engineering 


\section{Topics of this Introduction}

Semantic Web standards

Semantic technology implementation

Designing ontology patterns

Geosemantic adaptations

GeoSPARQL standard

\section{Internet Today}

Web page URLs and keywords pull out snippets of information; lack context

Linked data using tags self-driven interaction with the media

Data scarcity, generalization, and representation can all cause ambiguous information interpretations 


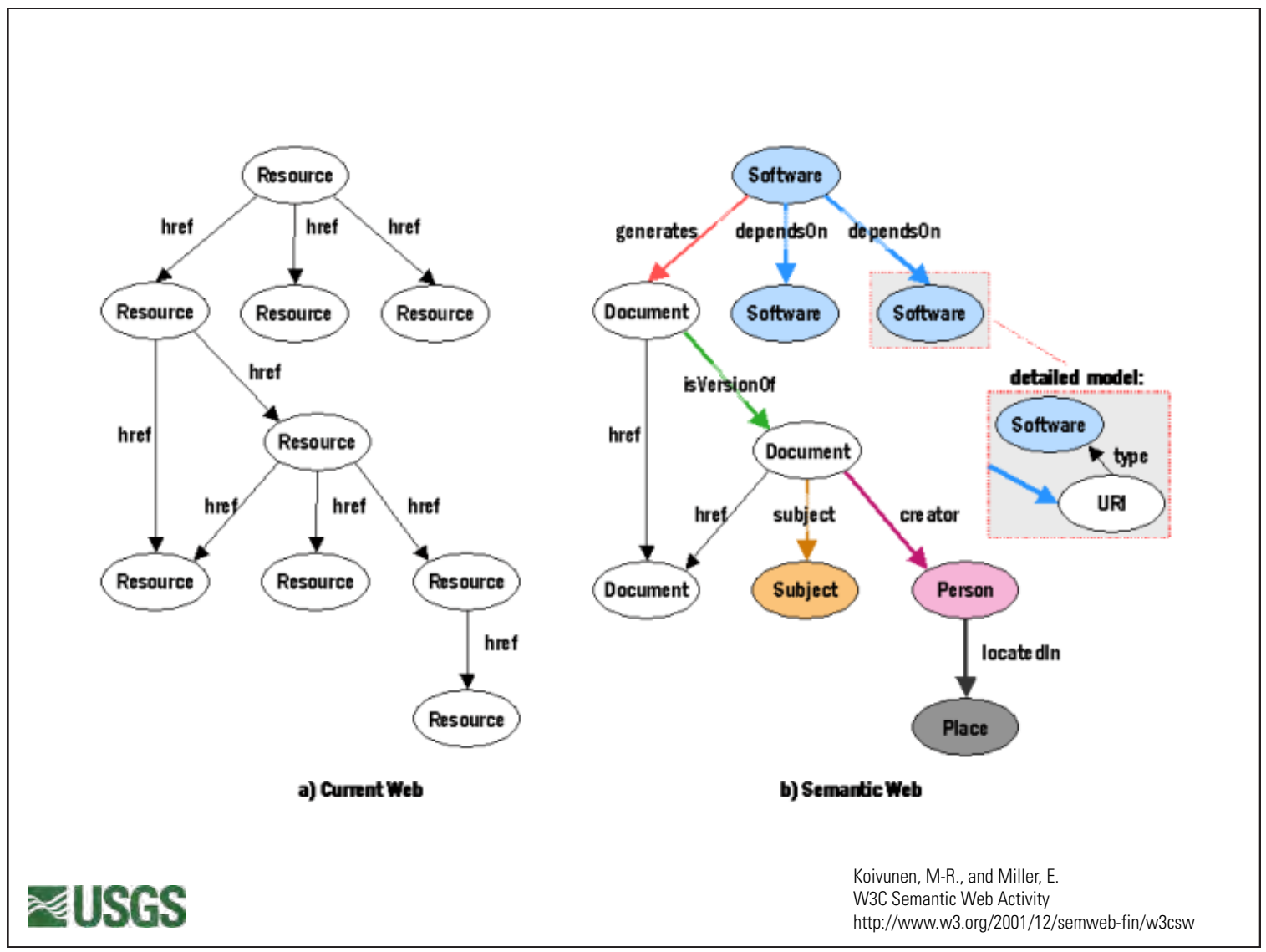

\section{Semantic System}

SPARQL Query

Endpoint

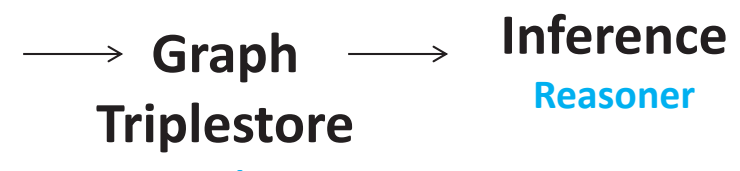

$$
\text { Java/Jena }
$$

URI

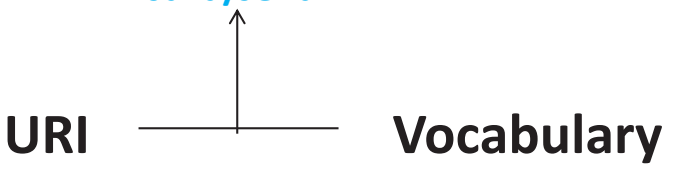

\section{Vocabulary}

Ontology Design Software

Text/Graphic 


\section{W3C Standards}

- Semantic specification of each datum

- Uniform Resource Identifiers (URI)

- Vocabulary

- Simple Knowledge Organization System (SKOS)

- Web Ontology Language (OWL)

- Linking data

- Resource Description Framework (RDF)

- Extensible Markup Language (XML)

- Query and Reasoning

- SPARQL Protocol And RDF Query Language (SPARQL)

- Rule Interchange Format (RIF)

ఇUSGS

\section{Semantic Specification}

Vocabulary

subject - predicate - object

Triple Resources

node - edge - node

Uniform Resource Identifier (URI):

http://cegis.usgs.gov/ontology/topovocab/1.0/terrain\#

Prefix: usgsTopo

usgs:_7945 rdf:type usgsTopo:Crater

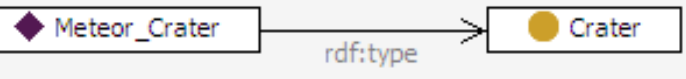




\section{Identifiers}

Uniform Resource Identifier (URI)

Uniform Resource Locator (URL)

Uniform Resource Name (URN)

Internationalized Resource Identifier (IRI)

URI can be

a lexical word

Random alpha-numeric unique identifier

\section{ఇUSGS}

\section{Examples of Standard and Controlled Vocabularies}

Philosophy terms (DOLCE)

Information content (NASA SWEET)

Metadata (Dublin Core Metadata Initiative)

Web Services (OGC Web Service Common)

Project terms (function, capability, role, purpose, objective, goal, etc.)

Task terms (map, compute, display) 


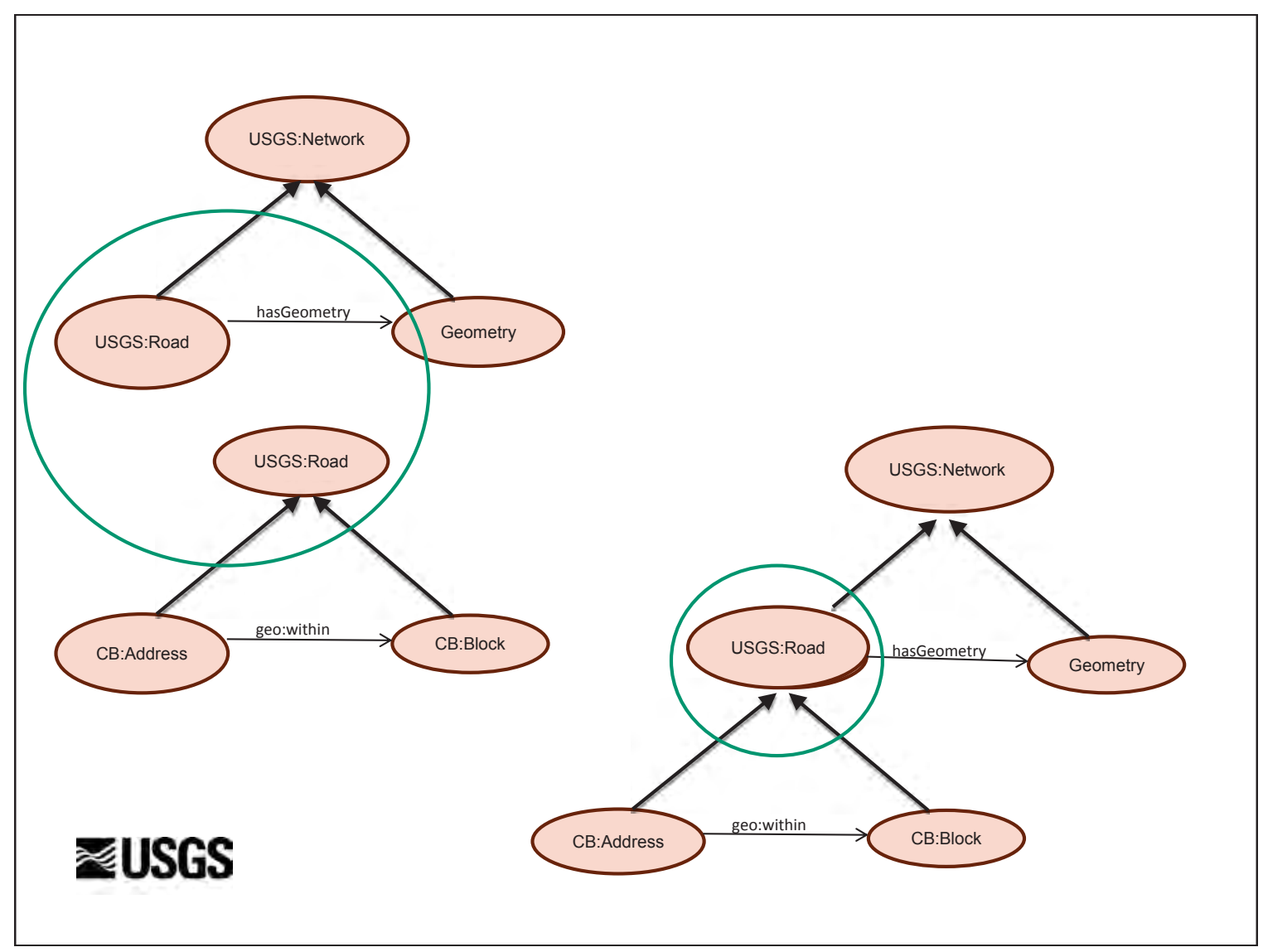

\section{Text Definition}

Census Bureau workstation html

\section{Vocabulary} is accessed through the Internet

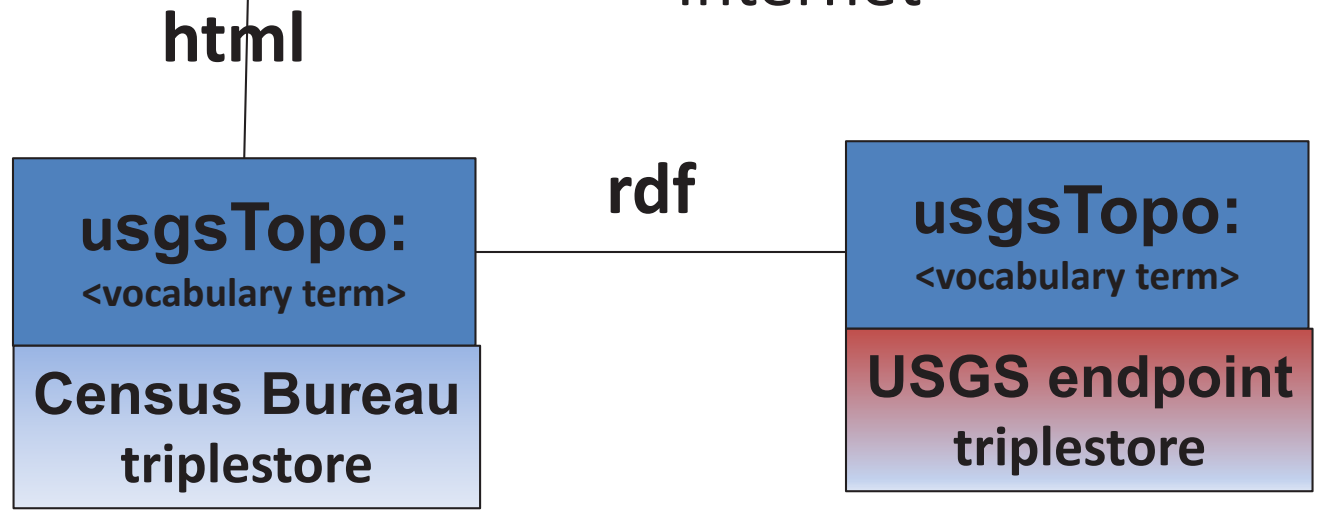




\section{Knowledge Representation and Reasoning (KRR)}

Simulates cognition

Language and interpretation

Reasoning forms

Logical

Experience and practice

Signs and semiotics

\section{Logic Rules as Predicates in OWL}

- ogc:intersects rdf:type owl:SymmetricProperty . usgsTopo:RoadA ogc:intersects usgsTopo:Road .

- usgs:woodland owl:equivalentClass usgsTopo:woodedArea 


\section{Inference and Logic Rules}

Information can be inferred based on logical rules

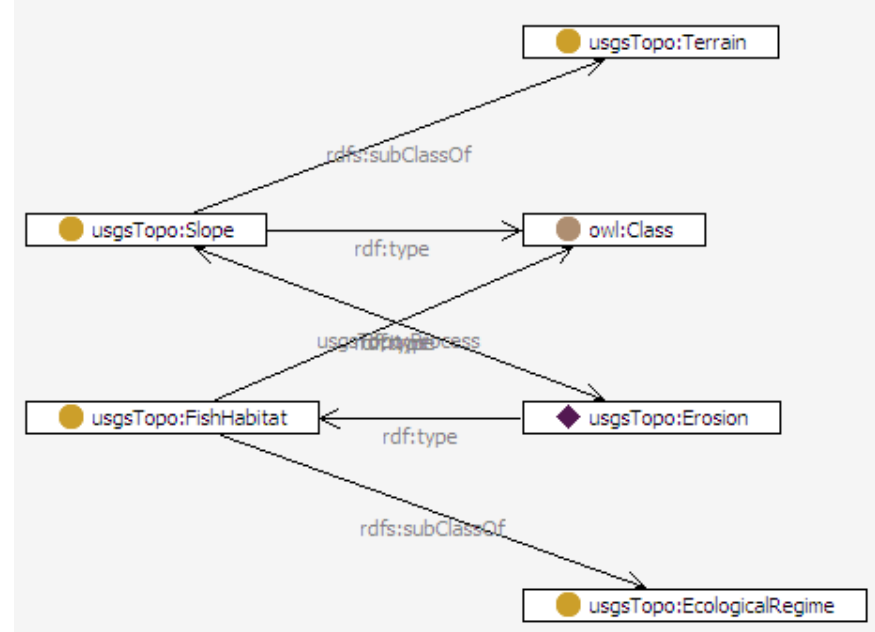

₹USGS

\section{Inference Properties}

- RDF Schema (RDFS) inference

- Selected elements of OWL

- equivalent classes and properties

- inverse properties

- symmetric properties

- functional properties

- inverse functional properties

- transitive properties 


\section{SPARQL}

First part defines prefixes of URI namespaces

SELECT clause identifies the variables to be returned

\section{SELECT ?feature}

WHERE clause gives the triple patterns defining a basic graph pattern

$$
\text { WHERE \{?feature gnis:name ‘\#name\#’\} }
$$

\section{Solution and Results}

Graph pattern given in the WHERE clause is matched against the triple store

An exact match to the pattern is required

Matching variables bind together to form the query solution

The values of the variables are the query results 


\section{Implementations}

\section{Converting Table Information to Triples}

Rows are subjects, columns are predicates, and cells are objects

\begin{tabular}{|l|l|}
\hline Feature & Process \\
\hline Mountain Range & Tectonics \\
\hline Delta & Deposition \\
\hline Peak & Erosion \\
\hline Volcano & Tectonics \\
\hline Arch & Erosion \\
\hline
\end{tabular}

Mountain Range hasProcess Tectonics Delta hasProcess Deposition

Peak hasProcess Erosion

Volcano hasProcess Tectonics

Arch hasProcess Erosion 


\section{Ontology to Database Interface}

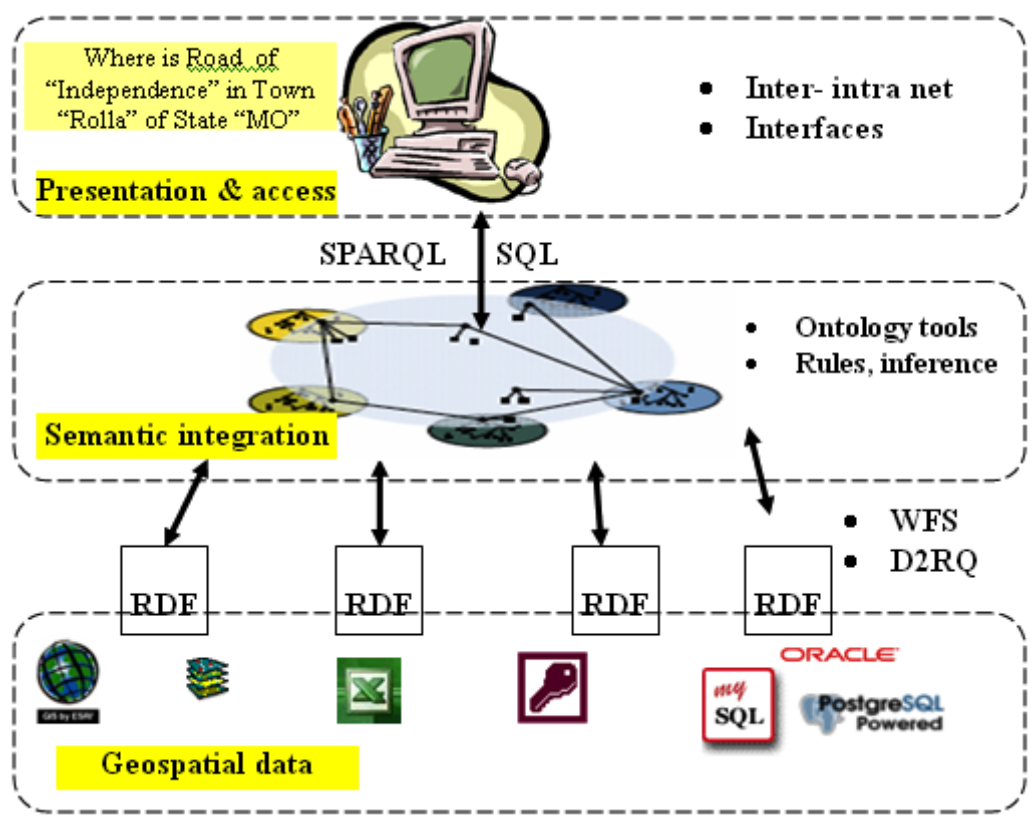

\section{Challenges}

Legacy resources are ambiguous

Triple stores quickly become volumetrically large Is scaling up to the entire web possible? Input data must be formatted -

Standardization vs. bottom-up information Concept commitments vs. multiple perspectives 


\section{Limited Applications}

Semantic technology to resolve a specific problem

Metadata indexing for discovery, access, and management

Oak Ridge National Laboratory MERCURY

Vocabulary sharing

USGS Integrated Taxonomic Information System

(ITIS) resolves Life Science ID (LSID) formats to RDF

ఇUSGS

\section{Hybrid Applications}

Content management

Drupal

Social networking data

Facebook Open Graph

Statistics

Gene Ontology (GO)

Mathematical functions

A large body of literature 


\section{Ontology Design Pattern}

An ontology pattern is

a modeling solution for a recurrent ontology designing problem

a template that represents the necessary and sufficient conditions as a base for specific design solutions

a set of "prototypical" ontology entities that constitute the "abstract form" of a pattern or schema

\section{ODP for Place}

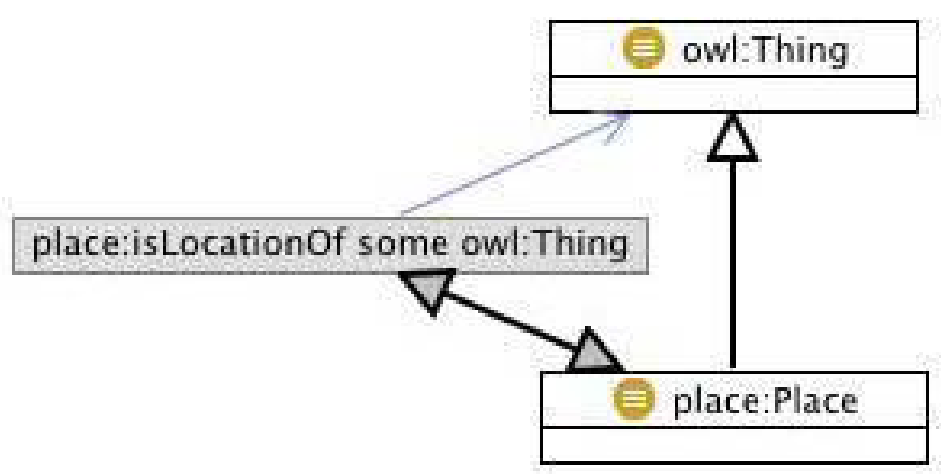




\title{
Method-Concept Mapping
}

\author{
Narrative and vocabulary are analyzed and \\ converted to logical diagrams and computer \\ applications
}

ఇUSGS

\section{Begin With Reuseable Vocabularies}

@prefix geonames: <http://www.geonames.org/ontology\#>.

@prefix rdfs: <http://www.w3.org/2000/01/rdf-schema\#> .

@ prefix rdf: <http://www.w3.org/1999/02/22-rdf-syntax-ns\#> .

@prefix owl: <http://www.w3.org/2002/07/owl\#>.

@ prefix dcterms: <http://purl.org/dc/terms/>

@prefix dbpedia: <http://dbpedia.org/ontology/>

@prefix geo: <http://www.opengis.net/ont/OGC-GeoSPARQL/1.0/>

@prefix usgsTopo: <http://cegis.usgs.gov/TopoVocab/1.0/Terrain\#> .

@prefix usgs: <http://cegis.usgs.gov/ontology/instances\#> . 


\section{Construct a Taxonomy}

Semantics of the relation

is-a, class inclusion, or subsumption

Different kinds of relations

Generalization, subsets, specialization

Structural similarities between descriptions

Semantic similarity measurement

Geosemantic Adaptations 


\section{Topographic Data Semantics}

Wide appeal for participatory mapping

Basic categories

Category theory

A physical 'real world'

Helps disambiguate cognitive/cultural differences

\section{Geospatial RDF Standards}

W3C:

Basic Geo Vocabulary (2003)

Location points combined with other ontology

GeoRSS (2006)

Location points with Really Simple Syndication

GeoOWL ontology (2005)

Expanded the geosemantic vocabulary to include toponyms, spatial relations, coordinate reference systems, metadata, and web services 


\section{Geospatial Features as Triples}

<http://cegis.usgs.gov/ontology/instances> a owl:ontology

usgs:_7945 a usgsTopo:crater;

a geo:Feature ;

geo:hasGeometry usgs:_7945geo ;

geoname:name "Meteor Crater"

rdfs:comment "A meteor crater";

usgsTopo:hasBenchmark usgs:_5723 ;

usgsTopo:hasBenchmark usgs:_e5706 ;

dcterms:identifier "7945" ;

dcterms:description "Circular-shaped depression on the surface of the land caused by the impact of a meteorite"

usgs:_7945geoa geo:Geometry ;

usgsTopo:hasShape "circular" ;

usgsTopo:width "0.2km" ;

usgsTopo:innerDiameter "833m" ;

usgsTopo:outerDiameter "1250m" .

usgsTopo:hasUTM "E 497959.94m N 3876020.68m Zone 12";

usgsTopo:hasPLSS "T 19 N, R 12 1/2 E, Section 13 and 24";

usgsTopo:hasMBR "Max E 489536.79m Min E 497317.62m Max N 3876632.29m Min N 3875479.58m";

dbpedia:MaximumElevation "5723ft";

dbpedia:MinimumElevation "5123ft";

dbpedia:MaximumDepth "600ft" .

\section{Topological Relations}

\section{Relations based on interior, boundary, and exterior contact between two features}

\section{OGC Simple Feature}

Terms

-Equals

-Disjoint

-Intersects

-Touches

-Within

-Contains

- Overlaps

- Crosses

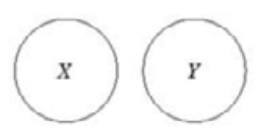

$X$ DC $Y$

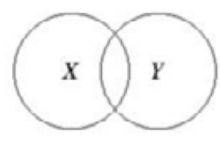

$X$ PO $Y$

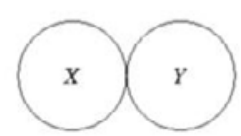

$X$ EC $Y$

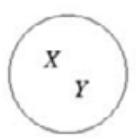

$X$ EQ $Y$

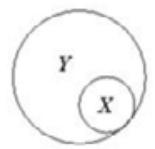

$X$ TPP $Y$

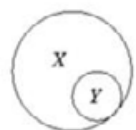

$X$ TPPi $Y$

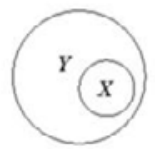

$X$ NTPP $Y$

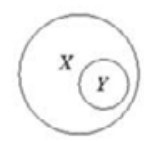

$X$ NTPPi $Y$ 


\section{GIS vs. Geosemantic Topology}

- GIS topology is not converted

- Topology is calculated with

- Well Known Text (WKT)

- Geography Markup Language (GML)

\section{‡USGS}

\section{GeoSPARQL Ontology}

geo:SpatialObject

geo:Feature geo:hasGeometry geo:Geometry

geo:defaultGeometry

geo:asWKT / sf:wktLiteral for values

geo:asGML / gml:gmlLiteral for values 


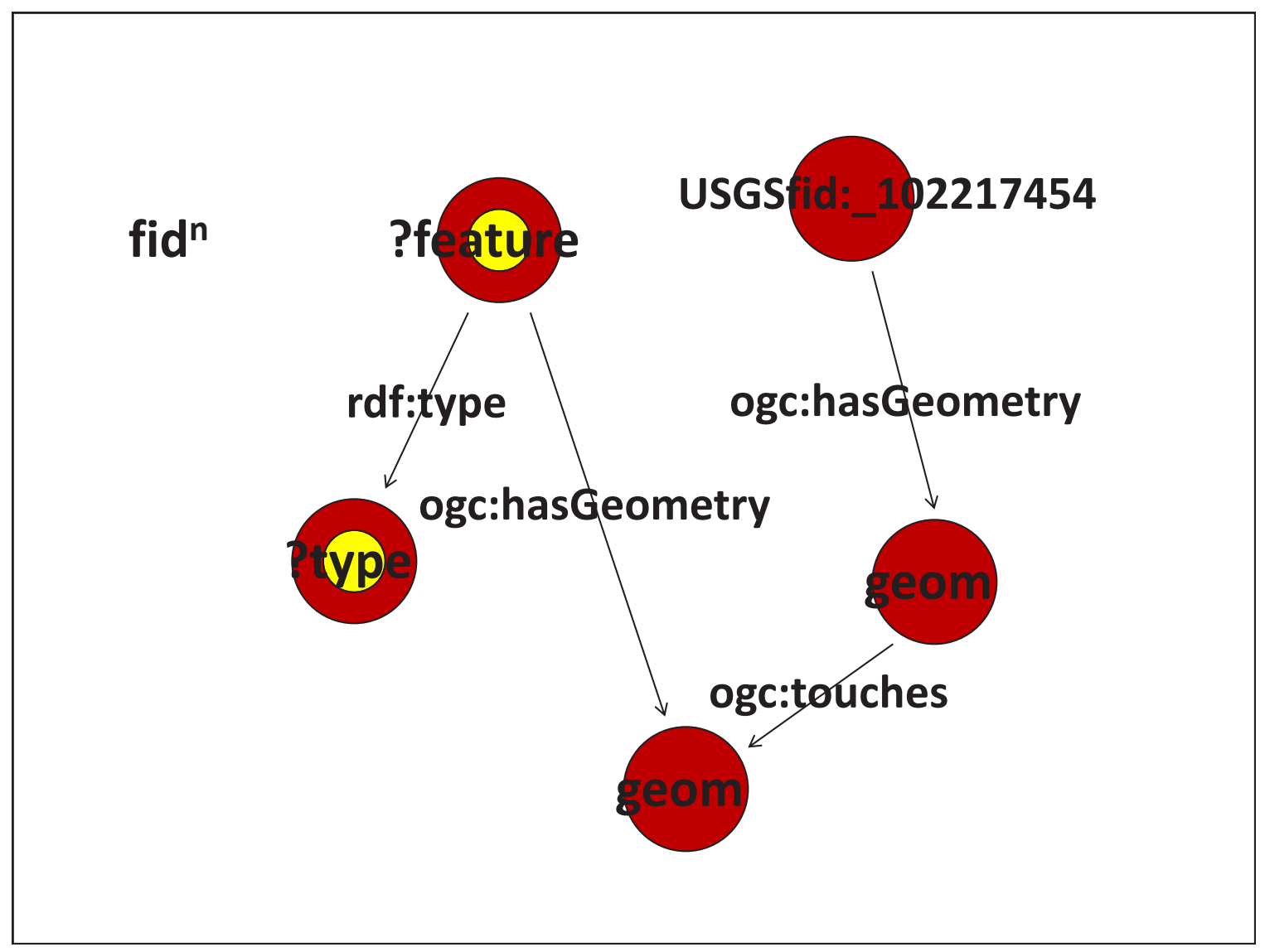

\section{GeoSPARQL Filter Functions}

Operator functions take multiple geometries as predicates and produce either a new geometry or another datatype as a result

- ogcf:intersection returns a geometry produced by the spatial intersection of two geometries

- ogcf:distance produces xsd:double

Boolean topological tests of geometries 


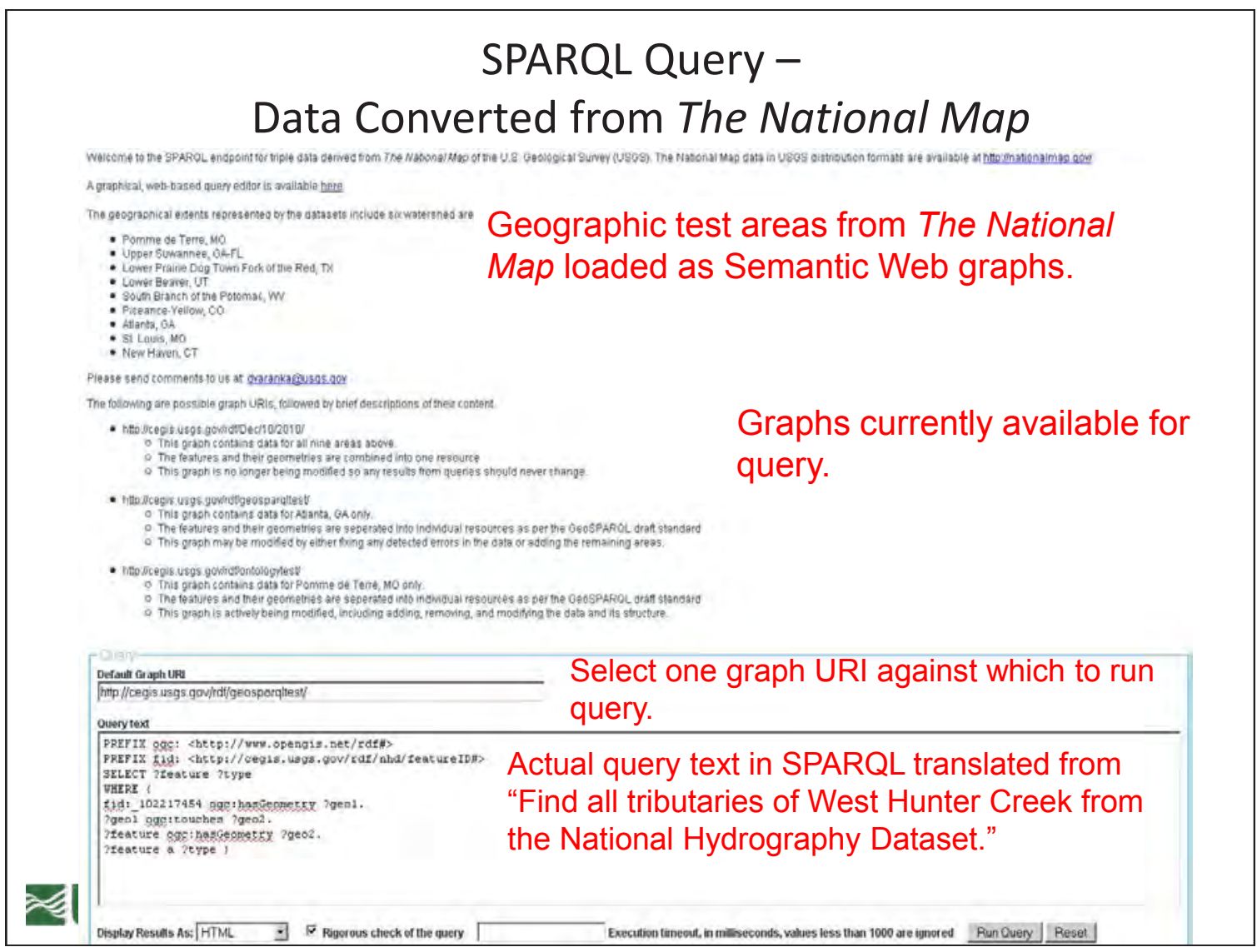

\section{SPARQL Query Results with Uniform Resource Identifiers (URI)}

\begin{tabular}{|c|c|}
\hline feature & type \\
\hline http $/ /$ cegis.usgs.gov/rdf/nhd/featureID\#_102216358 & http $/ /$ cegis.usgs.gov/rdf/nhd\#Stream_River \\
\hline http://cegis.usgs.gov/rdf/nhd/featureID\#_102216276 & http $/ /$ cegis.usgs.gov/rdf/nhd\#Stream_River \\
\hline http://cegis.usgs.gov/rdf/nhd/featureID\#_102216320 & http $/ /$ cegis.usgs.gov/rdf/nhd\#Stream_River \\
\hline http://cegis.usgs.gov/rdf/nhd/featureID\#_102216340 & http $/ /$ cegis.usgs.gov/rdf/nhd\#Stream_River \\
\hline http $/ /$ cegis.usgs.gov/rdf/nhd/featureID\#_102216448 & http $/ /$ cegis.usgs.gov/rdf/nhd\#Stream_River \\
\hline http://cegis.usgs.gov/rdf/nhd/featureID\#_102216432 & http://cegis.usgs.gov/rdf/nhd\#Stream_River \\
\hline http://cegis.usgs.gov/rdf/nhd/featureID\#_102216442 & http $/ /$ cegis.usgs.gov/rdf/nhd\#Stream_River \\
\hline http://cegis.usgs.gov/rdf/nhd/featureID\#_102216272 & http $/ /$ cegis.usgs.gov/rdf/nhd\#Stream_River \\
\hline http//cegis.usgs.gov/rdf/nhd/featureID\#_102216308 & http $/ /$ cegis.usgs.gov/rdf/nhd\#Stream_River \\
\hline http://cegis.usgs.gov/rdf/nhd/featureID\#_102216274 & http $/ /$ cegis.usgs.gov/rdf/nhd\#Stream_River \\
\hline http//cegis.usgs.gov/rdf/nhd/featureID\#_102216382 & http $/ /$ cegis.usgs.gov/rdf/nhd\#Stream_River \\
\hline http://cegis.usgs.gov/rdf/nhd/featureID\#_102216400 & http $/ /$ cegis.usgs.gov/rdf/nhd\#Stream_River \\
\hline
\end{tabular}




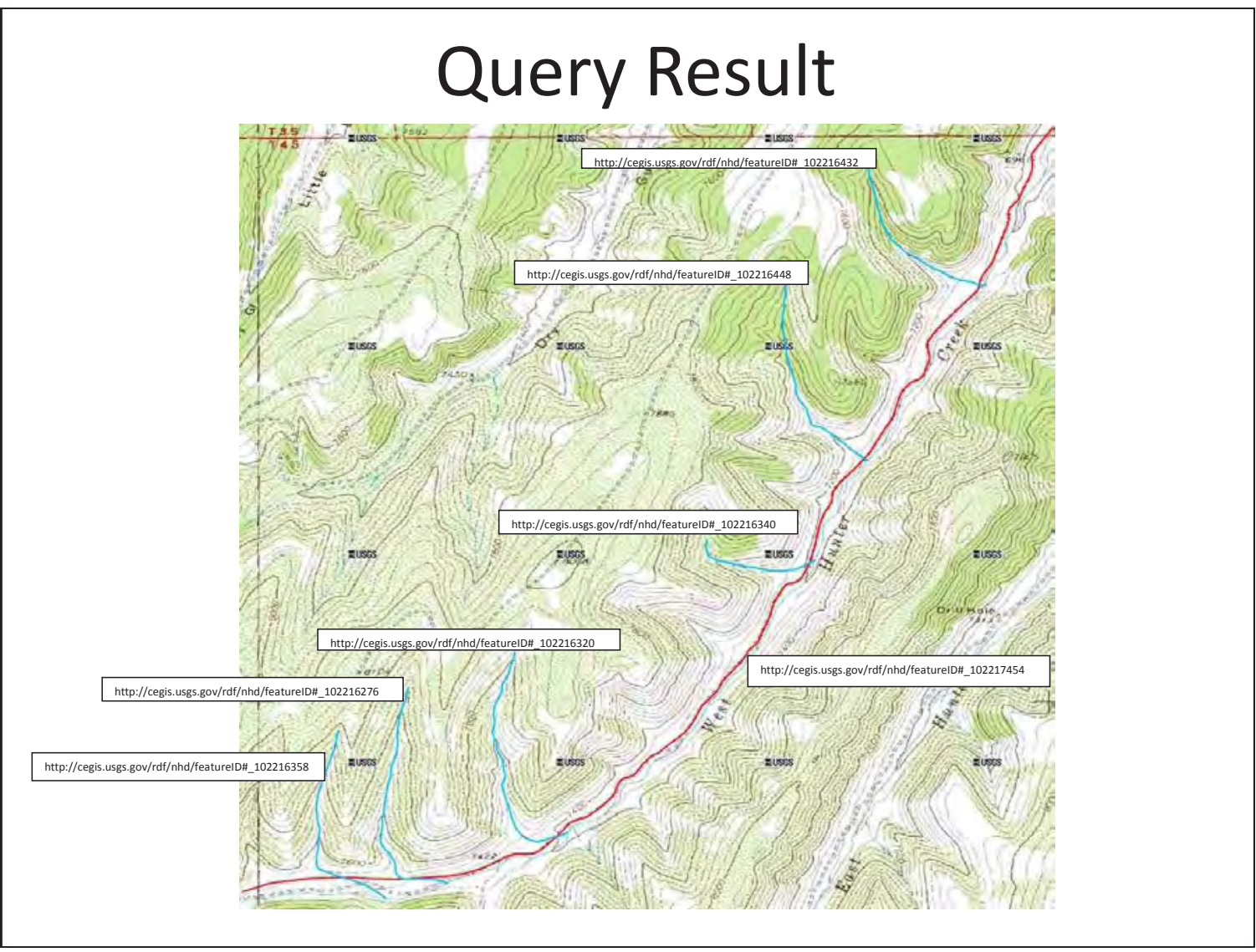

\section{Summary: Ontology is Expressed at Multiple Levels}

Formalized logical structures of knowledge Cognitive world views

Narratives and vocabularies of commonly shared words and meanings

Discourses about things

Applied representations based on concepts and their relations to each other

A map is an ontology

\section{₹USGS}




\section{On-line Demonstrations of Semantic Web sites}

- Semantic Web ontology example

- File formats handle triples in various ways

- Geonames.org toponym ontology

- Basic Geo point coordinates

- DBpedia

- At the center of linkeddata.org

\section{‡USGS}

\section{The USGS Project}

Data Conversion from The National Map

Reuseable ODP for base data

Graphical output for topographic mapping 


\section{Publicly Contributed Approaches}

- GeoVoCamp

- Community-based approaches to controlled vocabulary development

- Communities of Practice

- Spatial Ontology Community of Practice (SOCoP)

- Open Ontology Repository Initiative

§USGS 


\title{
Accessing Linked Data Over the Internet
}

Concepts introduced in the first hour are illustrated with examples using interactive data search, access, and download sites on the Internet. Sites to be visited are the Friend-of-a-Friend ontology, Geonames.org, and DBpedia, the Semantic Web version of Wikipedia.

\section{Applications of Semantic Technology}

\author{
Wayne Viers
}

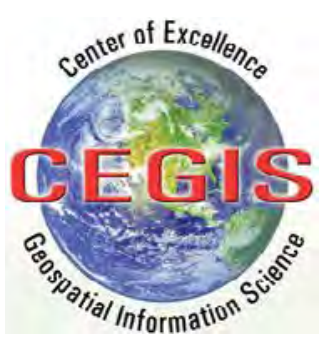




\section{Outline}

Three organizations that effectively use RDF FOAF: Example of RDF/XML generation Geonames: Example of Semantic Web DBpedia: Example of semantic querying

\section{Friend of a Friend}

Goal: Create a web of machine readable pages describing people and the links between them An ontology designed to allow portability of information between Web sites 


\section{Exercise}

http://www.Idodds.com/foaf/foaf-a-matic

We will use FOAF-a-Matic to show how one can easily create a FOAF RDF page

The triples are generated in RDF/XML, which is a syntax for representing RDF in XML In this exercise, all triples have a common subject (you!)

This RDF/XML file can be added to your website to contribute to the Semantic Web

Example FOAF Page

A good example of a FOAF page is Tim Berners-Lee's

Web page as seen in your browser

http://www.w3.org/People/Berners-Lee/

Embedded RDF/XML (Can be viewed as XML or using your browser's View Source option.) http://www.w3.org/People/BernersLee/card.rdf 


\section{Web page as seen in your browser}

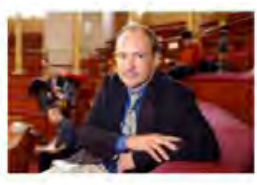

Short bio

Before you malt me

Address

Talls, articles etc

Speaking engagements

Press interviews

Tim Berners-Lee to

See also

Longer Bio

Talks

Design issues: web architecture

kids Questions

Weaving the Web - the book

Bio

A graduate of Oxford University. Tim Berners Lee invented the World Wide Web, an internet-based hypermedia initiative for slobal information sharing while at CERN, the European Particle Physics Laboratory, in 1989. He wrote the first web ctient and server in 1990 . His specifications of URL, HTTP and HTML were refined as Web technology spread.

He is the 3 Com Founders Professor of Engineering in the School of Engineering with a joint appointment in the Department of Electrical Engineering and Computer Sclence at the Laboratory for Computer Sclence and Artificlal intelligence ( CSAiL) at the Massachusetts Institute of Technology (MiT) where he also heads the Decentralized information Group (DIG). He is also a Professor in the Electronics and Computer Science Department at the University of Southampton, UK.

He is the Director of the World Wide Web Consortium (W3C), a web standards organization founded in 1994 which develops interoperable technologies (specifications, guldelines, software, and tools) to lead the Web to its full potential. He was a Director of the Web Sclence Trust (WST) launched in 2009 to promote research and education in Web Sclence, the multidisciplinary study of humanity connected by technology.

Tim is a Director of the World Wide Web Foundation, launched in 2009 to coordinate efforts to further the potential of the Web to benefit humanity.

He has promoted open government data globally and is a member of the UK's Transparency Board.

In 2001 he became a Fellow of the Royal Soclety. He has been the reciplent of several international awards including the Japan Prize, the Prince of Asturlas Foundation Prize, the Millennlum Technology Prize and Germany's Die Quadriga award. in 2004 he was knighted by H.M. Queen Elizabeth and in 2007 he was awarded the Order of Merit. in 2009 he was elected a foreign associate of the National Academy of Sciences. He is the author of "Weaving the Web".

(Longer blo)

Before you mail me

\section{Embedded RDF/XML}

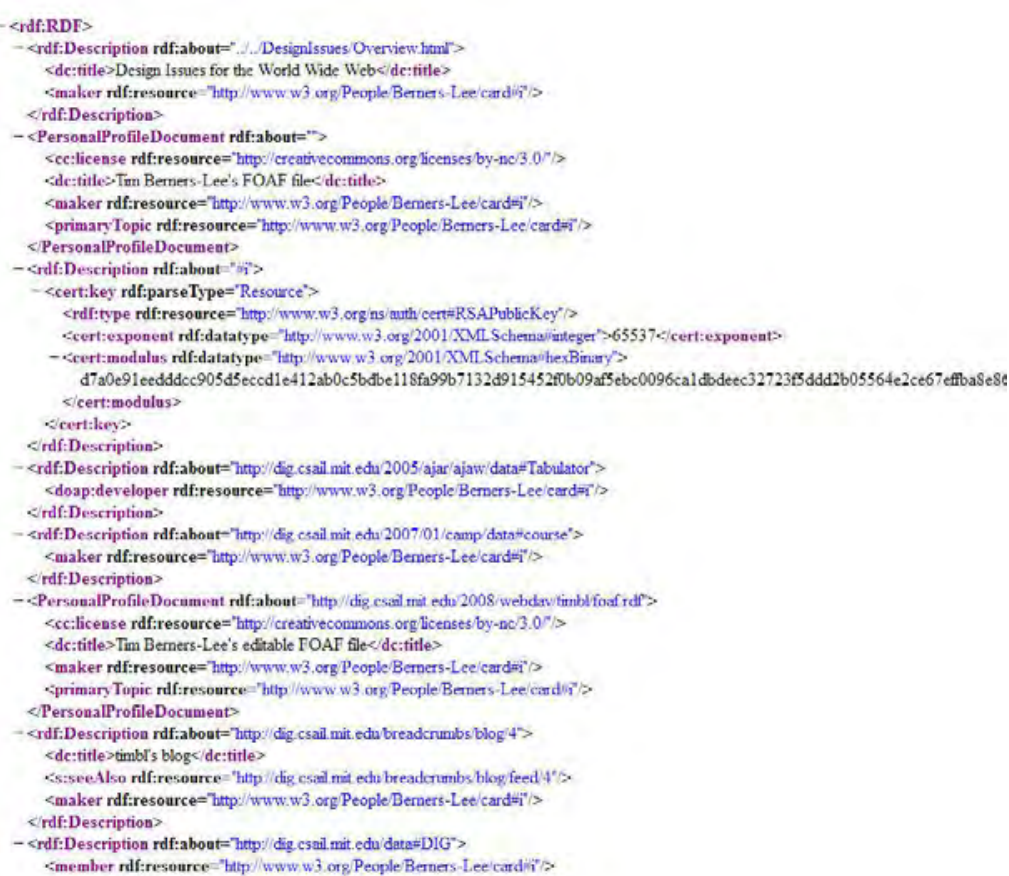




\section{Geonames}

Contains more than 10 million geographical names and more than 8 million unique features

More than 6.2 million Geonames toponyms have a unique URL

Has a map service that can display the semantic information of displayed features

\section{ॠUSGS}

\section{Exercise}

We will traverse Geonames semantic web to find the Washington Monument's Wikipedia page starting from the National Mall's semantic page.

To do this we will be using an online application called XMLGrid 


\section{xmlgrid.net}

http://xmlgrid.net/

We will use the sites XML Viewer to look at

$\mathrm{RDF} / \mathrm{XML}$ embedded in the Geonames map

http://www.w3.org/People/Berners-

Lee/card.rdf

Click the "By URL" box

Paste this link into the text box

Click submit, then "TextView"

₹USGS

\section{Geonames Map}

http://www.geonames.org/6295630/

Search for "National Mall" in the search bar in the top right

Click the purple "S" symbol next to the National Mall in the list below the map Adjust your zoom level so that you have a good view of the National Mall and the Washington Monument. 


\section{Solution}

Click the National Mall marker on the map then click "semantic web rdf"

Copy and paste the "nearbyFeatures" URL's into xmlgrid as shown previously, until you find the Washington Monument's RDF page.

Finally find the link to the Wikipedia article (making sure you select the one with en.wikipedia for English)

\section{Download Links}

http://www.geonames.org/ontology/document ation.html

Can download the entire RDF dataset but the file is very large (2.19 Gigabytes) 


\section{DBpedia}

DBpedia is a community effort to extract info from Wikipedia and make sophisticated queries against it.

Dataset contains 3.64 million "things" and over 1 billion triples

Triples built from info boxes in Wikipedia

\section{Faceted Search}

DBpedia uses the semantic information from Wikipedia pages to perform faceted searches

A facet is an aspect of a feature (elevation for example)

Very useful for finding sets of data with specific qualities

http://dbpedia.neofonie.de/browse 


\section{Exercise}

We will perform a spatial query using the faceted search to find...

Cities

With population between 40,000 and 65,000

And an elevation of 330 meters to 453 meters

\section{₹USGS}

\section{Solution}

Since city is not one of the visible facets, type city into the item type field on the left

Type 330 and 453 into the appropriate fields under the elevation facet

Finally enter 40000 and 65000 into the fields under the population total facet 


\section{RDF Web Browser}

\section{OpenLink Virtuoso built-in Faceted Browser Virtuoso is a database engine made by OpenLink Software}

The Virtuoso data base contains all the triples the browser searches over

DBpedia's triples can also be displayed in a browser in addition to being queried http://dbpedia.org/fct/

\section{₹USGS}

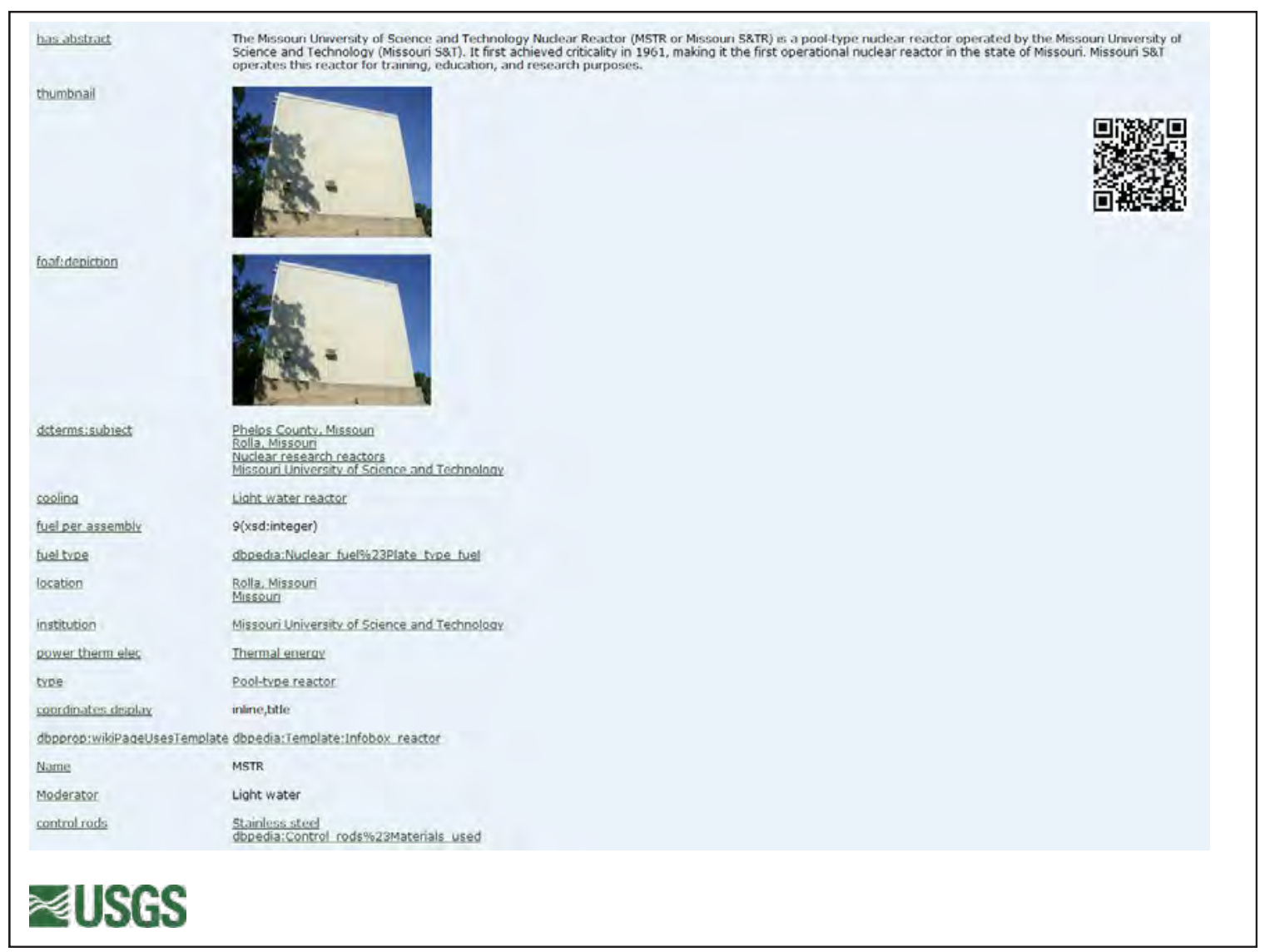




\section{Download Links}

http://wiki.dbpedia.org/Downloads37

Can download the DBpedia ontology in the OWL format (Web Ontology Language) Can be viewed in an ontology editor like Protégé. 


\section{The USGS Approach to the Geospatial Semantic Web}

In the second half of the workshop, the motivation for the USGS geospatial applications, including data integration and information retrieval lead to a discussion of approaches for enabling The National Map topographic data on the geospatial semantic web. A discussion of raster-based data semantics will be included.

\section{Geospatial Semantic Technology A Case Study with USGS Data}

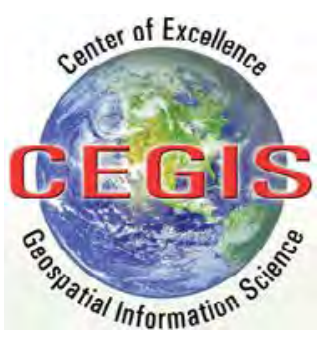




\section{Outline}

USGS Data Issues and Examples

The National Map

Semantics as a Solution

Building Semantics for Geospatial Data

Creating New Data with Semantics

Converting Legacy Data

Data Archive and Access

USGS Sample Data as RDF

Ontology for The National Map

Taxonomy of Domains

Topographic Vocabulary

Querying USGS Sample Data with SPARQL and GeoSPARQL

Using USGS Data with Other Data Sources

Linked Open Data

USGS Research Needs in Geosemantics

Future of Semantic Data at USGS

\section{‡USGS}

\section{USGS Data Issues}

Volume - multiple nationwide datasets at high resolution

Structure - variety of structures, vector and raster, many different formats

Semantics - various attribution and relation schemes, some feature-based, some layers

Integration of multiple datasets - for maximum utility all datasets should be able to be integrated to produce new data and information

Integration with data from users - users require the ability to add their data to USGS base datasets 


\section{Examples of USGS Datasets}

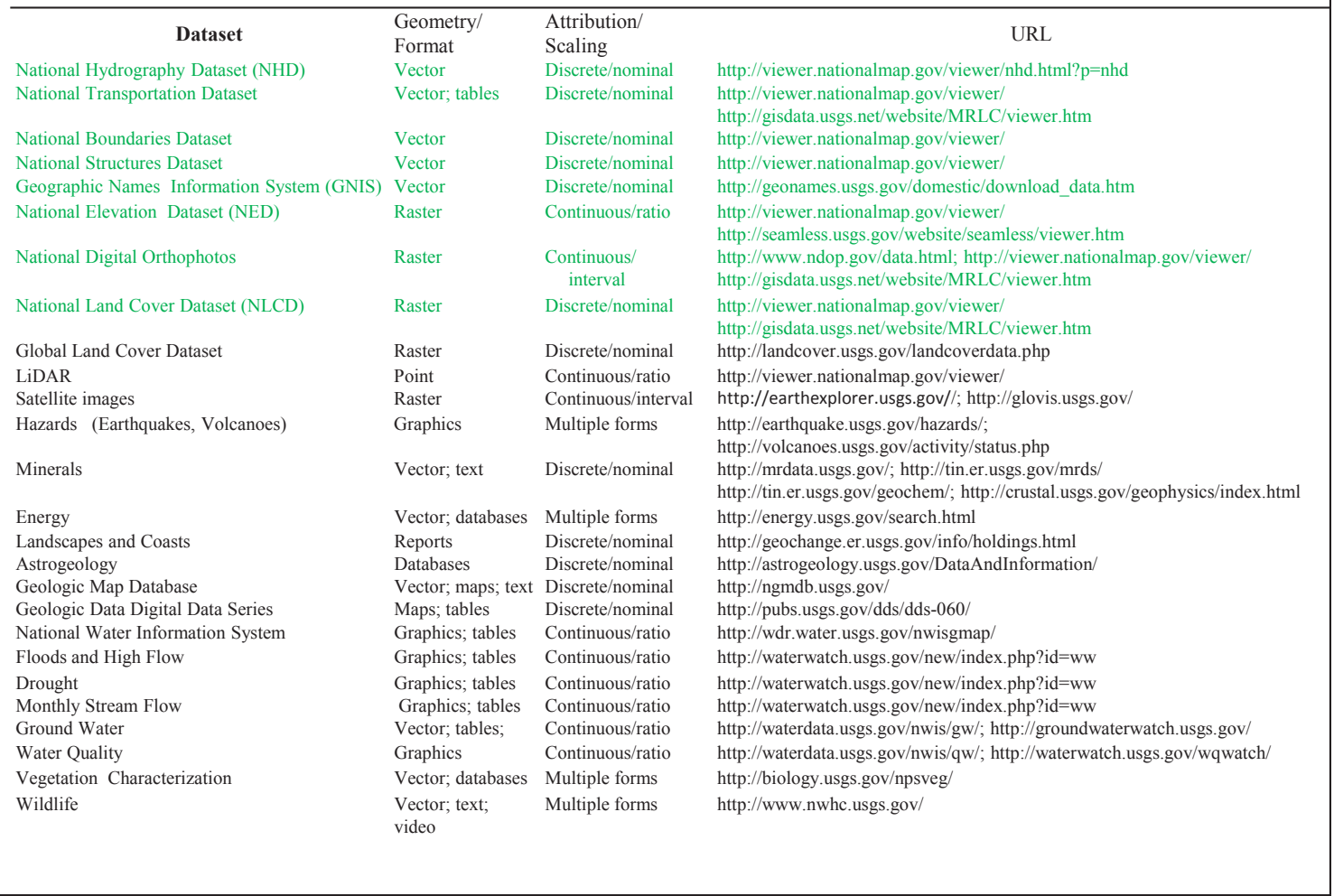

\section{The National Map - http://nationalmap.gov/}

The National Map is a collaborative effort to improve and deliver topographic information for the Nation

The goal of The National Map is to become the Nation's source for trusted, nationally consistent, integrated and current topographic information available online for a broad range of uses 


\section{The 8 Layers of The National Map}

₹USGS

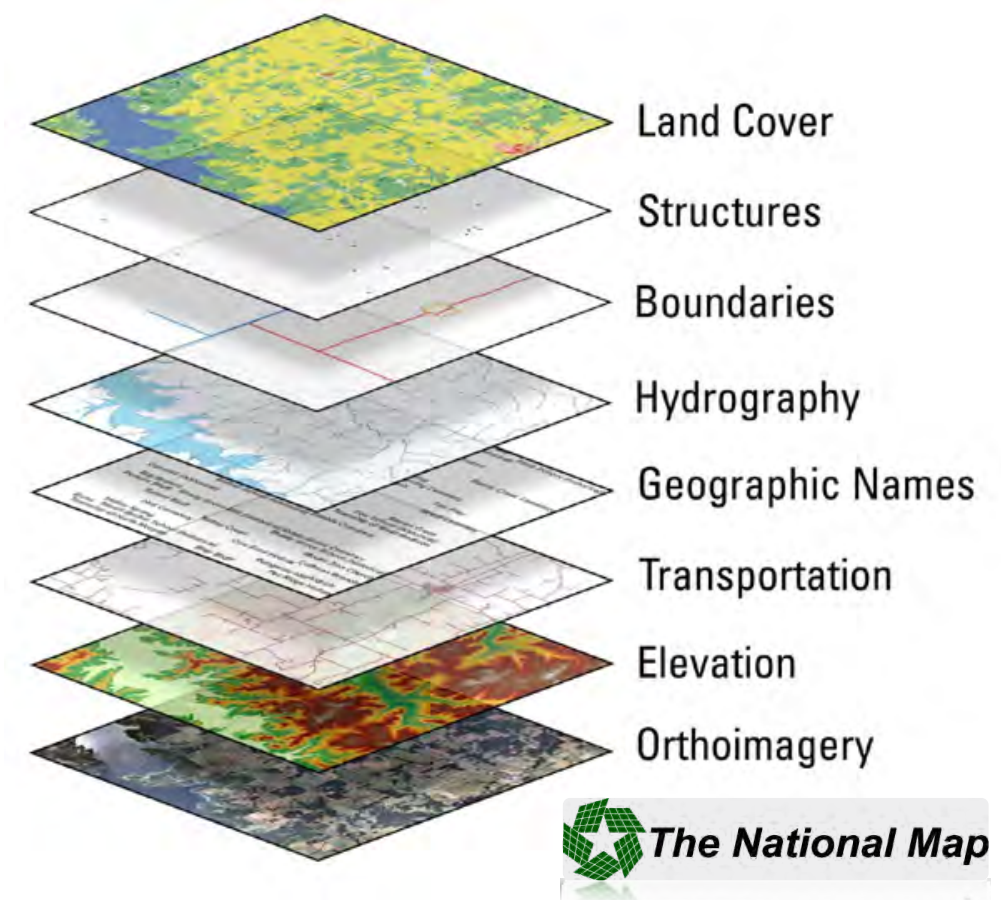

\section{Datasets of The National Map}

National Land Cover Dataset (1992, 2000, 2006)

National Elevation Dataset (1,1/3,1/9 arc-sec)

National Digital Orthophoto Dataset (multiple dates, multiple resolutions, $1 \mathrm{~m}, 1 / 3 \mathrm{~m}$ urban areas)

National Hydrography Dataset (NHD) (Medium, High, Local resolution)

Geographic Names Information System (GNIS)

National Structures Dataset

National Boundaries Dataset (US, state, county, minor civil divisions, governmental units)

National Transportation Dataset (TIGER and others) 


\section{Products of The National Map}

Data display through The National Map viewer http://viewer.nationalmap.gov/viewer/ Palanterra, joint development - National Geospacial-Intelligence Agency, ESRI, USGS

Display user selected The National Map data Data download of eight layers Mashups with other data using Keyhole Markup Language (KML)

\section{ॠUSGS}

\section{Products of The National Map}

US Topo - New 1:24,000-scale topographic maps in GeoPDF; Complete United States coverage 20092011; available for free download from USGS Map Store, beginning revision on 3-year cycle Produce more than 100 maps per day

http://nationalmap.gov/ustopo/index.html

Digital, georeferenced versions of all previous topographic maps for a specified 7.5-minute area; more than 140,000 of the 180,000 total available http://nationalmap.gov/historical/ 


\section{Semantics as a Solution}

Allows mapping of vocabularies among datasets

For example, vocabulary of USGS Digital Line Graph (DLG), DLG-Enhanced, Spatial Data Transfer Standard (SDTS), and others were used in building our semantic vocabulary

Allows data integration for query and mapping without reformatting data from various sources to a common format

Allows data use and applications not supported by GIS and GIS data models

\section{₹USGS}

\section{Building Semantics for Geospatial Data}

From scratch

Ontology

Collect data as RDF according to ontology

From Existing Data

Ontology

Convert existing data

Match data to ontology 


\section{Creating New Data with Semantics}

Develop ontology

Taxonomy of all features

Vocabulary with complete definitions

Make compatible with existing systems

Use standard vocabularies, if they exist

Compilation of all attributes and relations as

RDF triples, i.e., attributes and relations become predicates

Create data instances with geometry and ontology references

\section{ॠUSGS}

\section{New Data Example using Protégé}

Create Turtle (.ttl) file in text editor including prefixes and OWL geometry and WKT properties (use template)

Open the .ttl file in Protégé and create classes

Add individuals (instances) and geometry

Add coordinates to annotations

Link feature instances to geometry objects 


\section{Converting Legacy Data}

Conversion possibilities and methods depend on content and format of legacy data

Feature-based data are usually converted easily

Features become subjects and objects, attributes and relations become predicates

Relational data can be used to automatically form RDF triples

Rows are subjects

Columns are predicates

Cell values are objects

Tables are classes

₹USGS

\section{Conversion Example - Hydrography}

\#\# NHD feature

@ prefix geo: <http://www.opengis.net/def/geosparql/>. Define Prefixes @prefix gnis: <http://cegis.usgs.gov/rdf/gnis/> .

@prefix nhd: <http://cegis.usgs.gov/rdf/nhd/> .

<http://cegis.usgs.gov/rdf/nhd/Features/flowline/166450429> a nhd:flowline; gnis:id <http://cegis.usgs.gov/rdf/gnis/Features/737907>; nhd:enabled "1";

nhd:fCode <http://cegis.usgs.gov/rdf/nhd/fCode/46006>;

List predicates for nhd:fDate "Wed Oct 22 21:51:03 CDT 2008";

nhd:fType <http://cegis.usgs.gov/rdf/nhd/fType/460>;

nhd:flowline

nhd:flowDir <http://cegis.usgs.gov/rdf/nhd/flowDir/1>;

nhd:lengthKM 0.044;

nhd:reachCode <http://cegis.usgs.gov/rdf/nhd/reachCode/10290107000164>;

nhd:resolution 2;

nhd:shapeLength $\langle 0\rangle$;

geo:hasGeometry <http://cegis.usgs.gov/rdf/nhd/Geometries/166450429> .

<http://cegis.usgs.gov/rdf/nhd/Geometries/166450429> a geo:Geometry; geo:asWKT "LINESTRING (-93.257456099019123 37.784990808016801 0,-

$93.25769123235210137 .7852106080164280,-93.25770129901872$

Assign geometry

37.785318474682924 0)"^^<http://www.opengis.net/def/sf/wktLiteral> . as WKT 


\section{Conversion Example - Transportation}

\#\# Transportation Feature

@prefix trans: <http://cegis.usgs.gov/rdf/trans/> .

@ prefix transf: <http://cegis.usgs.gov/rdf/trans/Features/> .

@prefix transg: <http://cegis.usgs.gov/rdf/trans/Geometries/> .

@prefix geo: <http://www.opengis.net/def/geosparql/> .

Define Prefixes

<http://cegis.usgs.gov/rdf/trans/Features/22609575> a trans:roadSegment;

trans:cffcCode "A31";

trans:countyRoute "2-83";

trans:dataSecurity "5";

List predicates for

trans:distributionPolicy "E4";

trans:roadSegment

trans:fullStreetName "State Hwy N";

trans:isOneWay false;

trans:loadDate "Fri Jan 16 10:06:34 CST 2009", "Sat Mar 28 10:33:21 CDT 2009";

trans:roadClass <http://cegis.usgs.gov/rdf/trans/roadClass/10002>;

trans:shapeLength 0.0126977422205;

trans:sourceDataDesc "Attribute update from 2008 TIGER/Line Shapefiles Release";

trans:sourceDatasetID "\{1B7B3B39-5C38-4115-B429-5B0DD3DE0006\}";

trans:sourceOriginator "US Census Bureau";

trans:stCoFIPSCode "29015", "29085";

trans:stateRoute "82,N";

geo:hasGeometry <http://cegis.usgs.gov/rdf/trans/Geometries/22609575> .

<http://cegis.usgs.gov/rdf/trans/Geometries/22609575> a geo:Geometry;

geo:asWKT "LINESTRING (-93.466183999736813 38.071580000280335,-93.478876999717102

38.0719270002798)"^^<http://www.opengis.net/def/sf/wktLiteral> .

\section{Conversion Example - Raster Data}

\section{Requires identification of feature, attributes, and relationships in raster dataset}

Create new feature with supporting characteristics in RDF

Attach geometry from raster image

Currently using WKT of minimum bounding rectangle (MBR) since GML only supports full coverage and MBR of raster objects 


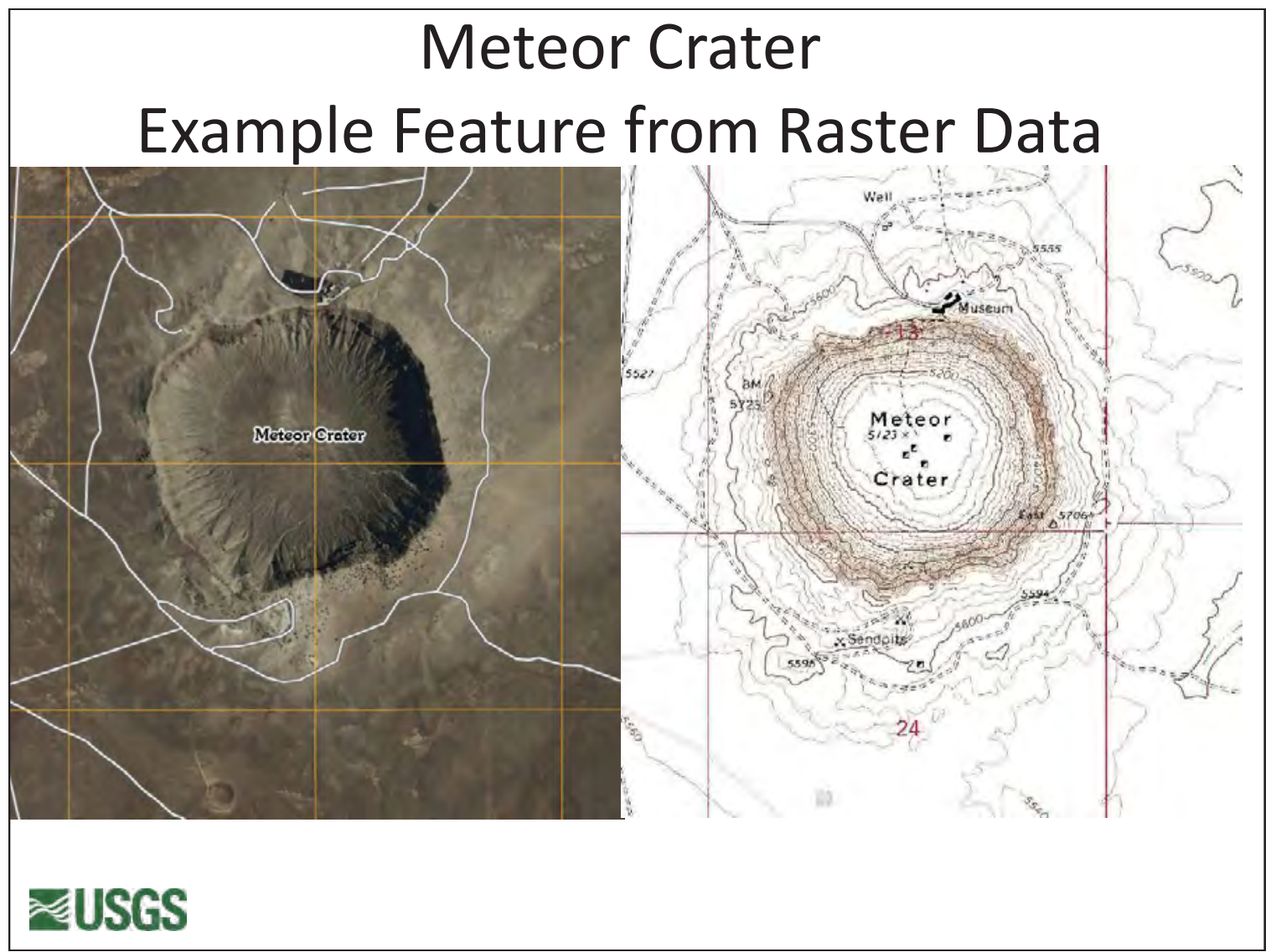

\section{Meteor Crater - Shaded Relief Image}




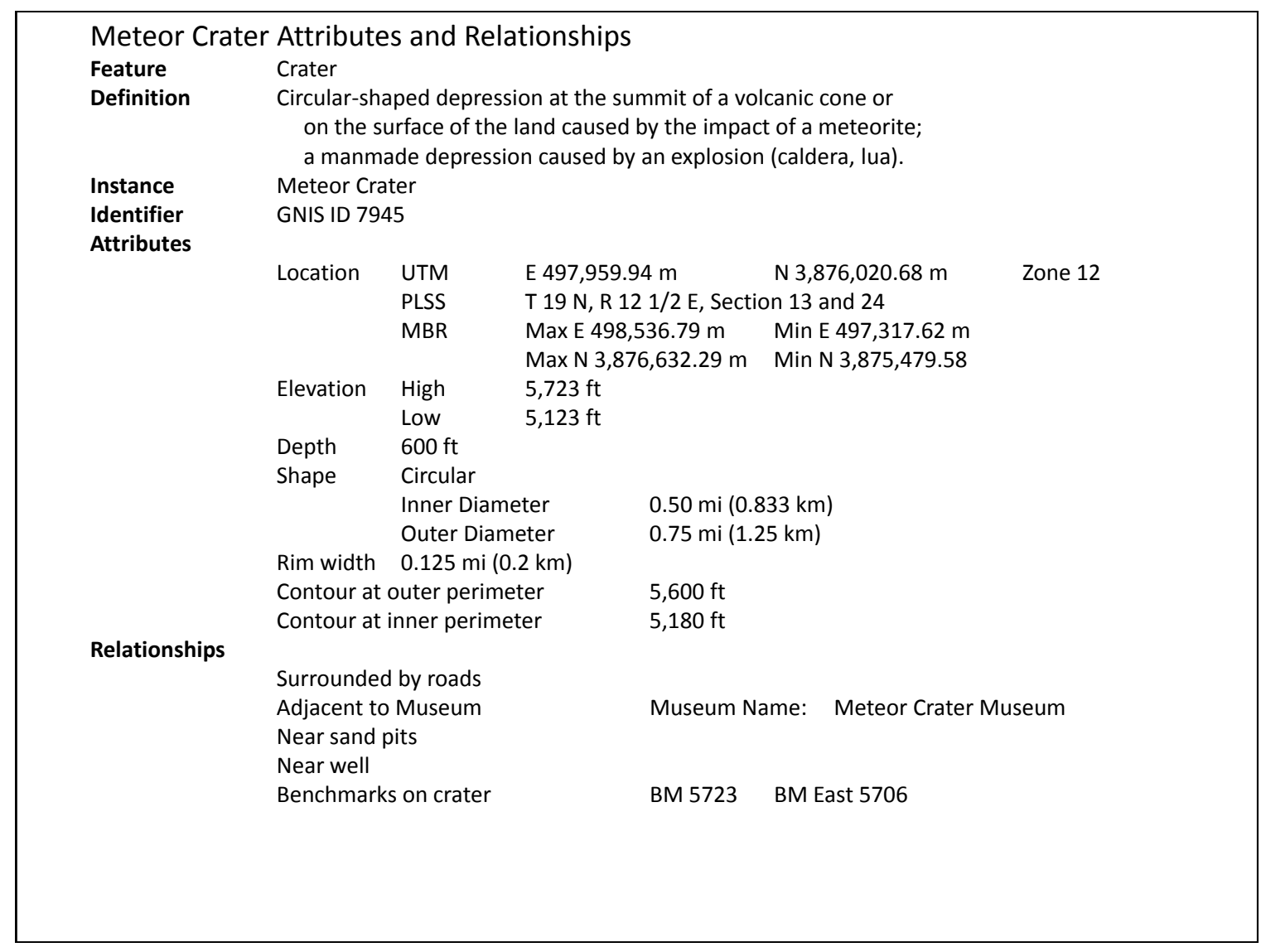

\section{Example of Meteor Crater in RDF/OWL}

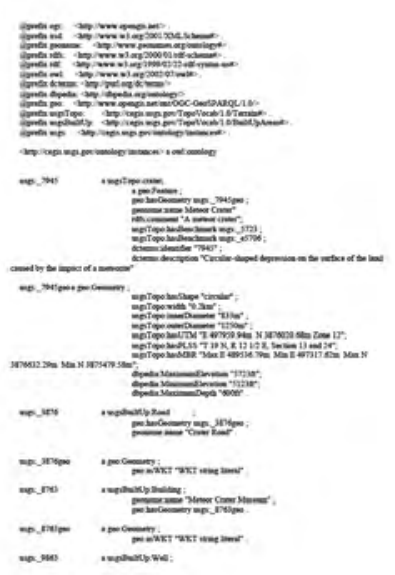




\section{Data Archive and Access}

Data are archived as RDF triplestore

Usually text-based (ASCII) triples (.ttl, RDF/XML, NTriples, ...)

Generates large data volumes, e.g., for Pomme de Terre 452,577

triples, $43 \mathrm{Mb}$

Can be optimized by making binary, e.g., Parliament Triple Store Data can be accessed by:

Query of SPARQL Endpoint (using SPARQL or GeoSPARQL)

Downloaded (remember large data volumes)

Accessed by URI for mashups with other data

Application access (concept of Open Linked Data)

DBpedia, browsing RDF data but not as queries

Access by URI from one dataset to another

\section{USGS Sample Data as RDF}

Availability

Nine test areas - converted with pre-

computed spatial relations

New conversions of the nine areas with

supporting ontology

Access to Pomme de Terre, MO watershed for this workshop 


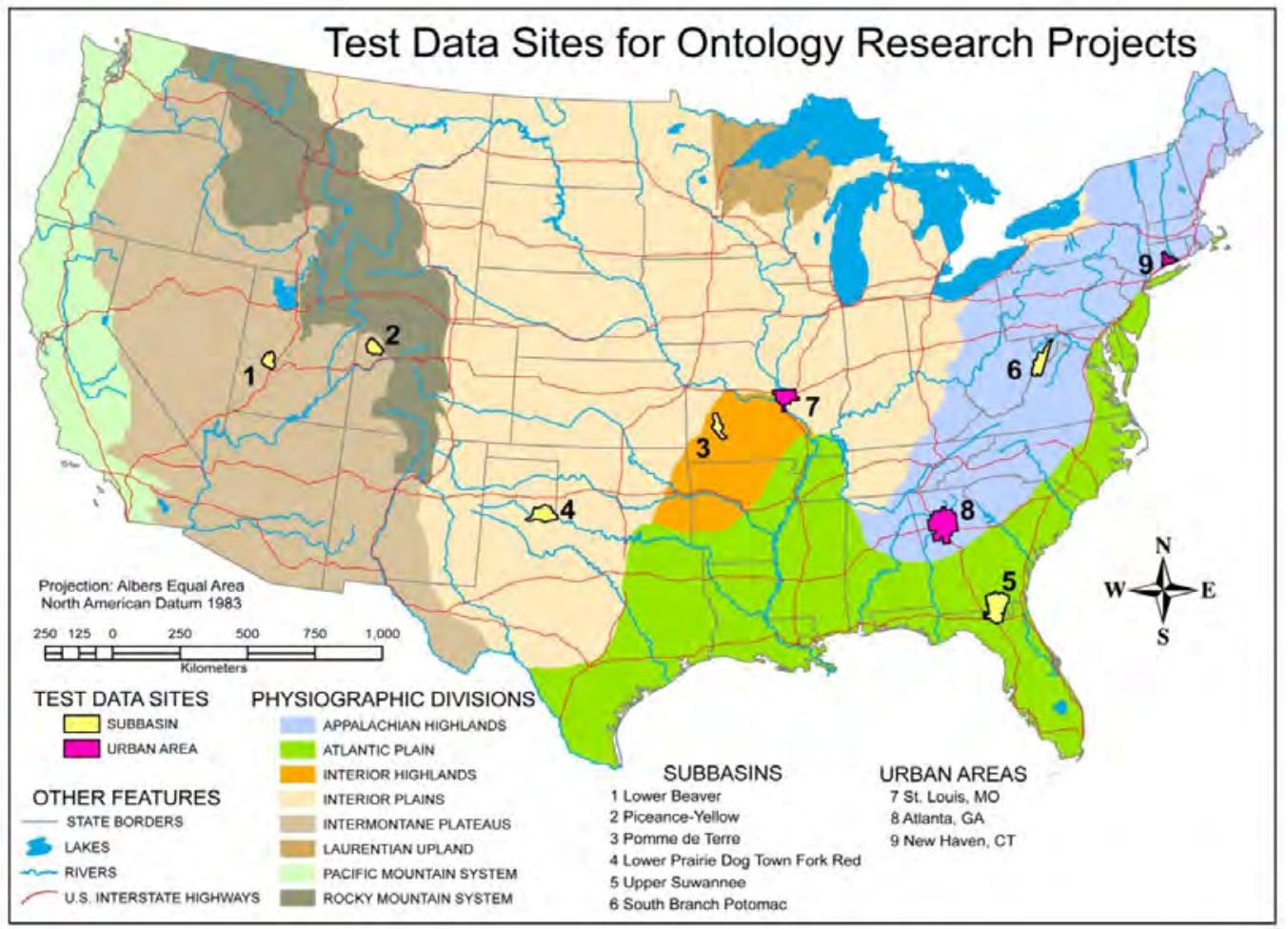

\section{Ontology for The National Map}

Taxonomy - hierarchy of feature classes

Vocabulary - feature names and definitions

Predicates - attributes and relationships

Instances - actual features with coordinates

All defined as OWL/RDF triples 


\section{Taxonomy of Feature Domains}

\section{Events}

Divisions

Built-up areas

Ecological regime

Surface water

Terrain

Domains derived from ground surveys incorporated in DLG standards

\section{₹USGS}

\section{Events}

\begin{tabular}{|llll|}
\hline Risk & & Legacy & \\
Hazard & Hazard zone & $\begin{array}{l}\text { Military history } \\
\text { Historical }\end{array}$ & $\begin{array}{l}\text { Archeological } \\
\text { site }\end{array}$ \\
Earthquake & Incident & marker & Cliff dwelling \\
Flood & Fire & Tree & Ruins \\
$\begin{array}{l}\text { Area to be } \\
\text { submerged }\end{array}$ & Restricted area & Wreck & Pictograph \\
\hline
\end{tabular}




\section{Divisions}

\begin{tabular}{|c|c|c|}
\hline \multicolumn{2}{|c|}{ Civil Units } & Boundaries \\
\hline Cadastral & Nation & Fenceline \\
\hline Parcel & Territory & Hedge \\
\hline Public Land Survey System & Reservation & Place \\
\hline Land grant & State & Region \\
\hline Homestead entry & County & Locale \\
\hline Survey line & Census & Boundary line \\
\hline Principle meridian & State & Boundary point \\
\hline Baseline & County & Hydrologic unit \\
\hline Survey point & Census county division & \\
\hline Point monument & Block group & Shipping \\
\hline Survey corner & Block & Lane \\
\hline Government unit & Tract & Traffic separation scheme area \\
\hline Municipality & Special use zone & Pilot water \\
\hline City & Time zone & Roundabout \\
\hline Town & Nature reserve & Inshore trafic zone \\
\hline Villiage & Survey line & Exclusive Economic Zone \\
\hline
\end{tabular}

\section{Built up}

\section{Built-up Category}

Transportation and warehousing

Entertainment and recreation

Utilities

Resource extraction

Structure

Agriculture and fishing

Military

Communication

Waste management

Real estate

Place of worship

Manufacturing

Institutions

Burial grounds

Disturbed surface

Trade
Number of features

in Category

60

26

16

13

12

11

10

7

7

6

6

4

3

3

3

3 


\section{Ecological Regime}

Tundra

Desert

Grassland

Scrubland

Forest

Pasture

Agricultural land

Transition area

Nature reserve

Wetland

\section{‡USGS}

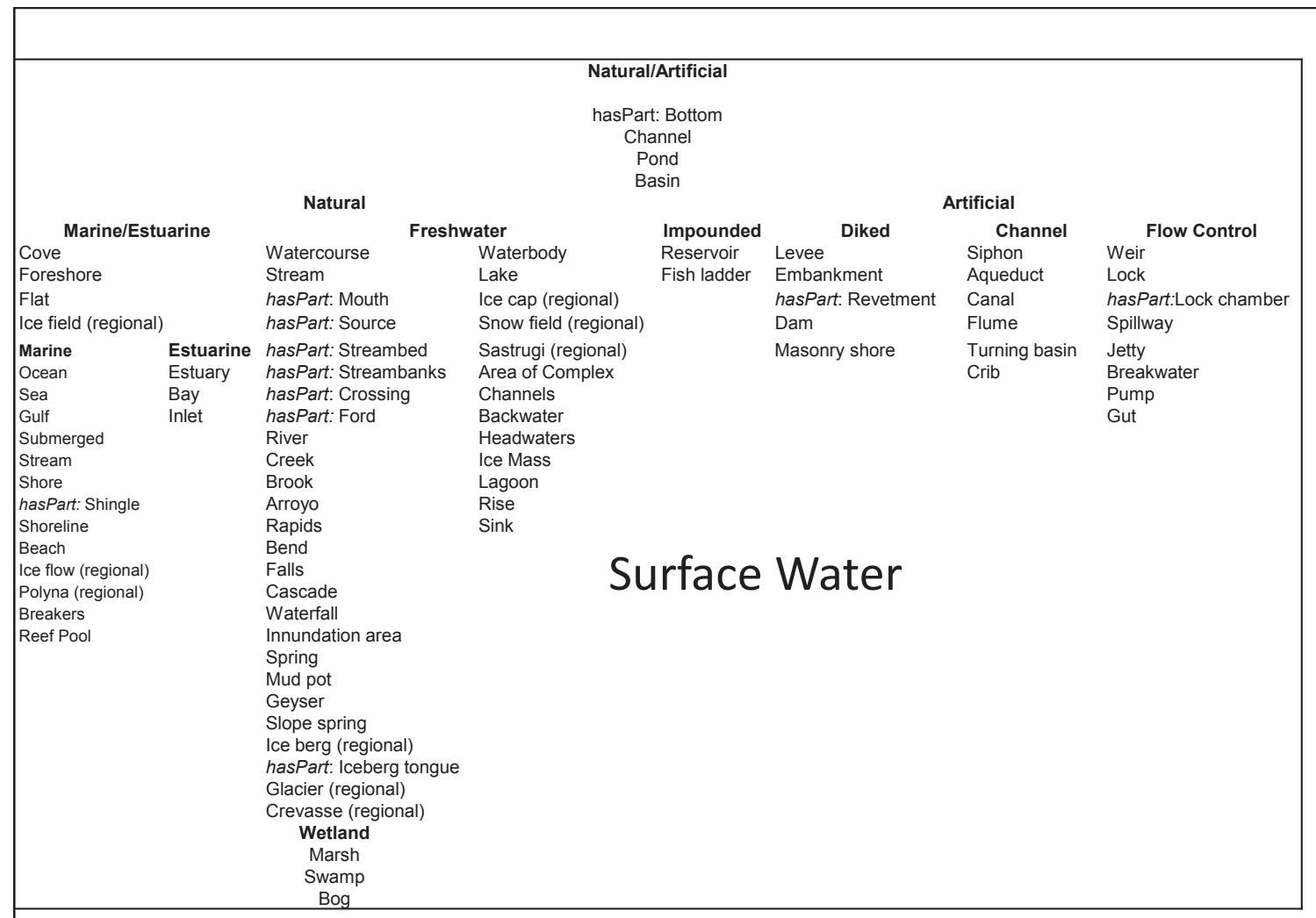




\section{Terrain includes 57 USGS landform features}

\begin{tabular}{|llll|}
\hline Arch & Divide & Isthmus & Ridge \\
Bar & Drainage basin & Karst & Ridge line \\
Basin & Dunes & Lava & Rock \\
Beach & Fault & Mineral pile & Salt pan \\
Bench & Flat & Moraine & Shaft \\
Cape & Floodplain & Mount & Sink \\
Catchment & Fracture & Mountain range & Summit \\
Cave & Fumarole & Peak & Talus \\
Chimney & Gap & Peneplain & Terrace \\
Cirque & Glacial & Peninsula & Valley \\
Cliff & Ground surface & Pinnacle & Volcano \\
Coast & Hill & Plain & Wash \\
Continent & Incline & Plateau & \\
Crater & Island & Quicksand & \\
Delta & Island cluster & Reef & \\
\hline
\end{tabular}

ÆUSGS

\section{Topographic Vocabulary}

Examples from:

Events

Divisions

Built up

Ecological regime

Surface water

Terrain

Available from Ontology Project Webpage:

http://cegis.usgs.gov/ontology.html 


\section{Ontologies - Reuseable Vocabularies}

@prefix geonames: <http://www.geonames.org/ontology\#> .

@ prefix rdfs: <http://www.w3.org/2000/01/rdf-schema\#>.

@ prefix rdf: <http://www.w3.org/1999/02/22-rdf-syntax-ns\#>.

@prefix owl: <http://www.w3.org/2002/07/owl\#> .

@ prefix dcterms: <http://purl.org/dc/terms/>

@prefix dbpedia: <http://dbpedia.org/ontology/>

@prefix geo: <http://www.opengis.net/ont/OGC-GeoSPARQL/1.0/>

@prefix usgsTopo: <http://cegis.usgs.gov/TopoVocab/1.0/Terrain\#>.

@prefix usgs: <http://cegis.usgs.gov/ontology/instances\#> .

\section{Built-up Vocabulary}

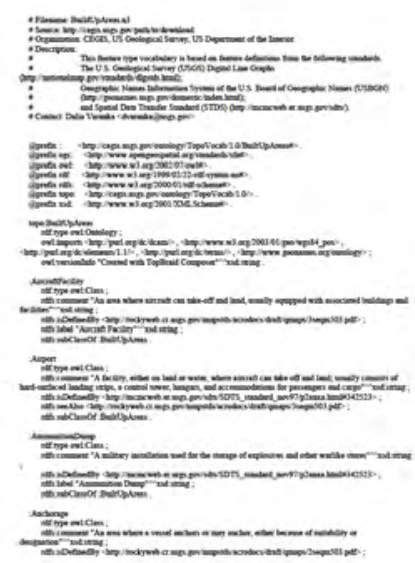




\section{Terrain Vocabulary}

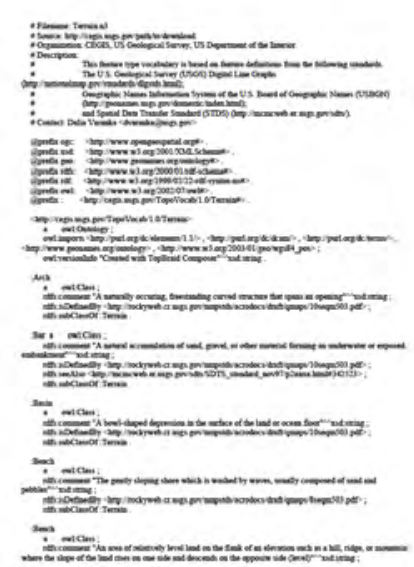

\section{Querying USGS Sample Data with SPARQL and GeoSPARQL}




\section{SPARQL Endpoint}

A URL that allows access to an RDF triplestore

USGS SPARQL Endpoint for Topographic Data

http://usgs-ybother.srv.mst.edu:8890/parliament

‡USGS

\section{Triplestore of USGS Data}

Collection of RDF triples for our 9 research test areas.

Data will include names, hydrography, transportation, boundaries, structures, land cover, geomorphic features (from elevation) Accessible from SPARQL Endpoint SPARQL queries use the ontology 


\title{
SPARQL Query Example
}

\author{
\#\# SPARQL Query \\ PREFIX gnis: <http://cegis.usgs.gov/rdf/gnis/> . \\ Prefixes defining shorthand \\ PREFIX rdfs: <http://www.w3.org/2000/01/rdf-schema\#> . notation for URIs \\ SELECT ?name ?mapName \\ WHERE \\ \{ \\ ?x a gnis:gnisFeature . \\ ?x rdfs:label ?name. \\ ?x gnis:mapName ?mapName . \\ \} \\ Select clause defining variables \\ (name and mapName in this \\ case) \\ Match features and bind to variables
}

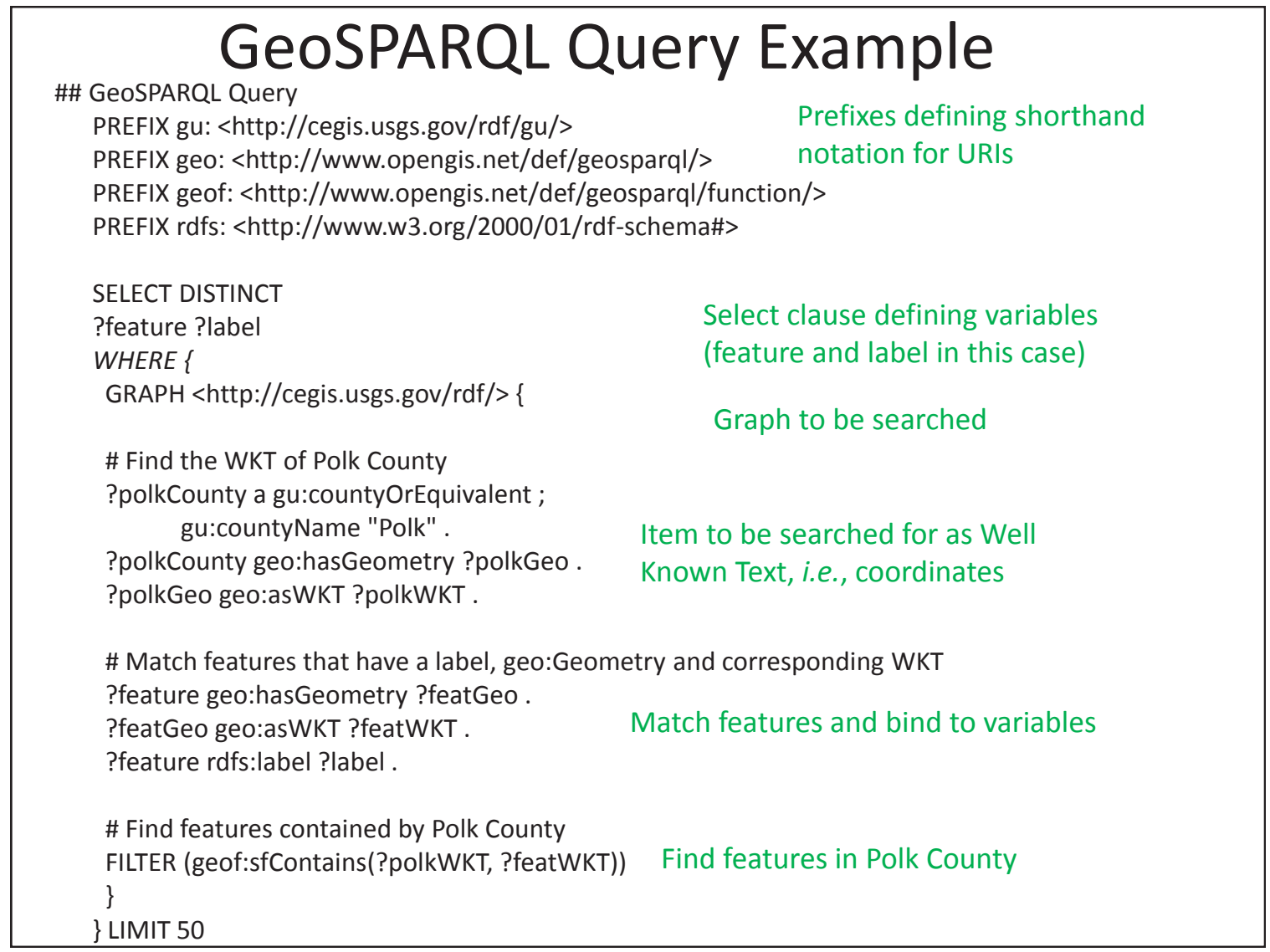




\section{Using USGS Data with Other Data}

Use of URIs in USGS data and URIs from other data provide access

User (computer program that does linking) must determine if data are compatible and make sense

USGS data join the Open Linked Data community

\section{ॠUSGS}

\section{Linking Data}

Data are linked across triplestores by URIS

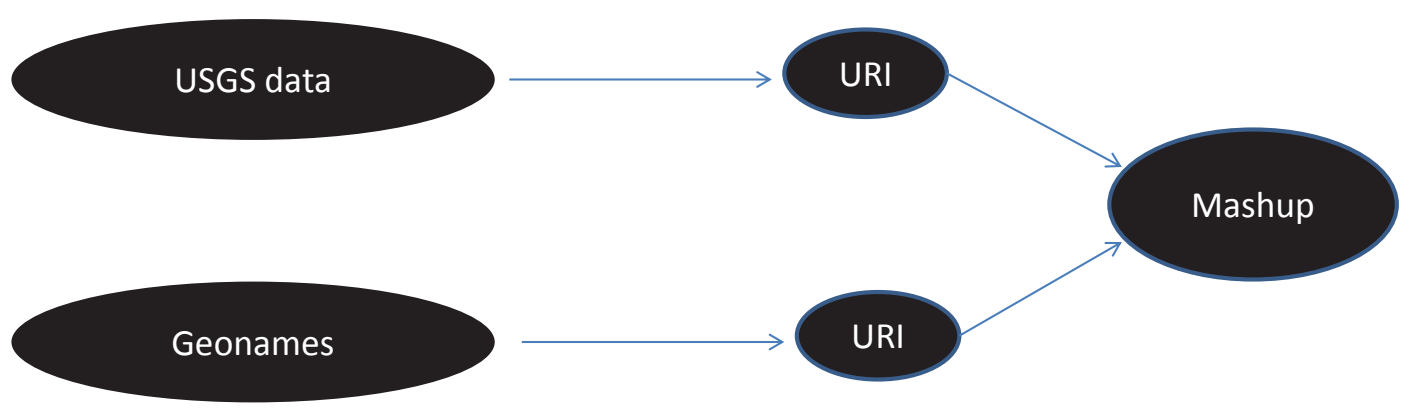




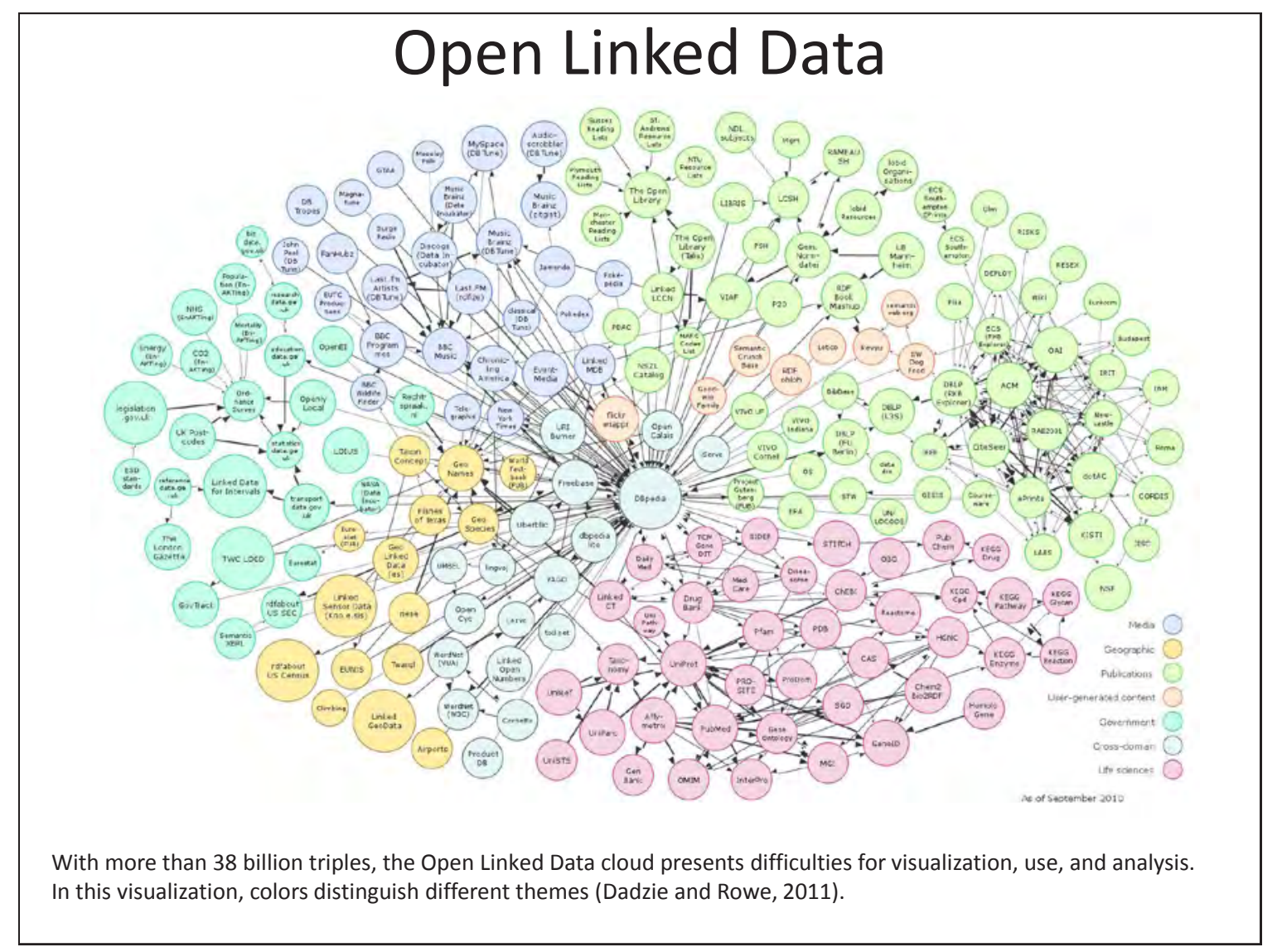

\section{USGS Research Needs in Geosemantics}

Gazetteer access to geospatial features and data

Geospatial operators and ontology-driven processes that work with RDF

Direction, distance, overlay, buffer, generalization, mapping and display, geospatial analysis, visualization terrain analysis, map algebra

Automated feature identification in raster data including unnamed features, e.g., using ontology design patterns and feature identification software

\section{ఇUSGS}




\section{Future of Semantic Data at USGS}

Convert all data for the nine test areas

Build raster features, e.g., geomorphic named and unnamed

Design and build gazetteer interface

Design and build operators for semantic data

Convert all data for The National Map to semantics

₹USGS

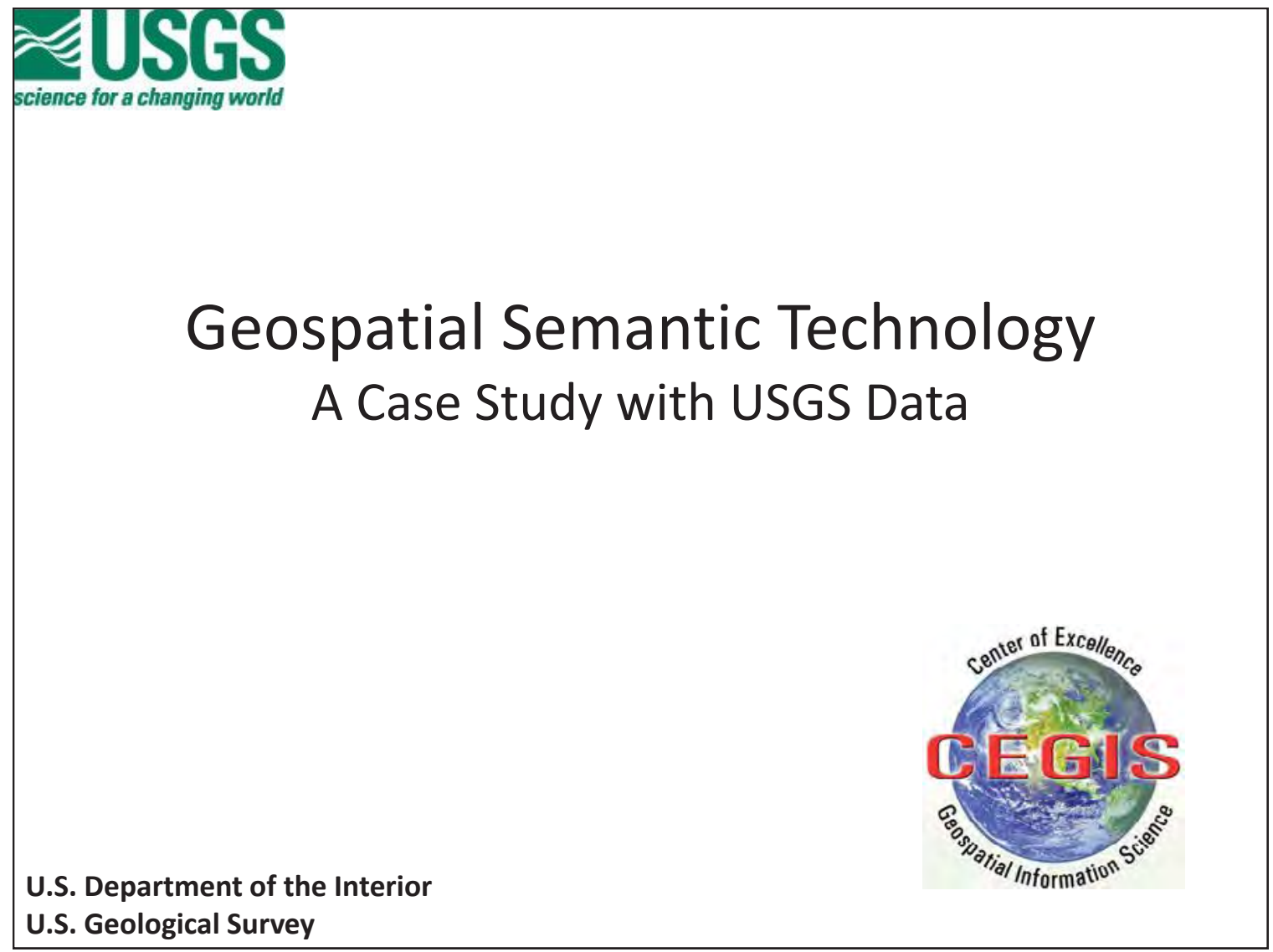




\section{Accessing Topographic Data Triples}

The building of ontology for The National Map topographic data will be reviewed. Demonstrations of data from The National Map in triple format, accessible through the triple store endpoint and custom interface, will be shown by designing some commonly used SPARQL and GeoSPARQL types of queries.

\section{Geospatial Semantic Technology Hands-on with RDF and SPARQL David Mattli<dmattli@usgs.gov>}

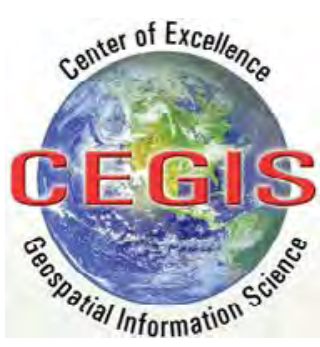




\section{Outline}

- Resource Description Framework

- Syntax

- RDF query examples

David Mattli <dmattli@usgs.gov>

\section{₹USGS}

\section{Resource Description Framework}

RDF models data using triples: Subject-Predicate-Object 


\section{The sky is blue.}

- If we want to store the idea of a blue sky we choose a subject to represent "the sky"

- We select a predicate to represent "has the color"

- And we choose an object that represents the concept of "blue"

\section{¿USGS}

\section{Triple Example}

- "The sky is blue" in triple form might look like this:

<http://example.com/sky><http://example.com/hasColor > <http://example.com/blue> .

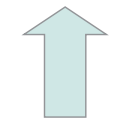

Subject

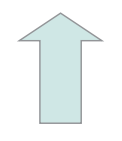

Predicate

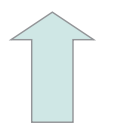

Object

- Each part is called an "RDF term"

- Each RDF term is separated by (at least) a space

- The triple ends with a period 


\section{URIs vs URLs}

- Each of the terms from this example

<http://example.com/sky><http://example.com/hasColor> <http://example.com/blue>.

are URIs.

- They look very similar to URLs but the URIs used in RDF triples do not necessarily specify locations on the web

- URIs are used as unique names

\section{¿USGS}

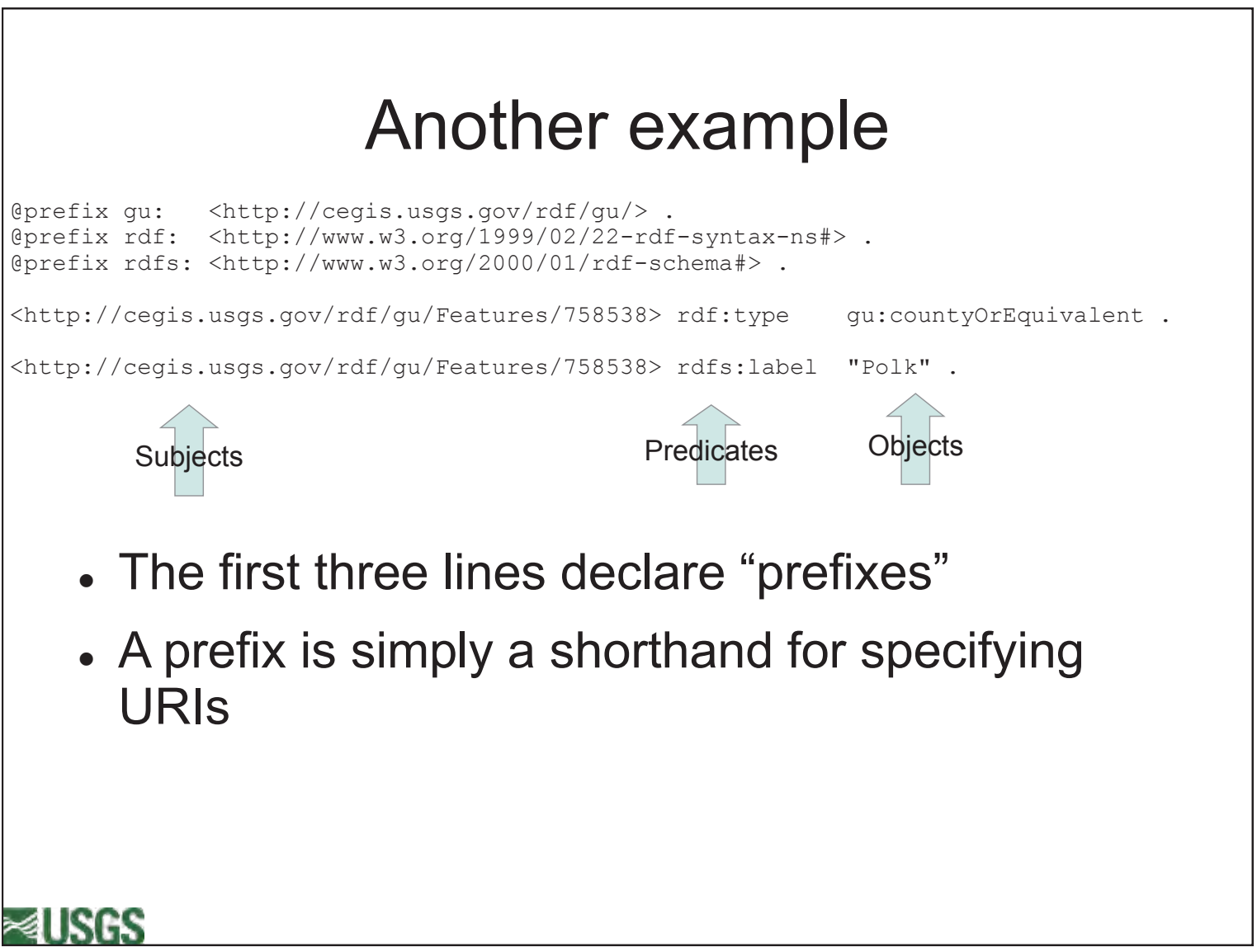




\section{Another example}

@prefix gu: <http://cegis.usgs.gov/rdf/gu/> .

aprefix rdf: <http://www.w3.org/1999/02/22-rdf-syntax-ns\#>.

eprefix rdfs: <http://www.w3.org/2000/01/rdf-schema\#> .

<http://cegis.usgs.gov/rdf/gu/Features/758538> rdf:type gu:countyOrEquivalent.

<http://cegis.usgs.gov/rdf/gu/Features/758538> rdfs:label "Polk" .

Subjects Predicates Objects

- Same "Subject Predicate Object" structure

- But now we have two triples

- The first describes a "type"

- The second describes the name or label of the

¿USGS feature

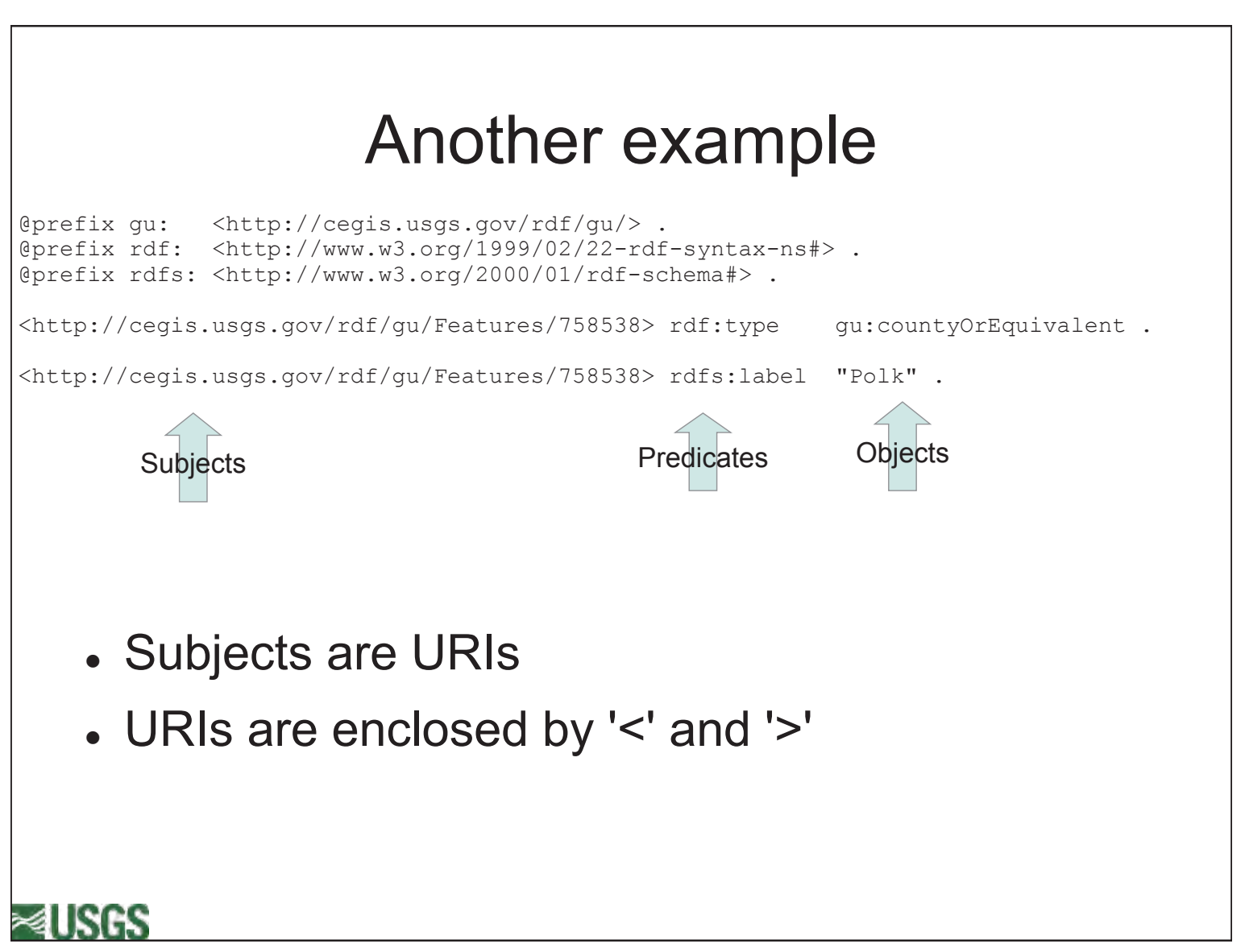




\section{Another example}

@prefix gu: <http://cegis.usgs.gov/rdf/gu/> .

aprefix rdf: <http://www.w3.org/1999/02/22-rdf-syntax-ns\#> .

@prefix rdfs: <http://www.w3.org/2000/01/rdf-schema\#>.

<http://cegis.usgs.gov/rdf/gu/Features/758538> rdf:type gu:countyOrEquivalent.

<http://cegis.usgs.gov/rdf/gu/Features/758538> rdfs:label "Polk" .

Subjects

Predicates Objects

- Predicates are also URIs

- The first three lines declare "prefixes"

- A prefix is simply a shorthand for specifying URIS

₹USGS

\section{Another example}

@prefix gu: <http://cegis.usgs.gov/rdf/gu/> .

@prefix rdf: <http://www.w3.org/1999/02/22-rdf-syntax-ns\#> .

aprefix rdfs: <http://www.w3.org/2000/01/rdf-schema\#>.

<http://cegis.usgs.gov/rdf/gu/Features/758538> rdf:type gu:countyOrEquivalent

<http://cegis.usgs.gov/rdf/gu/Features/758538> rdfs:label "Polk" .

- The object of this triple is a URI using a prefix

- This URI is the name attached to the concept of a "county or equivalent"

- The second triple is a little different 


\section{Another example}

@prefix gu: <http://cegis.usgs.gov/rdf/gu/> .

@prefix rdf: <http://www.w3.org/1999/02/22-rdf-syntax-ns\#> .

@prefix rdfs: <http://www.w3.org/2000/01/rdf-schema\#> .

<http://cegis.usgs.gov/rdf/gu/Features/758538> rdf:type gu:countyOrEquivalent.

<http://cegis.usgs.gov/rdf/gu/Features/758538> rdfs:label "Polk" .

- The object of a triple can be either a URI or a literal value

- The object of this triple is a string literal value

- Literal values can also be numbers, dates, geometries, etc

\section{₹USGS}

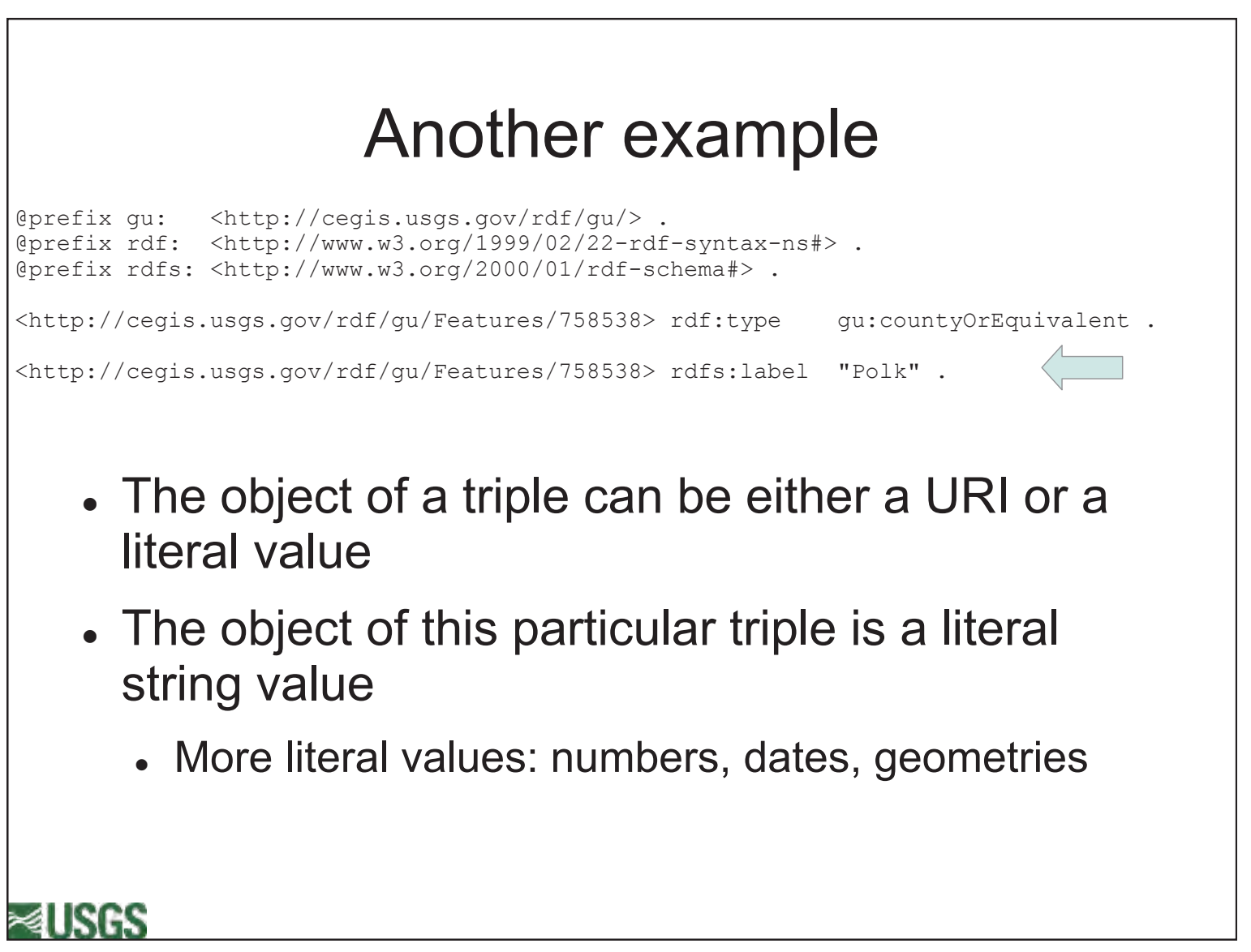




\section{Questions?}

@prefix gu: <http://cegis.usgs.gov/rdf/gu/>.

aprefix rdf: <http://www.w3.org/1999/02/22-rdf-syntax-ns\#> .

eprefix rdfs: <http://www.w3.org/2000/01/rdf-schema\#> .

<http://cegis.usgs.gov/rdf/gu/Features/758538> rdf:type gu:countyOrEquivalent .

<http://cegis.usgs.gov/rdf/gu/Features/758538> rdfs:label "Polk" .

- Any questions about RDF, prefixes, or triples?

æUSGS

\section{Triplestores}

- A collection of triples is called a "graph"

- A program that stores graphs is called a "triplestore"

- Triplestores also execute queries on graphs

- The RDF query language is called SPARQL (sparkle) 


\section{SPARQL}

Here is an example query

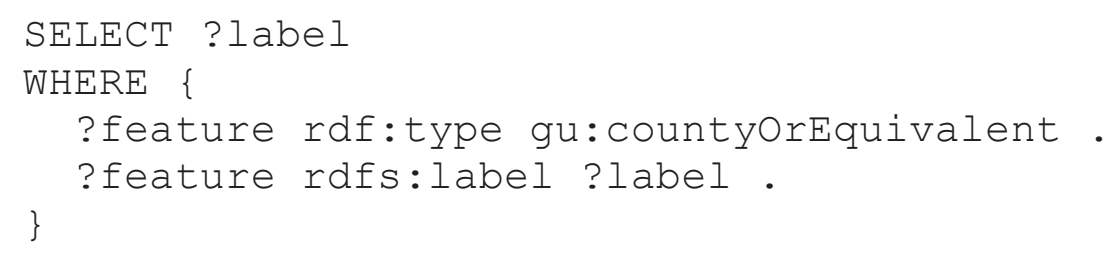

- The SPARQL query language allows you query a triplestore for RDF terms(subjects, predicates or objects)

- Next we will examine the parts of this query

\section{EUSGS}

\section{SPARQL}

\section{SELECT ? label}

WHERE \{

?feature rdf:type gu:countyOrEquivalent.

?feature rdfs:label ?label. \}

- The first part of this query is the "SELECT clause"

- The "SELECT" is followed by a space separated list of "variables"

- A "variable" is a name prefixed by a '?' 


\section{SPARQL - Variables}

\section{SELECT ?label}

WHERE \{

?feature rdf:type gu:countyOrEquivalent .

?feature rdfs:label ?label. \}

- SPARQL variables are names that we give to the RDF terms we are querying for

- This query has one variable: ?label

- Variables are arbitrary identifiers

\section{ZUSGS}

\section{SPARQL - Graph Patterns}

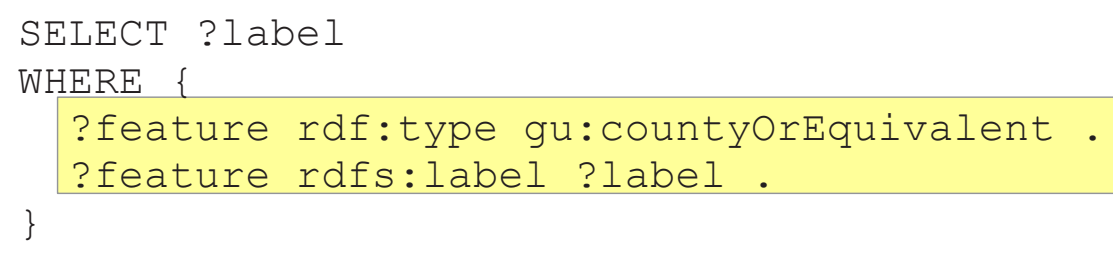

- The second part of a SPARQL query is the "graph pattern"

- The "graph pattern" is a list of "triple patterns"

- In this example there are two triple patterns 


\section{SPARQL - Graph Patterns}

SELECT ?label

WHERE \{

?feature rdf:type gu:countyOrEquivalent .

?feature rdfs:label ?label. \}

- Each "triple pattern" has the now familiar Subject-Predicate-Object structure

- Except now one or more of the RDF terms may be replaced by a variable

\section{EUSGS}

\section{SPARQL - Graph Patterns}

SELECT ? label

WHERE \{

?feature rdf:type gu:countyOrEquivalent.

?feature rdfs:label ?label. \}

- A query is executed by searching a graph in a triplestore for possible substitutions for the variables in a "triple pattern"

- The highlighted pattern would match

<http://cegis.usgs.gov/rdf/gu/Features/758538>rdf:type gu: countyorEquivalent.

but not

<http://cegis.usgs.gov/rdf/gu/Features/766568> rdf:type gu:minorCivilDivision. 


\section{SPARQL - Graph Patterns}

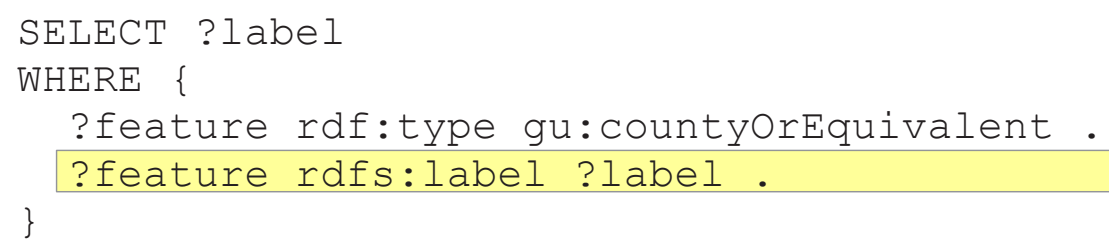

- And this pattern would match any ?feature with an rdfs:label

\section{₹USGS}

\section{SPARQL - Graph Patterns}

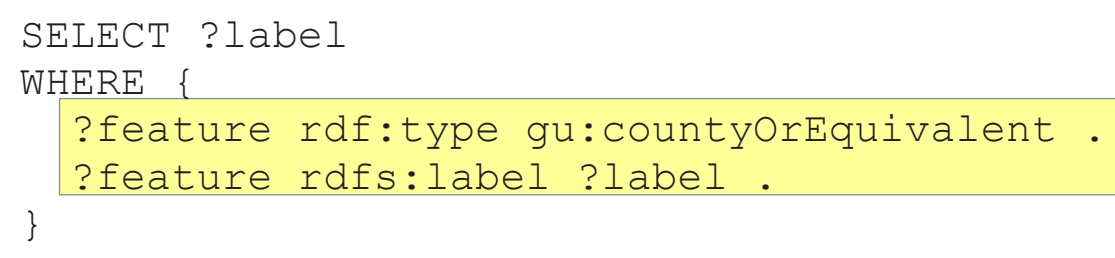

- Because the same ?feature variable is used in both triple patterns this query searches for the label of a subject that has the type " "gu: countyOrEquivalent" 


\section{SPARQL}

- Next we will try and execute this SPARQL query.

- Any questions?

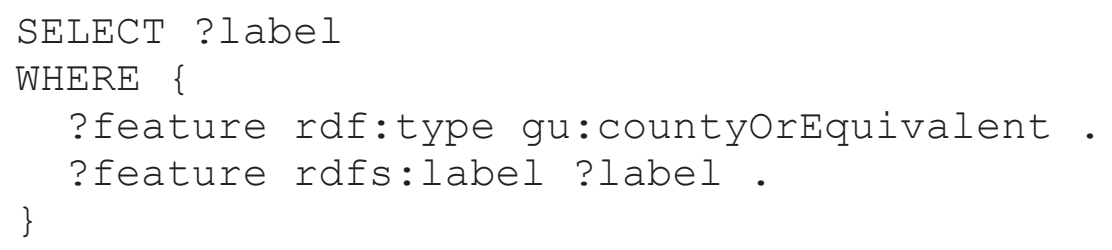

¿USGS

\section{SPARQL Query}

- Try executing a SPARQL query

- Enter this URL in your web browser:

http://usgs-ybother.srv. mst.edu/viz/

You should see something like this:

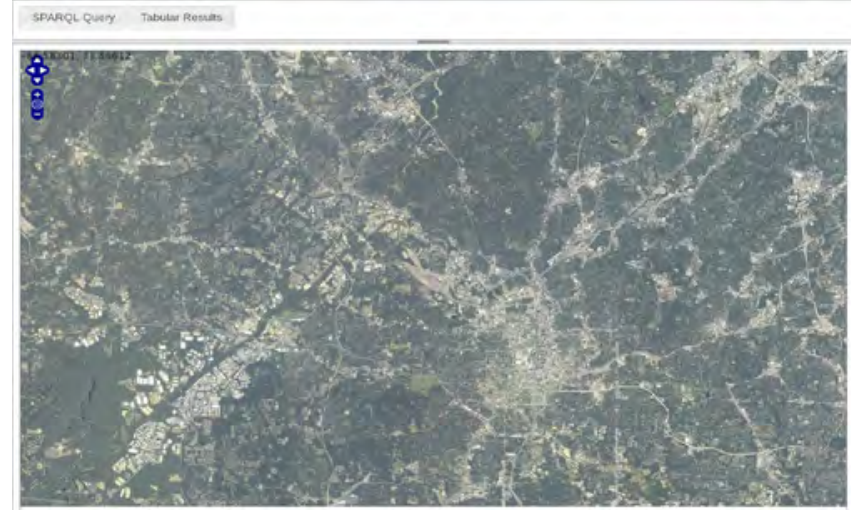




\section{SPARQL Query}

http://usgs-ybother.srv.mst.edu/viz/

- Click on the "SPARQL Query" button

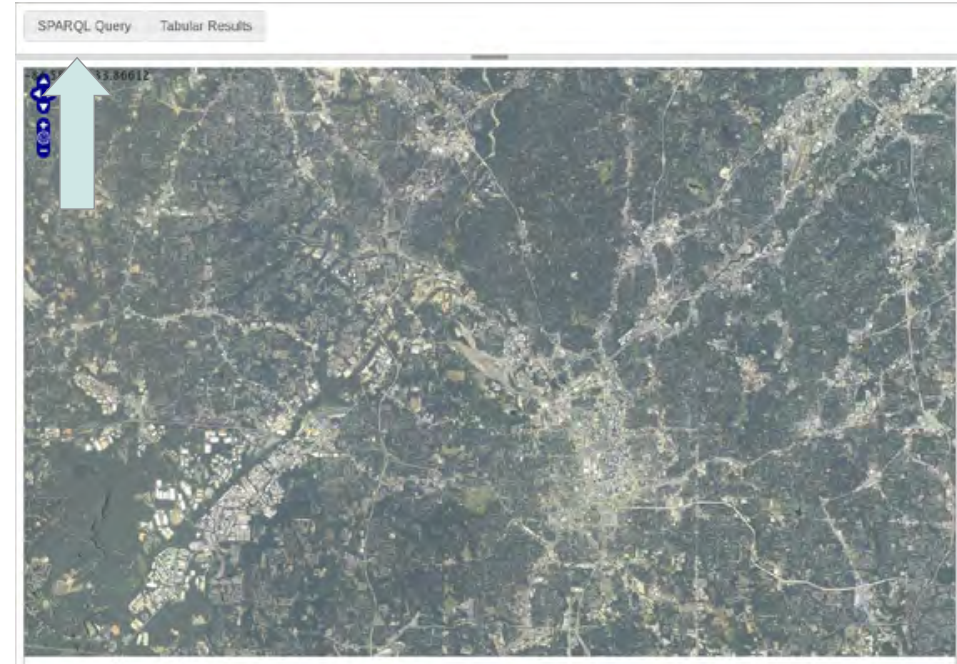

\section{SPARQL Query}

http://usgs-ybother.srv.mst.edu/viz/

- You should see a page like this:

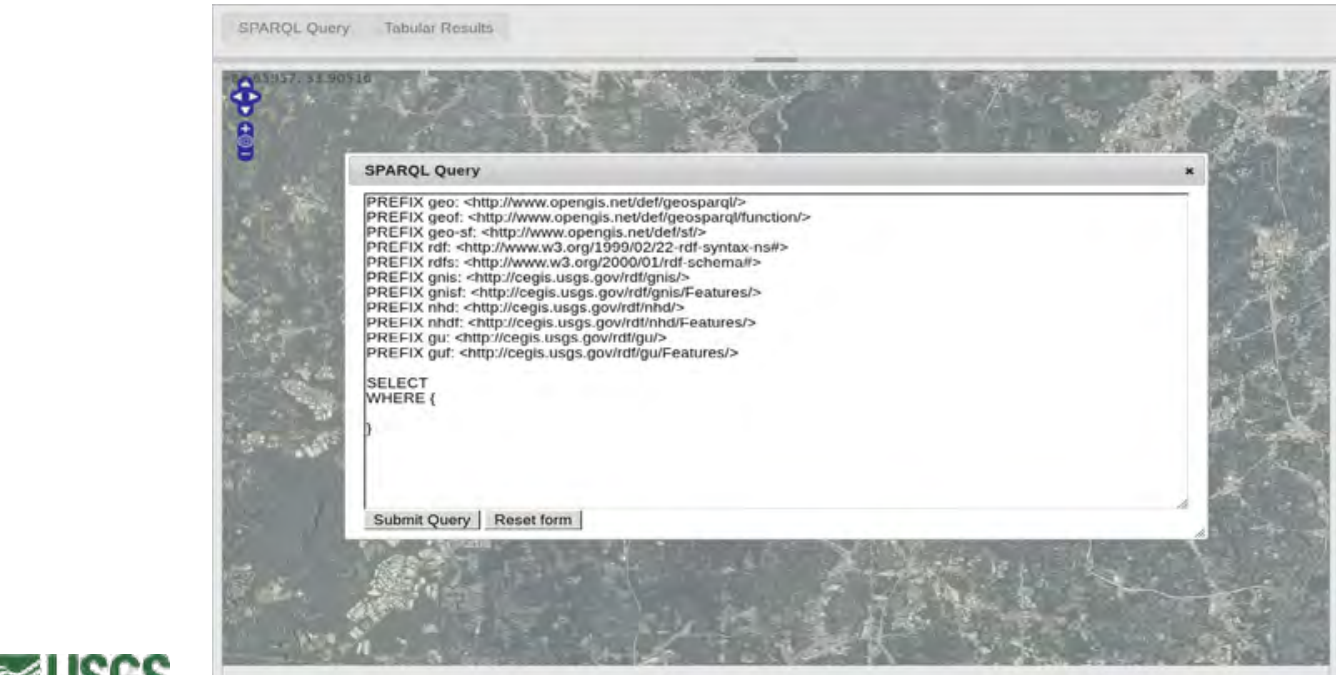




\section{SPARQL Query}

\section{http://usgs-ybother.srv.mst.edu/viz/}

\section{- Now enter the query we saw earlier}

SPARQL QUERY

PREFIX geo: <http:I/www.opengis,net/det/geosparq//>

PREFIX geof: <http://www.opengis.net/def/geosparql/function/>

PREFIX geo-sl: shttp://www.opengis.net/def/s//>

PREFIX rdf: <http///www.w3.org/1999/02/22-rdf-syntax-nstt>-

PREFIX rdts: shttp://Www w3.org/2000/01/rdt-schematts

PREFIX gnis: <http//cegis.usgs.gov/rdf/gnis/>

PREFIX gnisf: shttp://cegis.usgs.gov/rdf/gnis/Features/>

PREFIX nhd: chttp://cegis.usgs.gov/rdt/nhd/s

PREFIX nhdi: <http://cegis.usgs.gov/rdt/nhd/Features/>

PREFIX gu: <http://cegis.usgs.gov/rdf/gu/>

PREFIX gut: <http://cegis.usgs.gov/rdif/gu//Features/>

SELECT ?label

WHERE \{

?teature rdftype gu:countyOrEquivalent

?lealure rdis.label ?label .

\section{SPARQL Query}

\section{http://usgs-ybother.srv.mst.edu/viz/}

\section{- And click "Submit Query" when it is all entered}

SPARQL QuEry

PREFIX geo: <http://www.opengis.net/det/geosparq//>

PREFIX geof: <http//www.opengis.net/def/geosparql/function/>

PREFIX geo-sl: <http://www.opengis.net/def/s//>

PREFIX rdf: <http:/www.w3.org/1999/02/22-rdf-syntax-nstt:

PREFIX rdfs: <http://www w3.org/2000/01/rdf-schematts

PREFIX gnis: <http//cegis.usgs.gov/rdf/gnis/>

PREFIX gnist: shttp://cegis.usgs.gov/rdt/gnis/Features/>

PREFIX nhd: chttp://cegis.usgs.gov/rdt/nhd/s

PREFIX nhdi: <http://cegis.usgs.gov/rdt/nhd/Features/>

PREFIX gu: <http://cegis.usgs.gov/rdf/gu/>

PREFIX gut: <http://cegis.usgs.gov/rdf/gu/Features/>

SELECT ?label

WHERE \{

?teature rdftype quicountyorEquivalent

?lealure rdis:label ?label . 


\section{SPARQL Query}

http://usgs-ybother.srv.mst.edu/viz/

- Once the dialog closes, click on "Tabular Results"

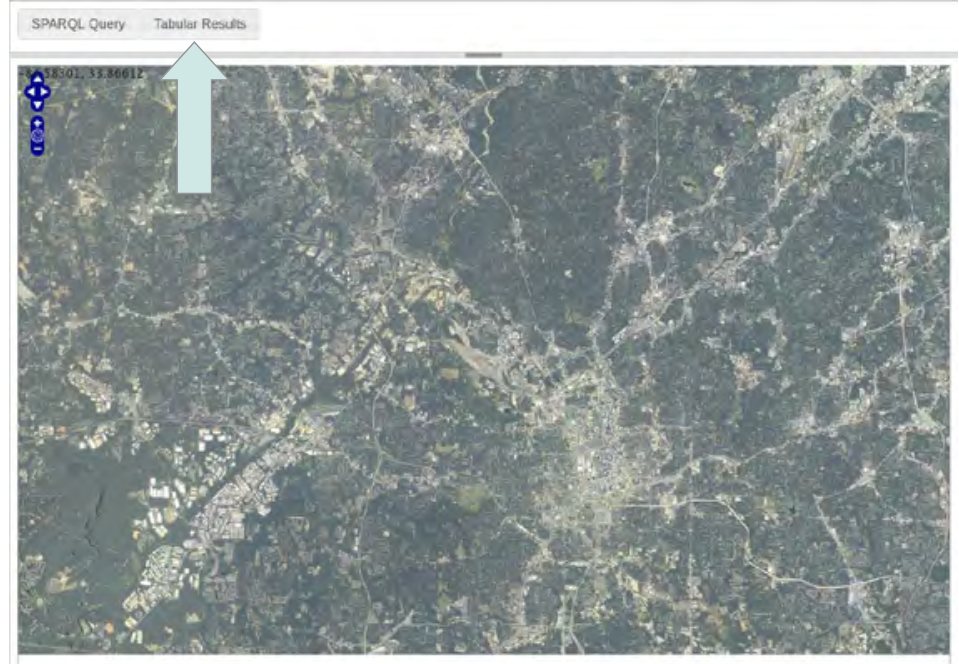

\section{SPARQL Query}

http://usgs-ybother.srv.mst.edu/viz/

- The "Tabular Results" dialog should look like:

$\mathbf{x}$

\section{label}

Webster

Polk

Hickory

Greene

Dallas

Benton 


\section{Any Questions?}

http://usgs-ybother.srv.mst.edu/viz/

- The "Tabular Results" dialog should look like:

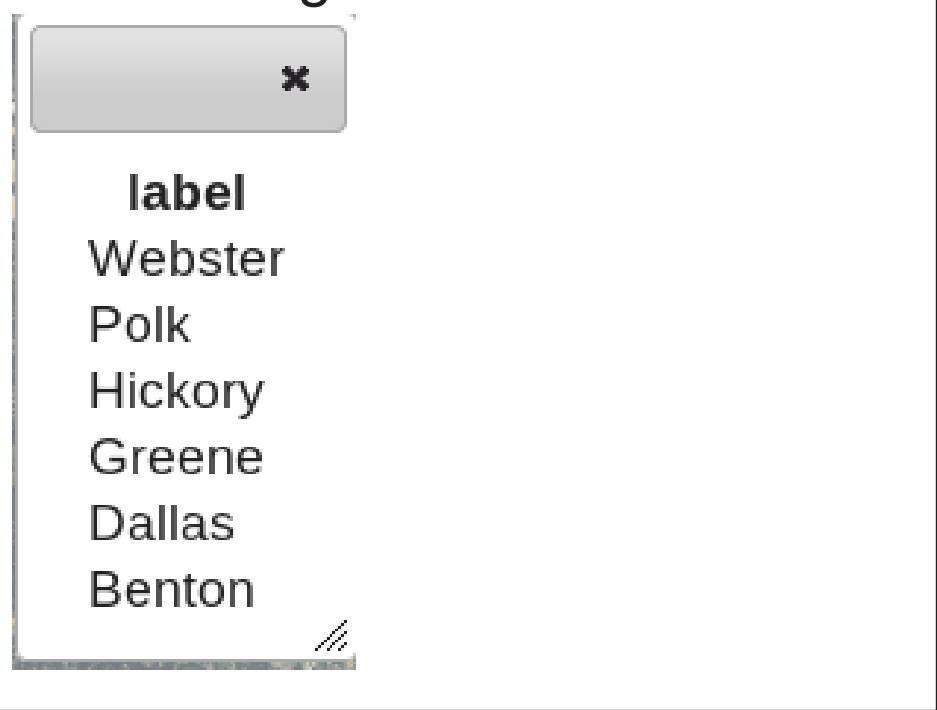

\section{GeoSPARQL}

- GeoSPARQL is an extension of SPARQL

- Associates a Geometry with a feature using geo:hasGeometry

<http://cegis.usgs.gov/rdf/nhd/Features/102204610> rdf:type nhd:flowline .

<http://cegis.usgs.gov/rdf/nhd/Features/102204610> geo:hasGeometry

<http://cegis.usgs.gov/rdf/nhd/Geometries/102204610> .

<http://cegis.usgs.gov/rdf/nhd/Geometries/102204610> rdf:type geo:Geometry .

<http://cegis.usgs.gov/rdf/nhd/Geometries/102204610> geo:asWKT

"LINESTRING (-93.387722032150236 38.166983407423857 0,-93.387682298816969

$38.1675392074229760,-93.38861943214885738 .168476474088209$ 0,-93.391319032144679

$38.1697348740862590,-93.39676843213624138 .1719242740828690,-93.398635898799967$

$38.1724902740819520,-93.39899029879944738 .172600607415120,-93.399145698799202$

38.172711207414977 0,-93.399287298798981 $38.1725742074151530,-93.399409832132108$

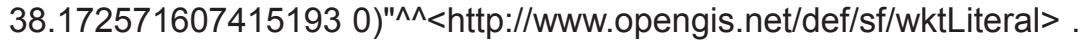




\section{GeoSPARQL}

- Now we will query for the geometries of the counties from the last SPARQL Query

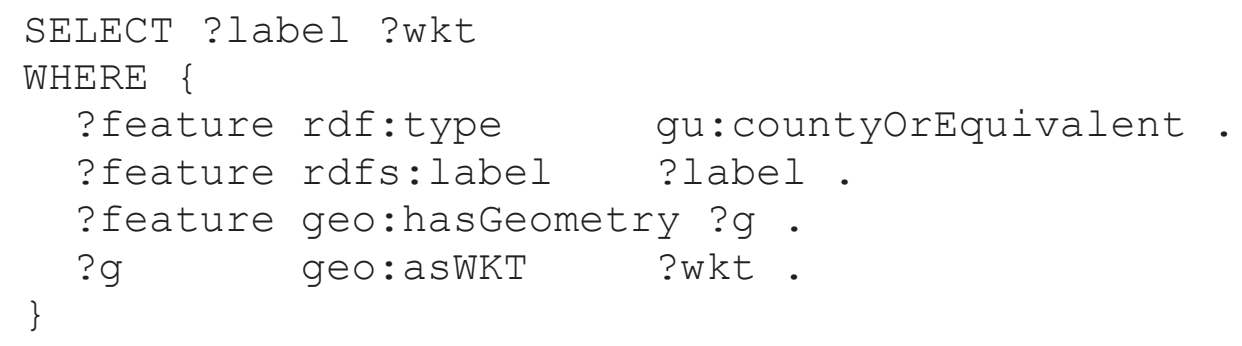

\section{GeoSPARQL}

- Here we have added a new SELECT variable called ?wkt

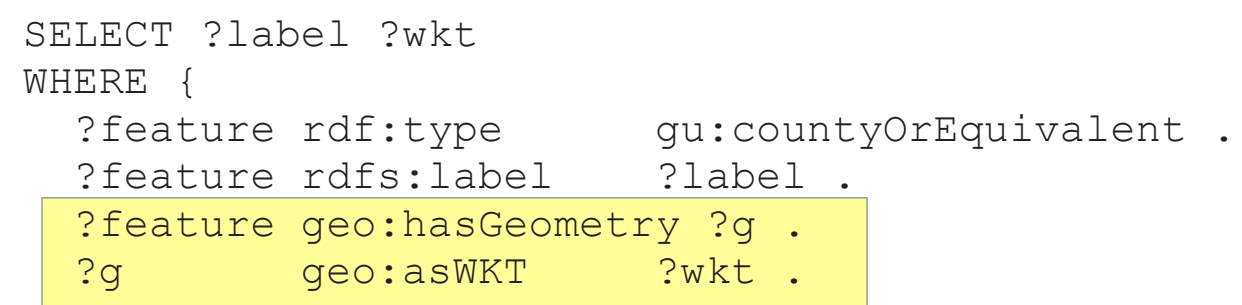

- And we have added two triple patterns 


\section{GeoSPARQL}

- The first triple pattern searches for a geo:Geometry using the geo:hasGeometry predicate

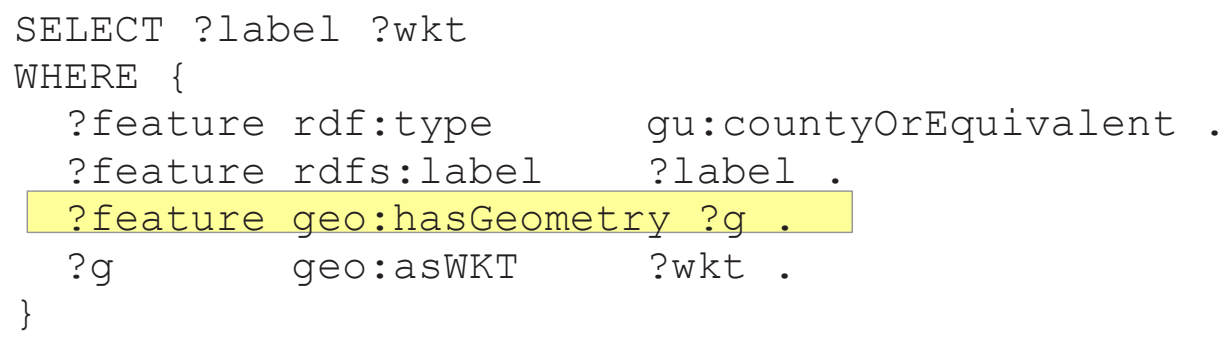

- This geo:Geometry is bound to the variable: ?g

\section{GeoSPARQL}

- The last triple pattern searches for the WKT(a geometry serialization) of the geo:Geometry

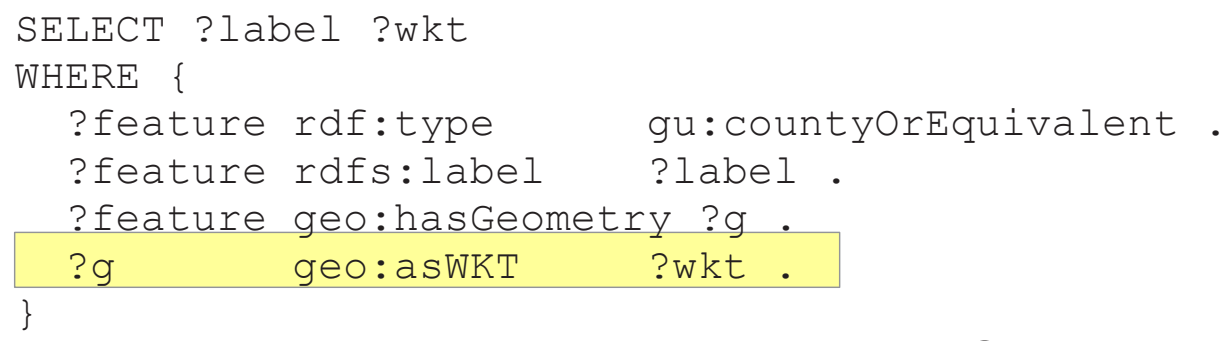

- The WKT is bound to the variable ?wkt 


\section{GeoSPARQL example}

- Open your web browser to the page: http://usgs-ybother.srv.mst.edu/viz/ And click on the "SPARQL Query" button

\section{₹USGS}

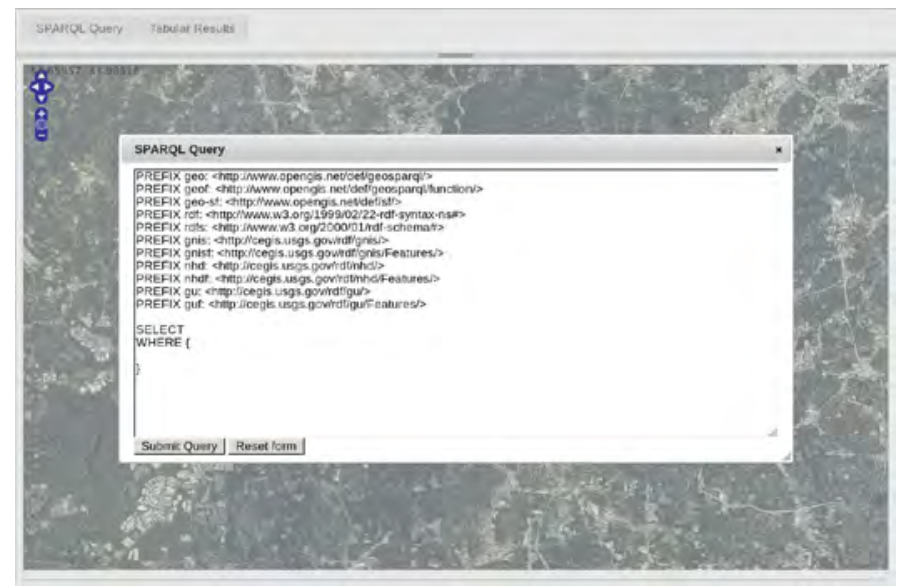

\section{GeoSPARQL example}

- http://usgs-ybother.srv.mst.edu/viz/

- Enter the query:

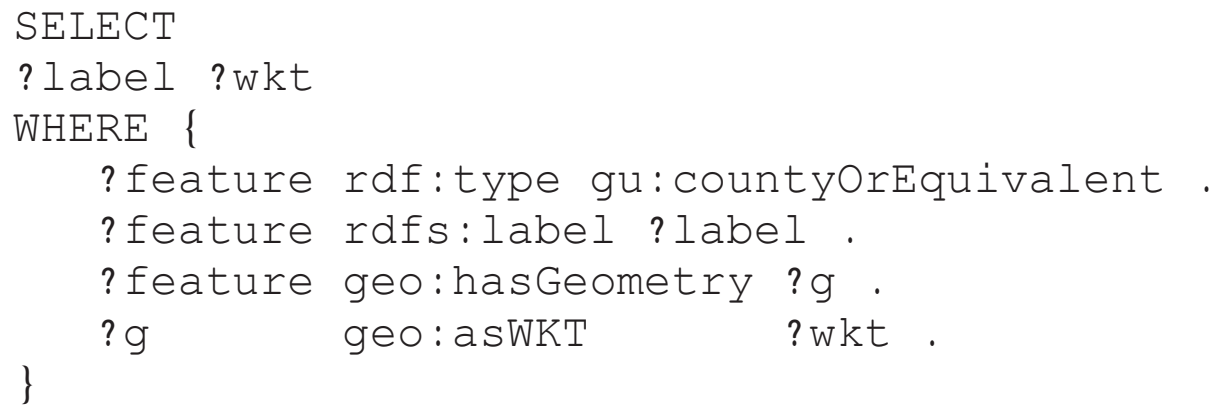




\section{GeoSPARQL Result}

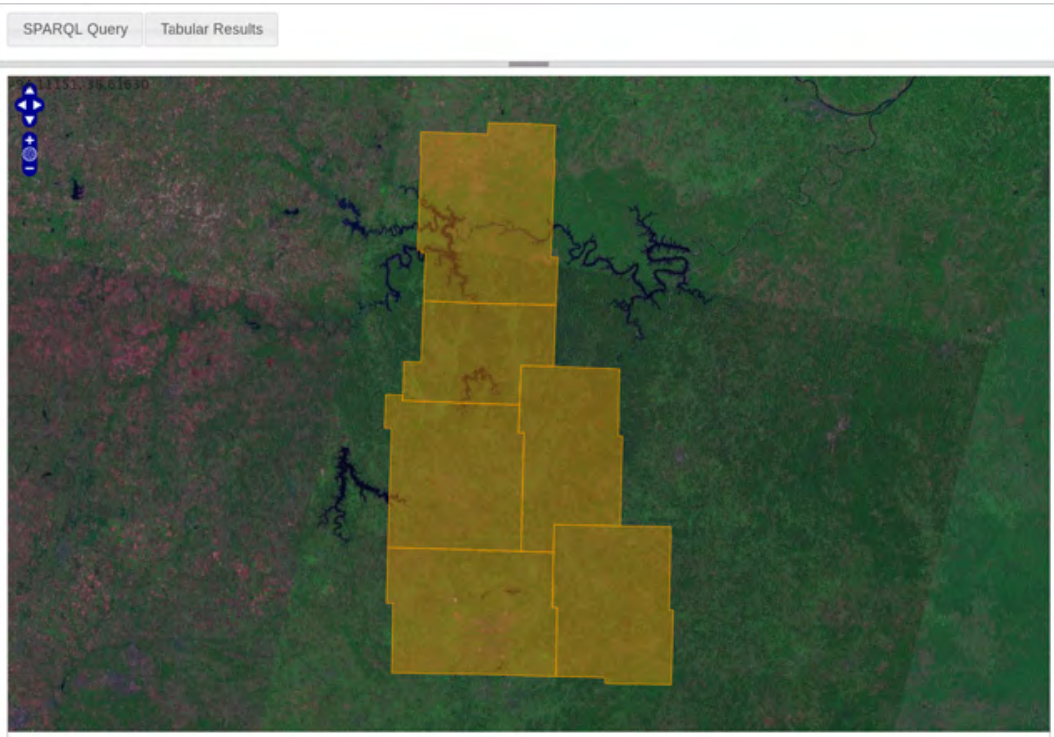

Questions?

\section{FILTER Statements}

- Graph patterns allow us to match RDF terms

- But can we remove matches from the results?

- http://usgs-ybother.srv.mst.edu/viz/

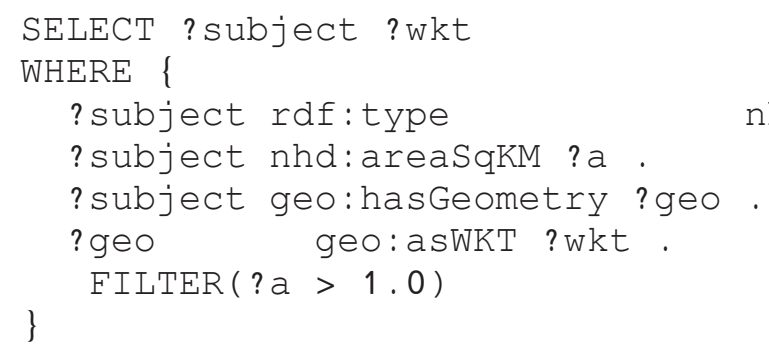

- The FILTER statement restricts matches to those that satisfy the enclosed expression. 
SELECT ? subject ?wkt WHERE \{
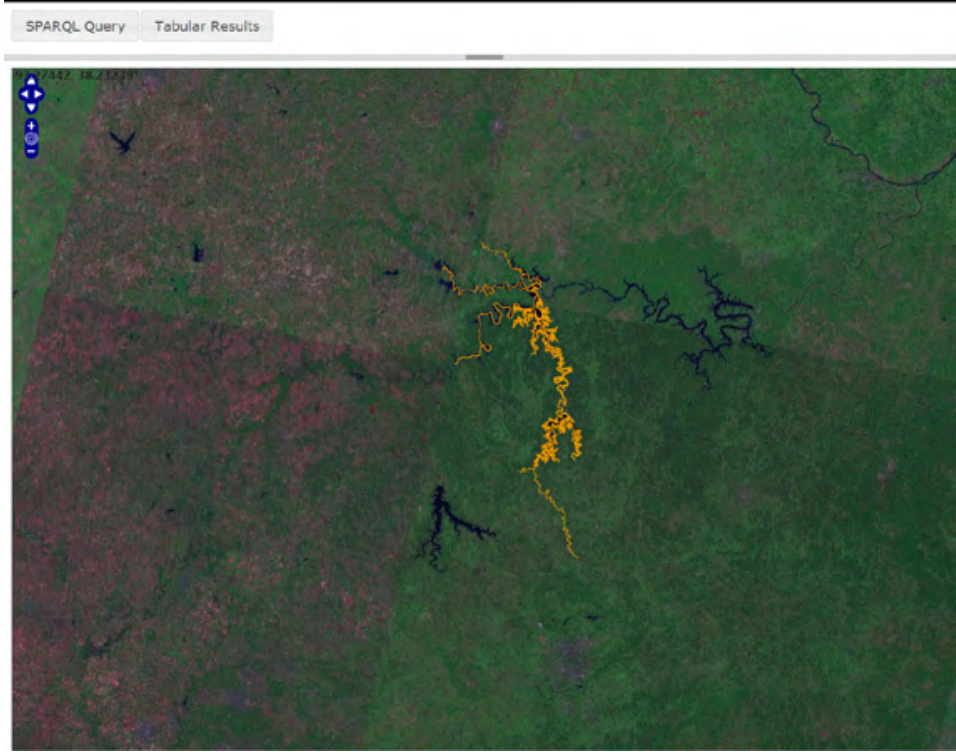

?subject rdf:type nhd:area

?subject nhd:areaSqKM ?a.

? subject geo:hasGeometry ?geo.

?geo geo:asWKT ?wkt.

FILTER(?a > 1.0)

\section{ZUSGS}

\section{FILTER statements}

\section{The GeoSPARQL standard defines vocabulary for topographical relations.}

\section{http://usgs-ybother.srv.mst.edu/viz/}

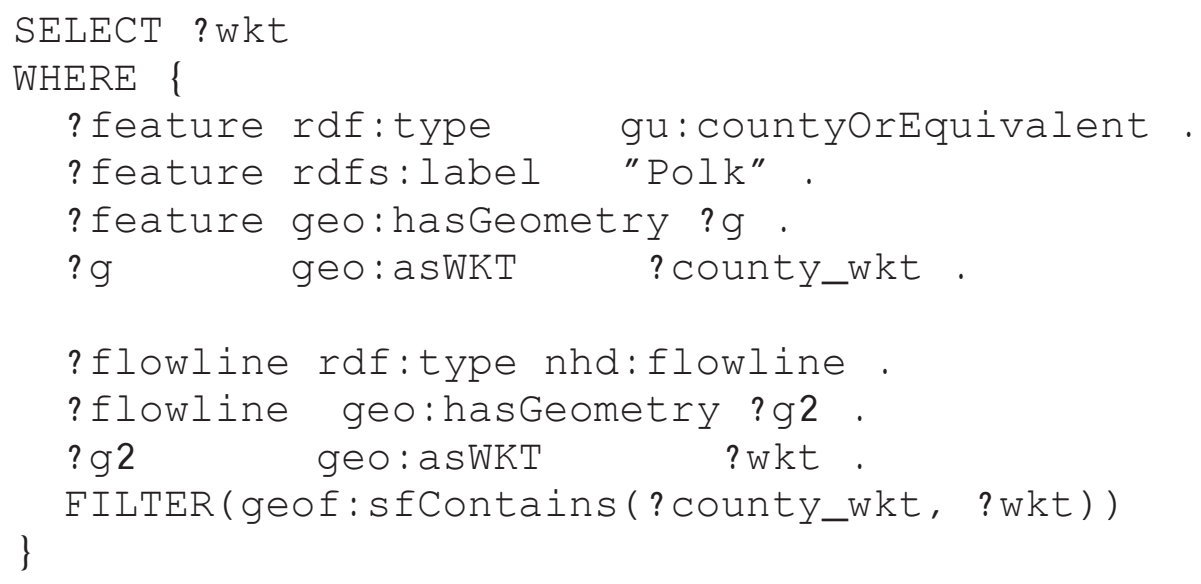




\section{Federated Queries}

With RDF you are not limited to querying the data in your own triplestore

Federated SPARQL queries tell a triplestore to query other servers over a network

\section{‡USGS}

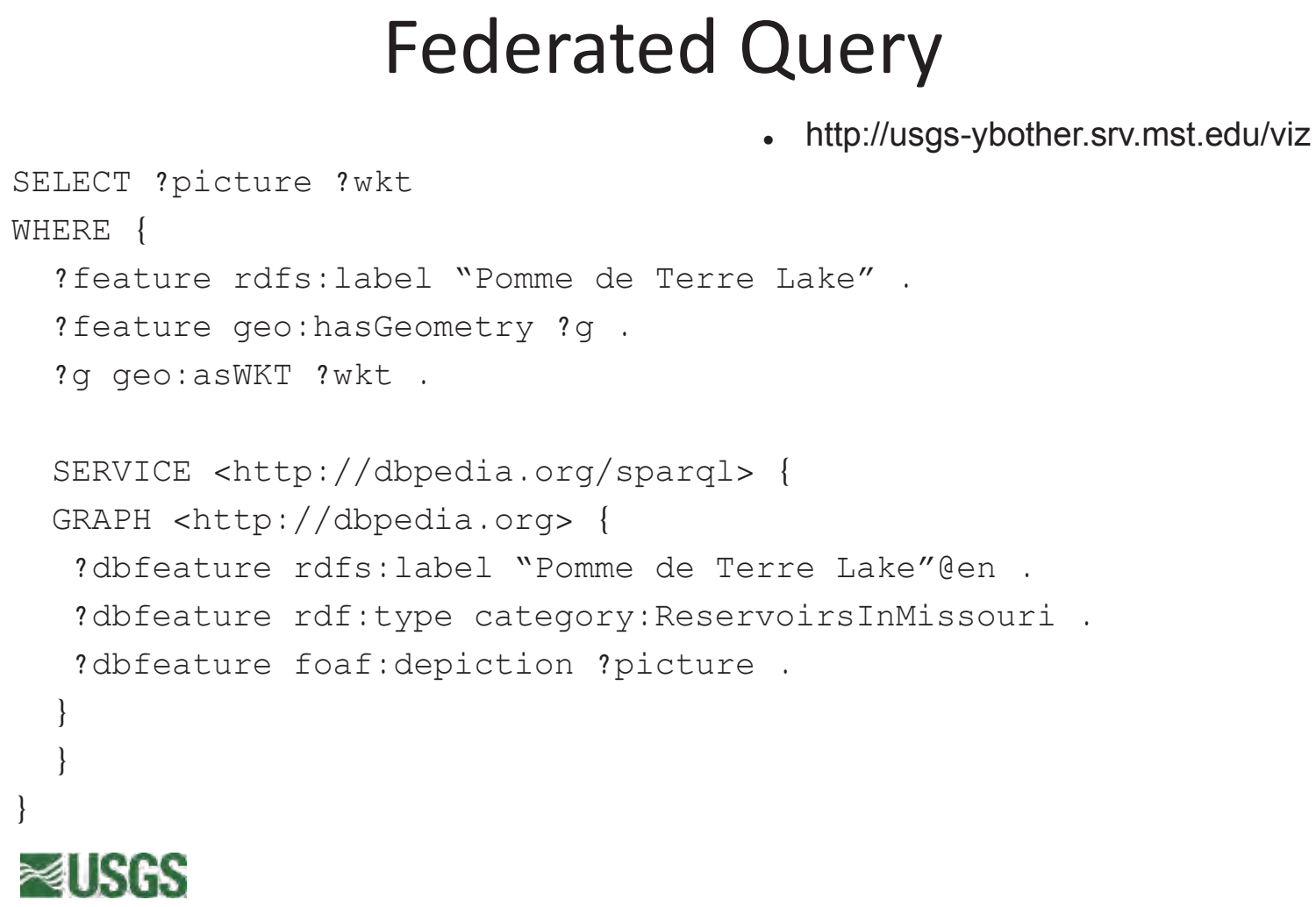

- http://usgs-ybother.srv.mst.edu/viz

SELECT ?picture ?wkt

WHERE \{

?feature rdfs:label "Pomme de Terre Lake" .

?feature geo:hasGeometry ?g .

?g geo:asWKT ?wkt.

SERVICE <http://dbpedia.org/sparql>

GRAPH <http://dbpedia.org> \{

?dbfeature rdfs:label "Pomme de Terre Lake"@en.

?dbfeature rdf:type category:ReservoirsInMissouri .

?dbfeature foaf:depiction ?picture.

\}

\}

\} 


\section{Federated Query}

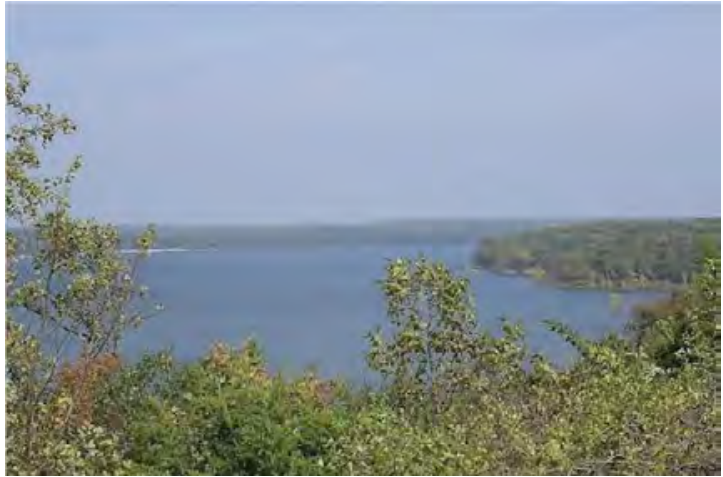

SELECT ?picture ?wkt

WHERE \{

?feature rdfs:label "Pomme de Terre Lake" .

?feature geo:hasGeometry ?g .

?g geo:asWKT ?wkt .

SERVICE <http://dbpedia.org/sparql> \{ GRAPH < http://dbpedia.org> \{

?dbfeature rdfs:label "Pomme de Terre Lake"@en .

?dbfeature rdf:type

category:ReservoirsInMissouri .

?dbfeature foaf:depiction ?picture .

\section{Questions?}




\section{The SOCoP Open Ontology Repository (00R)}

In this session we will demonstrate the use of a geospatial open ontology repository (OOR). The OOR was developed by the Spatial Ontology Community of Practice (SOCoP, www.socop.org)_to help interdisciplinary conversations and collaboration between geoscientists and ontologists. We will illustrate searching and browsing geospatial ontologies (such as GeoSPARQL), how to map terms in different ontologies, how to visualize stored ontologies, and how to add an ontology to the repository. We will discuss plans for federation with other repositories and its interoperation with SPARQL endpoints.

\section{Meteor Crater Ontology}

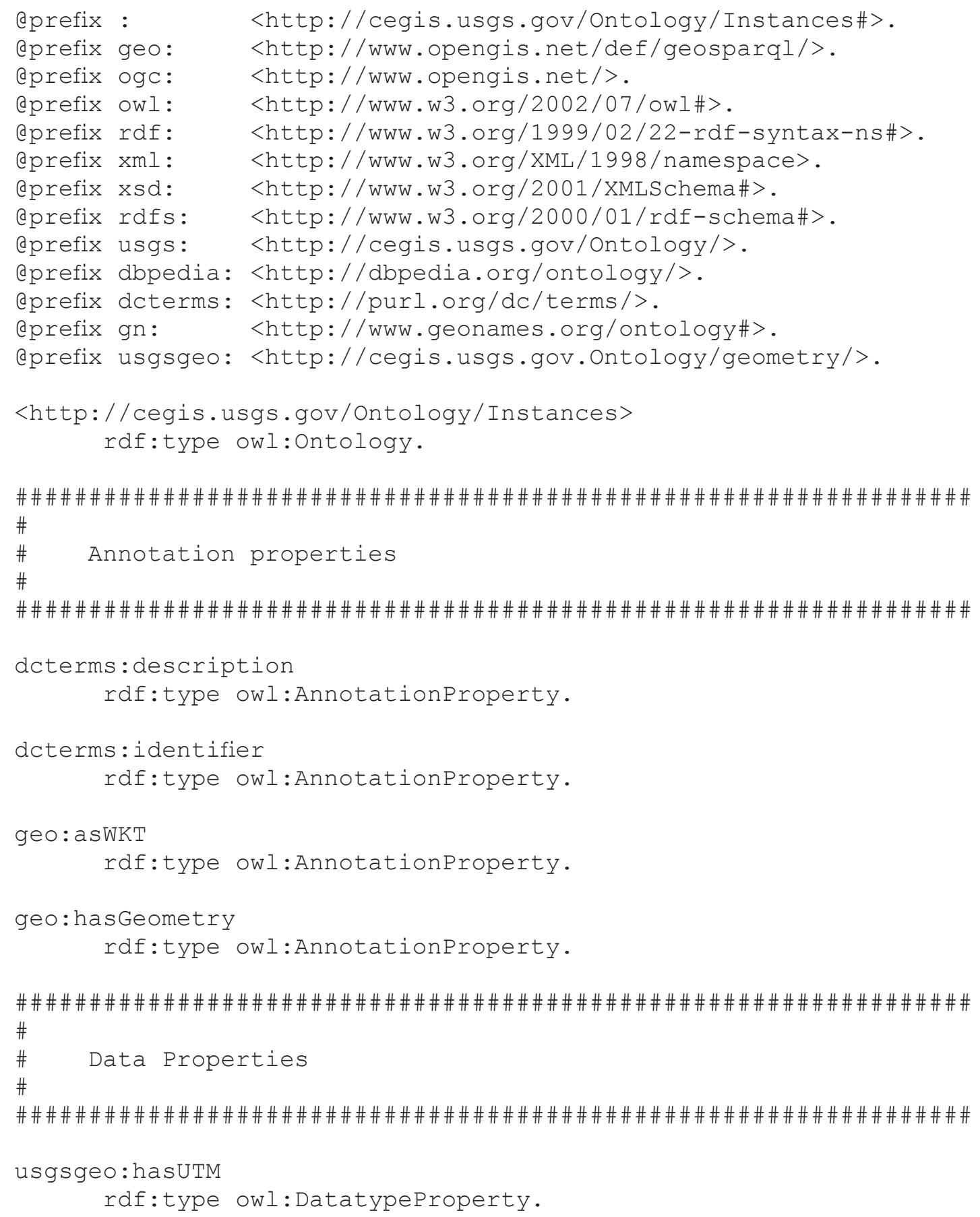




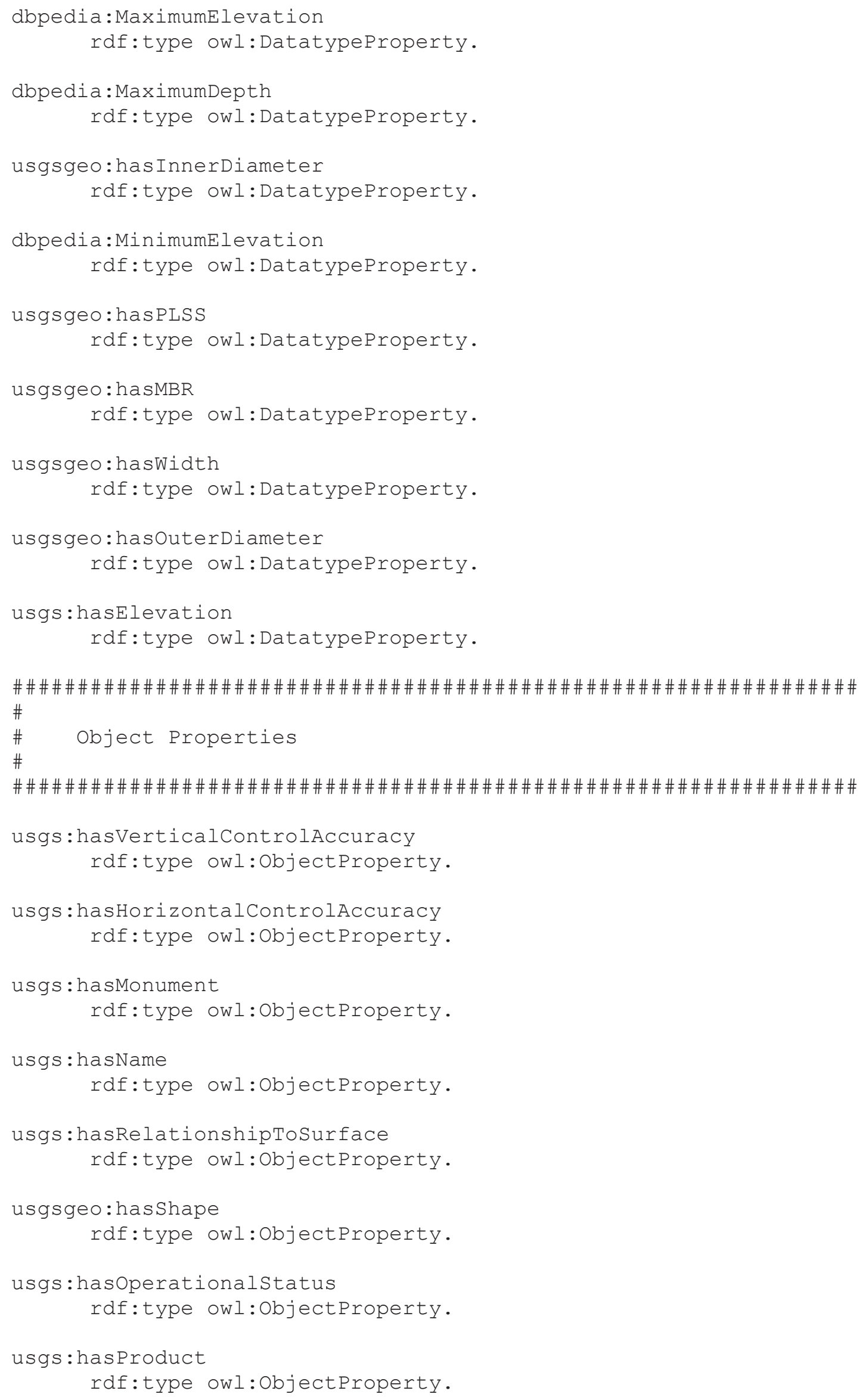




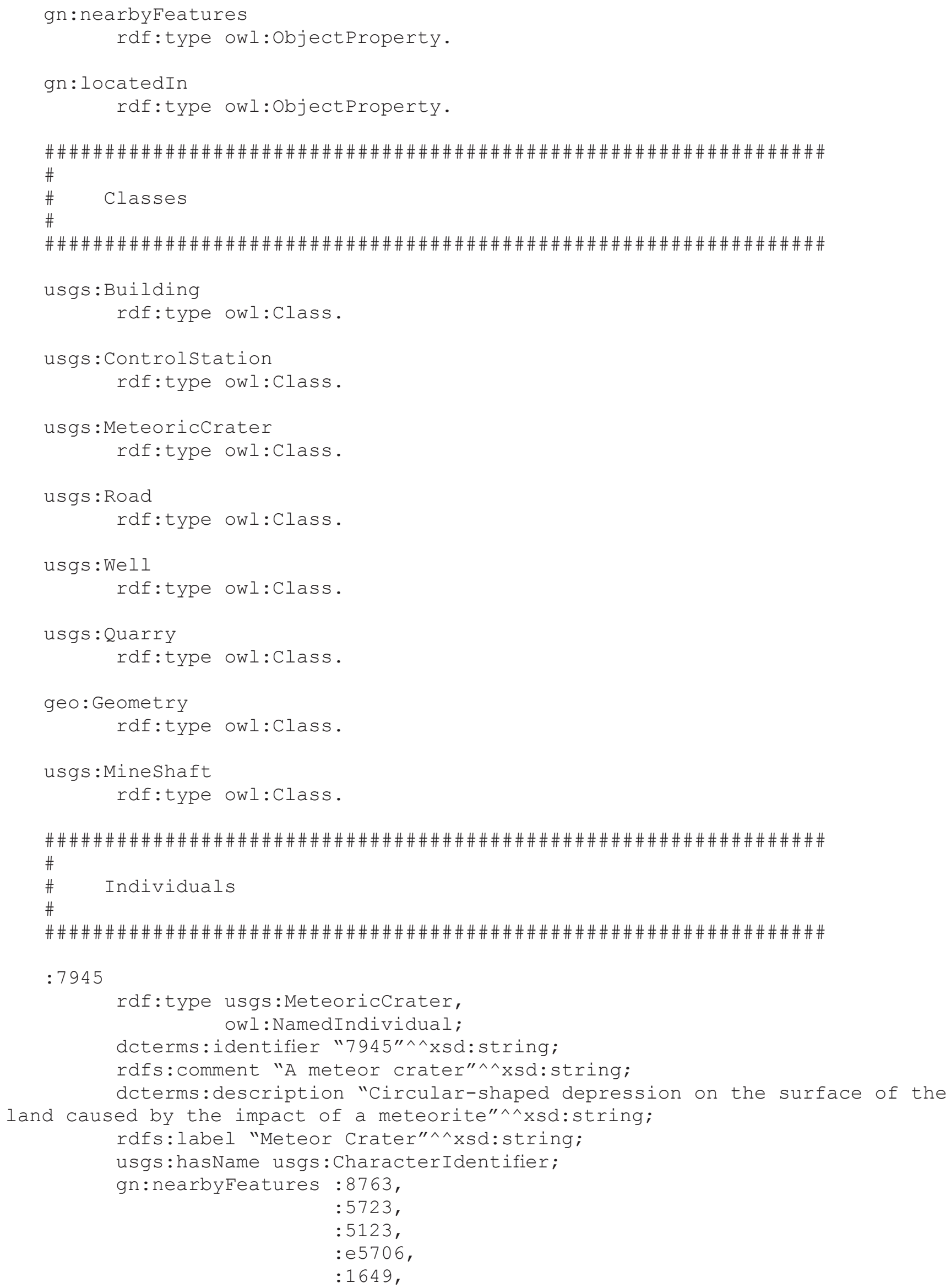




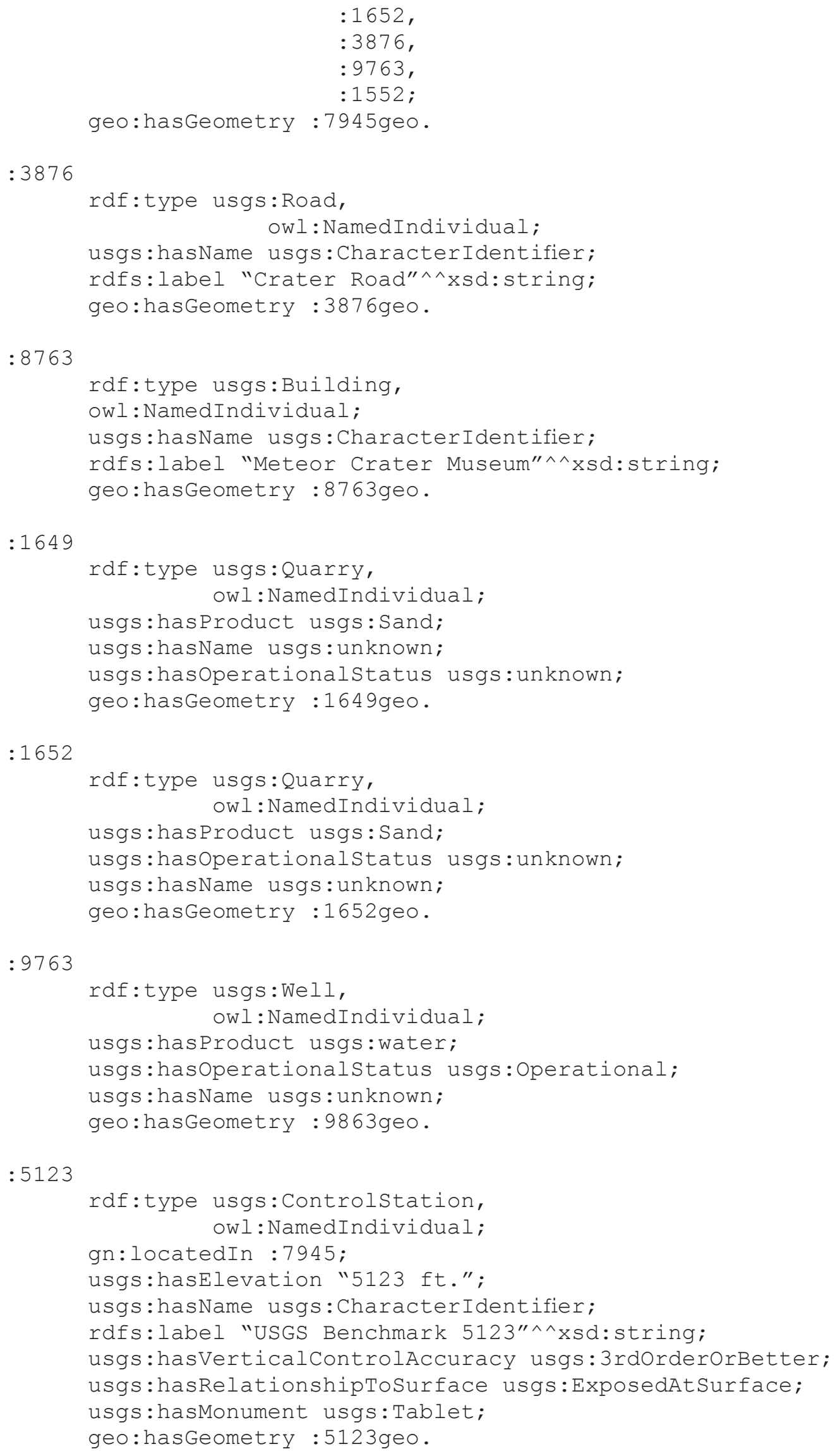


: 5723

rdf:type usgs:Controlstation, owl: NamedIndividual; usgs: hasElevation "5723 ft."; usgs: hasName usgs: CharacterIdentifier; rdfs:label "USGS Benchamrk BM5723"^^xsd:string; usgs: hasHorizontalControlAccuracy usgs:3rdorderorBetter; usgs:hasRelationshipToSurface usgs:ExposedAtSurface; usgs:hasMonument usgs: NoTablet; geo: hasGeometry : 5723geo.

:e5706

rdf:type usgs:Controlstation, owl: NamedIndividual;

usgs:hasElevation "5706 ft.";

usgs:hasName usgs:CharacterIdentifier;

rdfs:label "USGS Benchmark BM East 5706"^^xsd:string;

usgs: hasHorizontalControlAccuracy usgs:3rdorderorBetter;

usgs:hasRelationshipToSurface usgs:ExposedAtSurface;

usgs: hasMonument usgs: NoTablet;

geo:hasGeometry :e5706geo.

: 1497

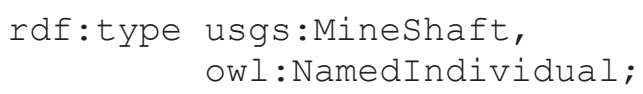

rdf:type usgs:MineShaft, owl: NamedIndividual;

gn:locatedIn :7945;

usgs: hasoperationalstatus usgs: Abandoned;

usgs:hasName usgs:unknown;

geo:hasGeometry :1497geo.

$: 1523$

rdf:type usgs:MineShaft, owl: NamedIndividual;

gn:locatedIn :7945;

usgs:hasOperationalstatus usgs: Abandoned;

usgs: hasName usgs:unknown;

geo:hasGeometry :1523geo.

:1529

rdf:type usgs:MineShaft, owl: NamedIndividual;

gn:locatedIn :7945;

usgs:hasOperationalstatus usgs: Abandoned;

usgs: hasName usgs:unknown;

geo:hasGeometry :1529geo.

$: 1546$

rdf:type usgs:MineShaft,
owl:NamedIndividual;

gn:locatedIn :7945;

usgs: hasoperationalstatus usgs: Abandoned;

usgs:hasName usgs:unknown;

geo:hasGeometry : $1546 \mathrm{geo}$.

$: 1552$

rdf:type usgs:MineShaft, 
owl : NamedIndividual;

usgs: hasOperationalstatus usgs: Abandoned;

usgs: hasName usgs:unknown;

geo:hasGeometry : $1552 \mathrm{geo}$.

: 7945 geo

rdf:type geo:Geometry, owl : NamedIndividual ;

usgsgeo: hasWidth " $0.2 \mathrm{Km}^{\prime}$;

usgsgeo: hasOuterDiameter "1250 m";

dbpedia:MinimumElevation "5123 ft.";

dbpedia:MaximumElevation "5723 ft.";

dbpedia:MaximumDepth "600 ft." ;

usgsgeo:hasInnerDiameter "833 m";

usgsgeo:hasshape usgsgeo:Circular;

usgsgeo:hasUTM "E 497959.94m N 3876020.68m Zone12";

usgsgeo:hasMBR "Max E 489536.79m Min E 497317.62m Max N 3876632.29m Min

N $3875479.58 \mathrm{~m}^{\prime \prime}$;

usgsgeo:hasPLSS "T 19 N, R $121 / 2$ E, Section 13 and 24";

geo:asWKT "POINT -111.02236372362403 35.02684835590344".

: $1649 \mathrm{geo}$

rdf:type geo:Geometry, owl : NamedIndividual;

geo:asWKT "POINT -111.02334 35.02136".

: 1652 geo

rdf:type geo:Geometry,

owl : NamedIndividual;

geo:asWKT "POINT -111.02600 35.02056".

: $3876 \mathrm{geo}$

rdf:type geo:Geometry, owl : NamedIndividual;

geo:asWKT " ".

: 5123 geo

rdf:type geo:Geometry, owl : NamedIndividual;

geo:asWKT "POINT -111.02314 35.02808".

: 5723 geo

rdf:type geo:Geometry, owl: NamedIndividual;

geo:asWKT "POINT -111.02913 35.02945".

:8763geo

rdf:type geo:Geometry, owl : NamedIndividual;

geo:asWKT "POINT -111.02149 35.03270".

: $9863 \mathrm{geo}$

rdf:type geo: Geometry, owl : NamedIndividual;

geo:asWKT "POINT -111.02328 35.03655". 


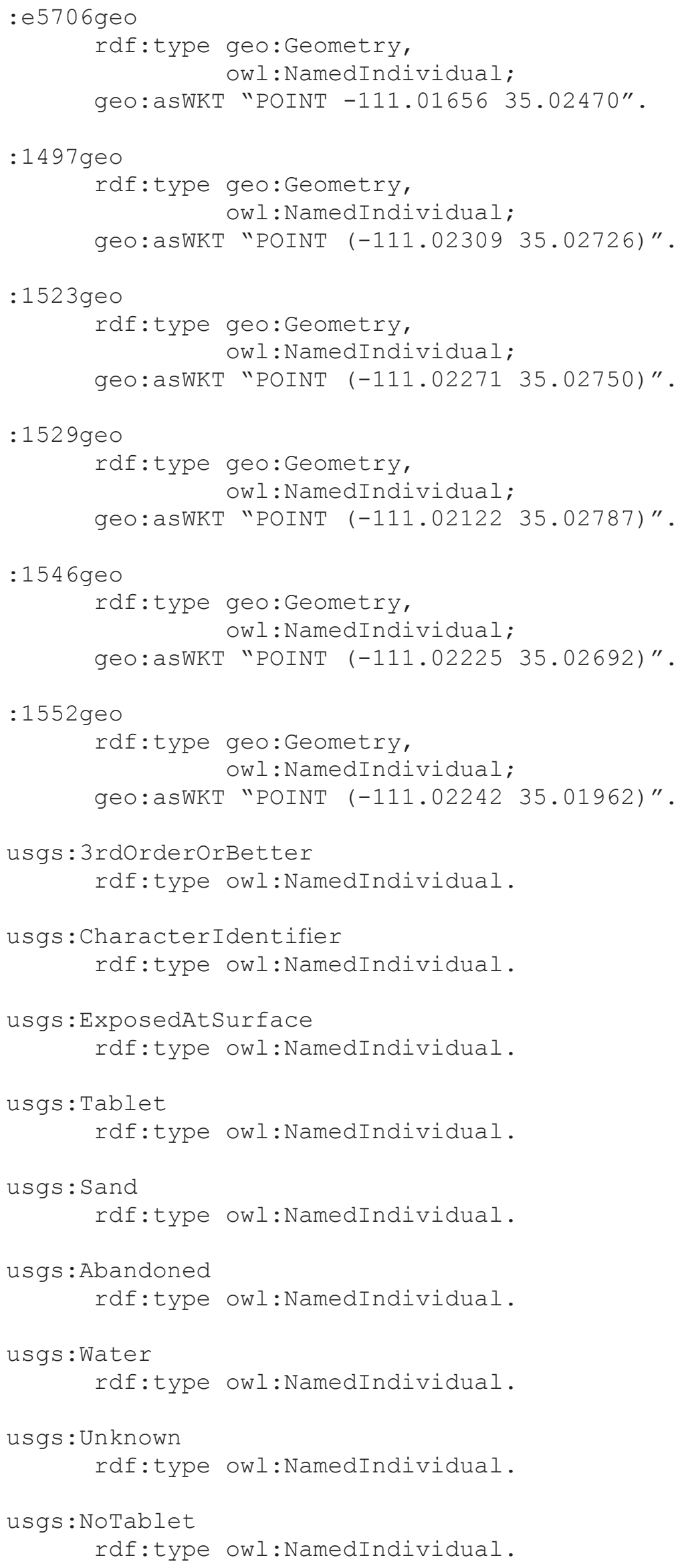




\section{Internet Resources}

Semantic Web. World Wide Web Consortium (W3C).

http://www.w3.org/standards/semanticweb/

\section{Standards and Shared Vocabularies}

Basic Geo (WGS84 lat/long) Vocabulary

http://www.w3.org/2003/01/geo/.

CIDOC - Conceptual Reference Model

http://www.cidoc-crm.org/index.html

DOLCE—Descriptive Ontology for Linguistic and Cognitive Engineering

http://www.loa.istc.cnr.it/DOLCE.html

GeoSPARQL Users Guide 2012

http://ontolog.cim3.net/cgi-bin/wiki.pl?InteropProject/Geosparql_USER_GUIDE_2012

Glossary of Semantic Web Terms

https://wiki.base22.com/display/btg/Glossary+of+Semantic+Web+Terms\#GlossaryofSemanticWebTerms-T

Publishing Vocabularies

http://www.w3.org/TR/swbp-vocab-pub/

Resource Description Framework (RDF) Primer

http://www.w3.org/TR/2004/REC-rdf-primer-20040210/

SPARQL Protocol and RDF Query Language (SPARQL)

http://www.w3.org/TR/rdf-sparql-query/.

Turtle-Terse RDF Triple Language

http://www.w3.org/TR/2011/WD-turtle-20110809/

WordNet—WordNet, A lexical database for English. Princeton University.

http://wordnet.princeton.edu/.

\section{Software and Technology Products}

AllegroGraph RDFStore

http://www.franz.com/agraph/

Jena

http://openjena.org/wiki/TDB

Oracle

http://www.oracle.com/technetwork/database/options/semantic-tech/index.html

Parliament

http://parliament.semwebcentral.org/

Protégé Ontology Editor

http://protege.stanford.edu/ 
Semantic Web Development Tools

http://www.w3.org/2001/sw/wiki/Tools

SemWebCentral

http://www.semwebcentral.org/

TopBraid Composer

http://www.topquadrant.com/products/TB_Composer.html

\section{Ontologies and Linked Data}

OpenCyc

http://sw.opencyc.org/

DBpedia

http://dbpedia.org/About

e-Government

http://oegov.org/

Freebase

http://www.freebase.com/

Linked Open Data Initiative

http://linkeddata.org/

LinkedGeoData

http://linkedgeodata.org/About

NeoGeo Vocabulary

http://geovocab.org/doc/survey.html

OntologyDesignPatterns.org

http://ontologydesignpatterns.org/wiki/Main_Page.

Open Ontology Repository Initiative

http://OpenOntologyRepository.org

Semantic MediaWiki

http://mapping.referata.com/wiki/Semantic_Maps

Semantic Web

http://semanticweb.org/wiki/Main_Page

Semantic Web for Earth and Environmental Terminology (SWEET) Ontologies

http://sweet.jpl.nasa.gov/

U.S. Geological Survey Triple Data

http://usgs-ybother.srv.mst.edu:8890/parliament

\section{Online Tutorials}

Information Semantics 101: Semantics, Semantic Models, Ontologies, Knowledge Representation, and the Semantic Web http://c4i.gmu.edu/OIC09/workshop.php 
Introduction to Ontologies and Semantic Web [On-line]

http://www.obitko.com/tutorials/ontologies-semantic-web/

Introduction to Ontologies and Semantic Technologies

http://stids.c4i.gmu.edu/STIDS2011/agenda2011.php

SPARQL By Example, A Tutorial

http://www.cambridgesemantics.com/semantic-university/sparql-by-example

\section{Ontology Communities, Professional Organizations, and Workshop Events}

International Association for Ontology and its Applications (IOAO)

http://www.iaoa.org/

The 11th International Semantic Web Conference

http://iswc2012.semanticweb.org/

Terra Cognita 2011 Workshop

http://asio.bbn.com/terracognita2011

Semantic Technology for Intelligence, Defense, and Security (STIDS)

http://stids.c4i.gmu.edu/

Federation of Earth Science Information Partners (ESIP) Semantic Web Cluster

http://wiki.esipfed.org/index.php/Semantic_Web

Spatial Ontology Community of Practice (SOCoP)

http://www.socop.org/

Ontolog collaborative work environment

http://ontolog.cim3.net/

\section{Research Groups and Programs of Study}

GeoLinkedData.es, Ontology Engineering Group

http://geo.linkeddata.es/web/guest

Laboratory for Applied Ontology

http://www.loa.istc.cnr.it/

Tetherless World Constellation

http://tw.rpi.edu/web/TWC

Muenster Semantic Interoperability Lab

http://musil.uni-muenster.del

\section{Blogs}

Data.gov/semantic

http://www.data.gov/communities/node/116/blogs

John Goodwin

http://www.johngoodwin.me.uk/ 


\section{Suggested Literature}

\section{Semantic and Geospatial Semantic Web}

Berners-Lee, T., Hendler, J., and Lassila, O., 2001, The Semantic Web: Scientific American, May 2001, p. 35-43.

Egenhofer, M.J., 2002, Toward the Semantic Geospatial Web, in 10th ACM International Symposium on Advances in Geographic Information Systems (ACM-GIS), November 8-9, 2002. McLean, Va., p. 1-4.

Hitzler, P., and van Harmelen, F., 2010, A Reasonable Semantic Web. Semantic Web_-Interoperability, Usability, Applicability: Semantic Web, IOS Press, p. 39-44. (Also available at http://dx.doi.org/ 10.3233/SW-2010-0010.)

Kuhn, W., 2005, Geospatial Semantics - Why, of what, and how? Journal on Data Semantics III, v. 3534, p. 1-24.

Noy, N.F., and McGuinness, D.L., 2001, Ontology Development 101-A guide to creating your first ontology: Stanford Knowledge Systems Laboratory Technical Report KSL-01-05 and Stanford Medical Informatics Technical Report SMI-2001-0880, Stanford University.

\section{Geospatial Semantics and Ontology}

Arpinar, I.B., Sheth, A., Ramakrishnan, C., Usery, E.L., Azami, M., and Kwan, M., 2004, Geospatial ontology development and semantic analytics in Wilson, J.P., and Forthingham, A.S., eds., Handbook of Geographic Information Science, Blackwell Publishing.

Corcho, O., Fernández-López, M., and Gómez-Pérez, A., 2003, Methodologies, tools and languages for building ontologies. Where is their meeting point? Data and Knowledge Engineering v. 46, p. 41-64.

Frank, A.U., 2001, Tiers of ontology and consistency constraints in geographic information systems: International Journal of Geographic Information Science, v. 15, no. 7, p. 667-678.

Gangemi, A., and Presutti, V., 2009, Ontology design patterns, in Staab, S., and Studer, R., eds., Handbook of Ontologies (2d ed.): Berlin, Springer.

Kokla, M., and Kavouras, M., 2001, Fusion of top-level and geographical domain ontologies based on context formation and complementarity: International Journal of Geographic Information Science, v. 15, no. 7, p. 679-687.

Kuhn, W., 2001, Ontologies in support of activities in geographical space: International Journal of Geographic Information Systems, v.15, no. 7, p. 613-631.

Lutz, M., and Klien, E., 2006, Ontology-based retrieval of geographic information: International Journal of Geographical Information Science, v. 20, no. 3, p.233-260.

Perry, M., Sheth, A., Arpinar, I.B., and Hakimpour, F., 2009, Geospatial and temporal semantic analytics, in, Karimi, H.A., ed., Handbook of Research on Geoinformatics: Hershey, Pa., Information Science Reference, p.161-170.

Schuurman, N., and Leszczynski, A., 2006, Ontology-based metadata: Transactions in GIS, v. 11, p. 709-26.

Sen, S., 2008, User of Affordances in Geospatial Ontologies, in Proceedings of the 2006 International Conference on Towards affordance-based robot control, no. 4760, Springer-Verlag, p. 122-139.

Staab, S., and Studer, R., eds., 2004, The Handbook on Ontologies: Berlin, Springer-Verlag, 660 p. 


\section{Taxomony, Mereotopology and Other Relations}

Behr, R,. and Schneider, M., 2001, Topological relations of complex points and complex regions, in International Conference on Conceptual Modeling, 20th, Yokohama, Japan, 2001, Lecture Notes in Computer Science 2224, Berlin, Springer-Verlag p. 56-69.

Casati, R., and Varzi, A., 1999, Parts and places, the structures of spatial representation: Cambridge, Mass., Massachusetts Institute of Technology Press, $238 \mathrm{p}$.

Green, R., Bean, C.A., and Myaeng, S.H., eds., 2002,The semantics of relationships-An interdisciplinary perspective: Dordrecht, Kluwer, 223 p.

Perry, M., and Herring, J., eds., 2012, GeoSPARQL_A geographic query language for RDF data: Open Geospatial Consortium OGC 11-052r4. (Also available at http://www.w3.org/2011/02/GeoSPARQL.pdf.)

Welty, C., and Guarino, N., 2001, Supporting ontological analysis of taxonomic relations: Data and Knowledge Engineering, v. 39, p. 51-74.

\section{Linked Data and Social Networking}

Cucchiarelli, A., and D'Antonio, F., and Velardi, P., 2011, Semantically interconnected social networks: Social Network Analysis and Mining, Springer, p. 69-95. (Also available at $h t t p: / / w w w . s p r i n g e r l i n k . c o m / c o n t e n t / p 8 p 809 h 04527 n 88 q /$.

Gruber T., 2007, Ontology of folksonomy-A Mash-up of apples and oranges: International Journal on Semantic Web and Information Systems, v. 3, no. 1, 11 p. (Also available at http://tomgruber.org/writing/ontology-of-folksonomy.htm.)

Hahmann, S., and Burghardt, D., 2010, Connecting LinkedGeoData and Geonames in the spatial semantic Web, in Proceedings of the 6th International GIScience Conference, Zurich, Switzerland, 2010. (Also available at http://kartographie.geo. tu-dresden.de/aigaion/attachments/Hahmann_Burghardt_LinkedGeoData_Geonames.pdf-c9d454e361d4e3188da338f4 ffc66864.pdf.)

\section{Application Engineering}

Allemang, D., and Hendler, J., 2008, Semantic Web for the working ontologist, effective modeling in RDFS and OWL: Burlington, Mass., Morgan Kaufmann, 330 p.

Dago, E., Blomqvist, E., Gangemi, A., Montiel, E., Nikitina, N., Presutti, V., and Villazon-Terrazas, B., 2005, Pattern based ontology design - Methodology and software support-NeOn—Lifecycle Support for Networked Ontologies: Integrated Project (IST-2005-027595). (Also available at http://www.neon-project.org/nw/images/5/5c/NeOn_2010_D252.pdf.)

Guarino, N., and Welty, C., 2002, Evaluating ontological decisions with OntoClean: Communications of the ACM, v. 45, no. 2, p. 61-65.

Pulido, J.R.G., Ruiz, M.A.G., Herrera, R., Cabello, E., Legrand, S., and Elliman, D., 2006, Ontology languages for the semantic web-A never completely updated review: Knowledge-Based Systems, v. 19, no. 7, p. 489-497.

\section{Geography, GIScience, and Geolnformatics}

Agarwal, P., 2005, Ontological considerations in GIScience: International Journal of Geographical Information Science, v. 19 , no. 5, p. 501-536.

Couclelis, H., 2010, Ontologies of geographic information: International Journal of Geographical Information Science, v. 24, no. 12 , p. $1,785-1,809$.

Fonseca, F., Egenhofer, M., Davis, C. and Camara, G., 2002, Semantic granularity in ontology-driven geographic information systems: Annals of Mathematics and Artificial Intelligence, v. 36, p. 121-151. 
Frank, A.U., 2003, Ontology for spatio-temporal databases, chap. 2 of Spatiotemporal databases-The Chorochronos Approach: Koubarakis, M. , Sellis, T.K. , Frank, A.U., Grumbach, S., Güting, R.H., Jenson, C.S., Lorentzos, N., Manolopoulos, Y., Nardelli, E., Pernici, B., Schek, H-J., Scholl, M., Theodoulidis, B., and Tryfona, N., eds., Springer, ser. Lecture Notes in Computer Science, v. 2520, 352 p.

Painho, M., Curvelo, P., and Jovani, I., 2007, An ontological-based approach to Geographic Information System curricula design: The European Information Society, Lecture Notes in Geoinformation and Cartography, part 1, p. 15-34.

Schuurman, N., 2006, Formalization Matters - Critical GIS and Ontology Research: Annals of the Association of American Geographers, v. 94, no. 4, p. 726-739.

Zhou, N., 2011, Ontological and semantic technologies for geospatial portals in Zhao, P., and Di, L., eds., Geospatial Web Services-Advances in Information Interoperability: Hershey, Pa., IGI Global, p. 227-243.

\section{Land Cover}

Ahlqvist, O., 2008, Extending post-classification change detection using semantic similarity metrics to overcome class heterogeneity-A study of 1992 and 2001 U.S. National Land Cover Database changes: Remote Sensing of Environment, v.112, p. $1,226-1,241$.

Feng, C., and Flewelling, D. M., 2004, Assessment of semantic similarity between land use/landcover classification systems: Computers, Environment and Urban Systems, v. 28, issue 3, p. 229-246.

\section{Ecology and Environmental Monitoring}

Bittner, T., 2007, From top-level to domain ontologies-Ecosystem classifications as a case study, in Conference on Spatial Information Theory, 9th, Melbourne, Australia, 2007, Cognitive and Computational Foundations of Geographic Information Science, p. 61-77.

Fonseca, F., Martin, J., and Rodrigues, A., 2002, From geo- to eco-ontologies, in Egenhofer, M.J., and Mark, D.M., eds., Geographic information science: Springer.

Pundt, H., and Bishr, Y., 2002, Domain ontologies for data sharing-An example from environmental monitoring using field GIS: Computer and Geosciences, v. 28, issue 1, p. 95-102.

Sorokine, A., Bittner, T., and Renscher, C., 2006, Ontological investigation of ecosystem hierarchies and formal theory for multiscale ecosystem classifications: Geoinformaticea, v. 10, no. 3, p. 313-335.

\section{Terrain}

Brodaric, B., 2007, Geo-pragmatics for the geospatial semantic web: Transactions in GIS, v. 11, issue 3, p. 453-477

Brodaric, B., and Gahegan, M., 2007, Experiments to examine the situated nature of geoscientific concepts: Spatial Cognition and Computation, v. 7, issue 1, p. 61-95.

Mark, D.M., and Smith, B., 2004, A science of topography_From qualitative ontology to digital representations, in Bishop, M.P., and Shroder, J.F., Jr., eds., Geographic information science and mountain geomorphology: Chichester, United Kingdom, Praxis Publishing, p. 75-100.

Sinha, A. K., Malik, Z., Rezgui, A., Barnes, C.G., Lin, K., Heiken, G., Thomas, W.A., Gundersen, L.C., Raskin, R., Jackson, I., Fox, P., McGuinness, D., Seber, D., and Zimmerman, H., 2010, Geoinformatics-Transforming data to knowledge for geosciences: GSA Today, v. 20, no. 12, p. 4-10.

Smith, B., and Mark, D., 2003, Do mountains exist?-Towards an ontology of landforms: Environment and Planning, v. 30, issue 3, p. 411-427. 


\section{Ontology of Rasters and Images}

Bittner, T., and Winter, S., 1999, On ontology in image analysis in Integrated Spatial Databases, in Agouris, P., and Stefanidis, A., eds., Lecture Notes in Computer Science, v. 1737, p. 168-191

Camara, G., Egenhofer, M., Fonseca, F., and Monteiro, A.M.V., 2001, What's in an Image? in Montello, D.R., ed., Spatial Information Theory-Foundations of geographic information science: Berlin, Germany, Springer-Verlag, Lecture Notes in Computer Science, v. 2205, p. 474-488.

Hornsby, K., 2004, Retrieving event-based semantics from images in Conference on Multimedia Software Engineering, 6th, Miami, Fla., Los Alamitos, Calif., 2004, Institute of Electrical and Electronics Engineers, p. 529-536.

Liu, Y., Lin, Y., Qin, S., Zhang, Y., and Wu, L., 2005, Research on GSQL Extension Supporting Raster Data, Journal of Image and Graphics, accessed May 31, 2011, at http://en.cnki.com.cn/Article_en/CJFDTOTAL-ZGTB20050100J.htm

Quintero, R., Torres, M., Moreno, M., and Guzman, G., 2009, Towards a semantic representation of raster spatial data in International Conference on Geospatial Semantics, 3d, Mexico City, Mexico, 2009, Lecture Notes in Computer Science 5892, Berlin, Springer-Verlag, p. 63-82.

Zheng, B., Huang, L., and Zinhai, L., 2009, Ontology for the cell-based geographic information in Liu, Y., and Tang, X., eds., International Symposium on Spatial Analysis, Spatial-Temporal Data Modeling, and Data Mining: Proc. Of SPIE, v. 7492.

\section{Similarity and Interoperability}

Bish, Y., 1998, Overcoming the semantic and other barriers to GIS interoperability: International Journal of Geographical Information Science, v. 12, no. 4, p. 299-314.

Bittner, T., Donnelly, M., Smith, B., 2006, A spatio-temporal ontology for geographic information integration: International Journal of Geographical Information Science, v. 23, issue 6, p. 765-798

Cruz, I.F., and Sunna, W., 2008, Structural alignment methods with applications to geospatial ontologies. Transactions in GIS, v. 12 , issue 6 , p. 683-711.

Fonseca, F., David, C., and Câmara, G., 2003, Bridging ontologies and conceptual schemas in geographic information integration: Geoinformatica v. 7, issue 4, p. 355-378.

Fonseca, F., Egenhofer, M., Agouris, P., and Câmara, G., 2002, Using ontologies for integrated geographic information systems: Transactions in GIS, v. 6, no. 3, p. 231-257.

Kavouras, M., and Kokla, M., 2008, Theories of geographic concepts-Ontological approaches to semantic integration: Boca Raton, Fla., CRC Press, 319 p.

Kokla, M., and Kavouras, M., 2005, Semantic information in geo-ontologies-Extraction, comparison, and reconciliation: Journal on data semantics, v. 3, p. 125-142.

Rodríguez, M. A., Egenhofer, M.J., and Rugg, R.D., 1999, Assessing semantic similarities among geospatial feature class definitions in Vckovski, A., Brassel, K., and Schek, H.J., eds., Interoperating geographic information systems, Interop '99, Zurich, Switzerland: Springer-Verlag, Lecture Notes in Computer Science, v. 1580, p. 189-202.

Schwering, A., 2008, Approaches to semantic similarity measurement for geo-spatial data: Transactions in GIS, v. 12, issue 1, p. 5-29.

Uitermark, H.T., van Oosterom, P.J.M., Mars, N.J.I., and Molenaar, M., 2005, Ontology-based integration of topographic data sets: International Journal of Applied Earth Observation and Geoinformation, v. 7, p. 97-106.

\section{Logic and Knowledge Representation and Reasoning}

Bouquet, P., Ghidini, C., Giunchiglia, F., and Blanzieri, E., 2003, Theories and uses of context in knowledge representation and reasoning: Journal of Pragmatics, v. 35, issue 3, p. 455-484. 
Brachman, R.J., and Levesque, H.J., 2004, Knowledge representation and reasoning: San Francisco, Calif., Morgan Kaufman, $381 \mathrm{p}$.

Zhang, C., Zhao, T., Li, W., and Osleeb, J.P., 2010, Towards logic-based geospatial feature discovery and integration using web feature service and geospatial semantic web: International Journal of Geographical Information Science, v. 24, issue 6, p. 903-923.

Sowa, J.F., 2000, Knowledge representation-Logical, philosophical, and computational foundations: Brooks/Cole, 608 p.

\section{USGS Resources}

Guptill, S., ed., 1990, An enhanced digital line graph design: U.S. Geological Survey Circular 1048

Spatial Data Transfer Standard Technical Review Board, 1997, Spatial Data Transfer Standard part 2-Spatial features: Federal Geographic Data Committee, (Also available at http://mcmcweb.er.usgs.gov/sdts/SDTS_standard_nov97/p2anxa. html\#342523.)

U.S. Board on Geographic Names, 2010b, Geographic Names Information System (GNIS): U.S. Geological Survey, accessed on DATE, at $h$ ttp://geonames.usgs.gov/pls/gnispublic/f?p=gnispq:8:1829334408278873

U.S. Geological Survey, Digital line graph standards, (Also available at http://nationalmap.gov/standards/dlgstds.html.)

Varanka, D., 2009a, Landscape features, technology codes, and semantics in U.S. National Topographic Mapping Databases, in The International Conference on Advanced Geographic Information Systems and Web Services (GEOWS), Cancun, Mexico, 2009, Geographic Information Systems and Web Services.

Varanka, D., 2009b, A topographic feature taxonomy for a U.S. National topographic mapping ontology, in International Cartographic Conference, 24th, Santiago, Chile, 2009, International Cartographic Association, [CD-ROM publication].

Varanka, D., 2011, Ontology patterns for complex topographic feature types: Cartography and Geographic Information Science, v. 38 , no. 2 , p. $126-136$.

Varanka, D.E., Carter, J.J., Shoberg, T., and Usery, E.L., 2011, Topographic mapping data semantics through data conversion and enhancement, in Sheth, Amit, and Ashish, Naveen, eds., Geospatial semantics and the semantic web-Foundations, algorithms, and applications: Springer, Semantic Web and Beyond, v. 12, p. 145-162.

Usery, E.L., and Varanka, D.E., 2012, Design and development of linked data for The National Map: The Semantic Web Journal. Also available at: http://www.semantic-web-journal.net/content/design-and-development-linked-data-national-map.

\section{Edited Journal Issues and Proceedings from Scholarly Meetings}

Crampton, J., ed., 2010, Ontological issues for The National Map: Cartographica: The International Journal for Geographic Information and Visualization, v. 45, no. 2, p. 103-104.

Hitzler, P., and Janowicz, K., eds., 2012, Semantic web-Interoperability, usability, applicability, v. 3, no. 1, IOS Press.

Janowicz, K., Raubal, M., and Levashkin, S., eds., 2009, GeoSpatial semantics, in International Conference, 3d, Mexico City, Mexico, 2009, Lecture Notes in Computer Science 5892, Berlin, Springer-Verlag.

Kuhn, W., Worboys, M., and Timpf, S., eds., 2009, Spatial information theory-Foundations of geographic information science in Central organization for statistics and information technology international conference, Aber Wrac'h, France, 2009, Lecture Notes in Computer Science 2825, Berlin, Springer-Verlag.

Wiegand, N., Berg-Cross, G., and Varanka, D.E., eds., 2011, First ACM SIGSPATIAL international workshop on spatial semantics and ontologies, in SIGSPATIAL international conference on advances in geographic information system, 19th, Chicago, Ill., 2011, Geographic Information System. 


\section{Workshop Review Form}

Was the content of the workshop relevant and appropriate for the level intended (introductory tutorial on semantics)?

What parts of the workshop were done well?

What parts need improvement? Can you suggest improvements?

Open comments:

Thank you for your comments. 
Publishing support provided by:

Rolla Publishing Service Center

For more information concerning this publication, contact:

Director, USGS Center of Excellence for Geospatial Information

Science (CEGIS)

1400 Independence Road

Rolla, M0 65401

(573) 308-3837

Or visit the CEGIS Web site at:

http://cegis.usgs.gov 

宽 